\title{
Pd/C Catalyzed Carbonylation of Azides in the Presence of Amines.
}

Jin Zhao, Zongyang Li, Shuaihu Yan, Shiyang Xu, Ming-An Wang, Bin Fu, Zhenhua Zhang*

Department of Applied Chemistry, China Agricultural University, Beijing 100193, China

$$
\begin{aligned}
& \text { alkyl- } \mathrm{N}_{3} \\
& \text { benzyl- } \mathrm{N}_{3}+\mathrm{R}^{1}-\stackrel{\mathrm{N}}{\mathrm{N}_{-R^{2}}} \underset{\text { CO balloon }}{\stackrel{\text { cat. Pd/C }}{\longrightarrow}} \\
& \text { aryl }-\mathrm{N}_{3} \\
& \underset{H}{\mathrm{H}} \underset{\mathrm{R}^{\prime}}{\mathrm{N}^{-R^{1}}}+\mathrm{N}_{2} \uparrow \\
& \mathrm{R}=\text { alkyl, benzyl, or aryl, } 47 \text { examples } \\
& \text { up to } 98 \% \text { yield }
\end{aligned}
$$

\section{CONTENTS}

1. Materials and methods. S2

2. Syntheses of azides substrates

3. Typical experimental procedure of $\mathrm{Pd} / \mathrm{C}$ catalyzed carbonylation of azides in the presence of amines. S3

4.Additional mechanism study. S4

5. Spectral data for the ureas. S8

6. References. S23

7. ${ }^{1} \mathrm{H}$ and ${ }^{13} \mathrm{C}$ NMR spectra S24 


\section{Materials and methods.}

Unless stated otherwise, all reactions were performed under a nitrogen atmosphere in a Schlenk reaction flask. Aromatic amines, alkyl bromides, alkyl alcohols and XPhos were obtained from Energy Chemicals. Pd/C (10 wt\%, matrix activated carbon support) was obtained from Sigma-Aldrich. All solvents were distilled under a nitrogen atmosphere prior to use. 1,4-Dioxane and toluene were dried over $\mathrm{Na}$ with benzophenone-ketyl intermediate as indicator. $\mathrm{MeCN}$ and DCE were dried over $\mathrm{CaH}_{2}$. For chromatography, 200-300 mesh silica gel (Qingdao, China) was employed. ${ }^{1} \mathrm{H}$ and ${ }^{13} \mathrm{C}$ NMR spectra were recorded at $400 \mathrm{MHz}$ and $100 \mathrm{MHz}$ with Varian Mercury 400 spectrometer, or $300 \mathrm{MHz}$ and $75 \mathrm{MHz}$ with Bruker ARX 300 at ambient temperature. Chemical shifts are reported in ppm using tetramethylsilane as internal standard when $\mathrm{CDCl}_{3}$ was used as solvent. IR spectra were recorded with a Thermo Electron Corporation Nicolet AVATAR 330 FT-IR spectrometer. HRMS data were obtained on a VG ZAB-HS mass spectrometer, Brucker Apex IV FTMS spectrometer. HPLC (Agilent

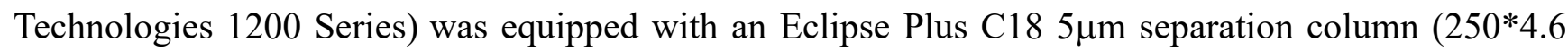
$\mathrm{mm})$. The HRTEM method was used a JEM-2100F. This machine was operated at $200 \mathrm{kV}$, and it was equipped with a field emission gun, ultra-high-resolution pole piece, and ultrathin window JEOL detector. HRTEM images were obtained with an OSIS CANTEGA CCD camera.

\section{Syntheses of azides substrates}

\section{A. Syntheses of aromatic azides substrates}

To a $100 \mathrm{~mL}$ round bottom flask was charged with aniline $(930 \mathrm{mg}, 10 \mathrm{mmol})$ and water $(15 \mathrm{~mL})$. Concentrated $\mathrm{HCl}(4 \mathrm{~mL})$ was added to the vigorously stirred reaction mixture in an ice-water bath. After stirring and cooling to $0{ }^{\circ} \mathrm{C}$ for 20-30 min, a freshly prepared, ice cold solution of $\mathrm{NaNO}_{2}(690$ $\mathrm{mg}, 10 \mathrm{mmol})$ in water $(3 \mathrm{~mL})$ was added dropwise to the reaction mixture while keeping the internal temperature between $0-5{ }^{\circ} \mathrm{C}$. After addition of $\mathrm{NaNO}_{2}$, the reaction mixture was stirred for an additional $10 \mathrm{~min}$. A freshly prepared solution of sodium azide $(780 \mathrm{mg}, 12 \mathrm{mmol})$ in water $(5 \mathrm{~mL})$ was added drop wise to the reaction mixture via additional funnel while maintaining the internal temperature of the reaction mixture below $5{ }^{\circ} \mathrm{C}$. Upon complete addition of the sodium azide solution, the reaction mixture was stirred for an additional $20-30 \mathrm{~min}$ at $0{ }^{\circ} \mathrm{C}$, followed by stirring at $\mathrm{rt}$ for another $3 \mathrm{~h}$. The reaction mixture was extracted with $\mathrm{CH}_{2} \mathrm{Cl}_{2}(2 \times 50 \mathrm{~mL})$, and combined organic layers was dried over anhydrous $\mathrm{Na}_{2} \mathrm{SO}_{4}$, filtered and concentrated under reduced pressure. The crude azidobenzene was further purified by flash column chromatography over a short plug of silica gel using using petroleum ether /EtOAc (15:1) as eluent to afford a yellow liquid (910 mg, 76\%).

\section{B. Syntheses of alkyl azides substrates}

To a stirred solution of benzyl bromide $(171 \mathrm{mg}, 1 \mathrm{mmol})$ in $50 \mathrm{~mL}$ of $1: 1 \mathrm{H}_{2} \mathrm{O} / \mathrm{Me}_{2} \mathrm{CO}(10 \mathrm{~mL})$ was added $\mathrm{NaN}_{3}(195 \mathrm{mg}, 3 \mathrm{mmol})$. After $24 \mathrm{~h}$, the reaction was extracted with EtOAc $(3 \times 20 \mathrm{~mL})$, washed with brine, dried over $\mathrm{MgSO}_{4}$, and concentrated under reduced pressure to give benzene azide as pale yellow oil. The crude product was further purified by flash column chromatography over a short plug of silica gel using petroleum ether /EtOAc (15:1) as eluent to afford a yellow liquid (106 mg, 80\%). 


\section{Typical experimental procedure of $\mathrm{Pd} / \mathrm{C}$-catalyzed carbonylation of azides in the presence of amines.}

\section{A. Typical experimental procedure of $\mathrm{Pd} / \mathrm{C}$ catalyzed carbonylation of benzyl azides in the presence of amines.}

The Schlenk reaction flask was degassed for two times with nitrogen, under a nitrogen atmosphere, $\mathrm{Pd} / \mathrm{C}$ (21 mg, $0.02 \mathrm{mmol}$ ), XPhos (19 mg, $0.04 \mathrm{mmol}$ ) and 4-methoxyaniline (59 mg, $0.48 \mathrm{mmol}$ ) were added. Then the flask was sealed and evacuated to a vacuum of $15 \mathrm{mmHg}$ and fitted a $\mathrm{CO}$ balloon. Benzyl azides $(53 \mathrm{mg}, 0.4 \mathrm{mmol})$ and PhMe $(4 \mathrm{ml})$ were added via syringe. The mixture was stirred at $60{ }^{\circ} \mathrm{C}$ until 1a disappeared as judged by TLC. Solution was removed in vacuo to leave a residue which was purified by flash silica gel chromatography using petroleum ether /EtOAc (from 3:1 to 1:1) as eluent afford pure 3a as a white solid (94 $\mathrm{mg}, 91 \%)$.

B. Typical experimental procedure of $\mathrm{Pd} / \mathrm{C}$ catalyzed carbonylation of alkyl azides in the presence of amines.

The Schlenk reaction flask was degassed for two times with nitrogen, under a nitrogen atmosphere, $\mathrm{Pd} / \mathrm{C}(21 \mathrm{mg}, 0.02 \mathrm{mmol})$ and XPhos $(19 \mathrm{mg}, 0.04 \mathrm{mmol})$ were added. Then the flask was sealed and evacuated to a vacuum of $15 \mathrm{mmHg}$ and fitted a $\mathrm{CO}$ balloon. (3-azidopropyl)benzene (78 $\mathrm{mg}, 0.48$ mmol), and PhMe $(4 \mathrm{ml})$ were added via syringe. The mixture was stirred at $60{ }^{\circ} \mathrm{C}$ for $4 \mathrm{~h}$, until $4 \mathbf{a}$ disappeared as judged by TLC. Then n-butylamine $(30 \mathrm{mg}, 0.4 \mathrm{mmol})$ was added via syringe. The system was stirred at $60{ }^{\circ} \mathrm{C}$ for $8 \mathrm{~h}$. Solution was removed in vacuo to leave a residue which was purified by flash silica gel chromatography using petroleum ether /EtOAc (from 5:1 to 1:1) to afford pure 5a as pale yellow oil (90 mg, 96\%).

Table S1. Reaction Optimization for Pd/C Catalyzed Carbonylation of Alkyl Azides in the Presence of Amines. ${ }^{a}$

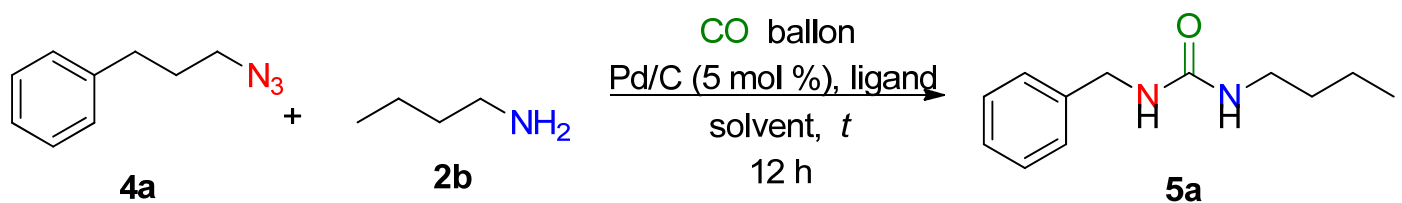

\begin{tabular}{cccccc}
\hline entry & ligand $(\mathrm{mol} \%)$ & solvent & addtive & $t\left({ }^{\circ} \mathrm{C}\right)$ & yield $(\%)^{b}$ \\
\hline 1 & XPhos (10) & dioxane & $/$ & 80 & 15 \\
2 & XPhos (10) & DCE & $/$ & 80 & 10 \\
3 & XPhos (10) & PhMe & $/$ & 80 & 46 \\
4 & DPPP (5) & PhMe & $/$ & 80 & 12 \\
5 & DPPF (5) & PhMe & $/$ & 80 & 11
\end{tabular}




\begin{tabular}{|c|c|c|c|c|c|}
\hline 6 & XPhos (10) & $\mathrm{PhMe}$ & $\mathrm{Et}_{3} \mathrm{~N}$ (1.5 eq.) & 80 & 60 \\
\hline 7 & XPhos (10) & $\mathrm{PhMe}$ & $\mathrm{PhOH}$ (1.5 eq.) & 80 & 50 \\
\hline 8 & XPhos (10) & PhMe & l & 60 & 62 \\
\hline 9 & $\mathrm{P}(\mathrm{t}-\mathrm{Bu})_{3} \mathrm{HBF}_{4}(10)$ & $\mathrm{PhMe}$ & l & 60 & trace \\
\hline 10 & XPhos (10) & $\mathrm{PhMe}$ & $\mathrm{MgO}$ (2 eq.) & 60 & $64^{c}$ \\
\hline 11 & XPhos (20) & $\mathrm{PhMe}$ & 1 & 60 & $61^{d}$ \\
\hline 12 & XPhos (10) & PhMe & $\mathrm{MgO}$ (2 eq.) & 60 & $79^{c, e}$ \\
\hline 13 & XPhos (10) & PhMe & l & 60 & $96^{e, f}$ \\
\hline
\end{tabular}

${ }^{a}$ Reaction was carried out with 1.0 equiv of $\mathbf{4 a}$ and 1.2 equiv of $\mathbf{2 b} .{ }^{b}$ Isolated yield. ${ }^{c} \mathbf{2} \mathbf{b}$ was added after $12 \mathrm{~h} .{ }^{d}$ The reaction was carried out with $10 \mathrm{~mol} \% \mathrm{Pd} / \mathrm{C}$. ${ }^{e}$ The reaction was carried out with 1.2 equiv of $\mathbf{4 a}$ and 1.0 equiv of $\mathbf{2 b} .{ }^{f} \mathbf{2} \mathbf{b}$ was added after $4 \mathrm{~h}$.

\section{Typical experimental procedure of $\mathrm{Pd} / \mathrm{C}$ catalyzed carbonylation of aryl azides in the presence of amines.}

The Schlenk reaction flask was degassed for two times with nitrogen, under a nitrogen atmosphere, $\mathrm{Pd} / \mathrm{C}$ (21 mg, $0.02 \mathrm{mmol}$ ), XPhos (19 mg, $0.04 \mathrm{mmol}$ ) and 4-methoxyaniline (59 mg, $0.48 \mathrm{mmol}$ ) were added. Then the flask was sealed and evacuated to a vacuum of $15 \mathrm{mmHg}$ and fitted a $\mathrm{CO}$ balloon. Azidobenzene (48 mg, $0.4 \mathrm{mmol}$ ) and water (with $5 \% \mathrm{PhMe}$ and $5 \%{ }^{\mathrm{n}} \mathrm{Bu}_{4} \mathrm{NCl}$ ) were added via syringe. The mixture was stirred at room temperature until 6a disappeared as judged by TLC. Solution was removed in vacuo to leave a residue which was purified by flash silica gel chromatography using petroleum ether /EtOAc (from 5:1 to 1:2) to afford pure 7a as a white solid (94 mg, 97\%).

Table S3. Reaction Optimization for Pd/C Catalyzed Carbonylation of Aryl Azides in the Presence of Amines. ${ }^{a}$

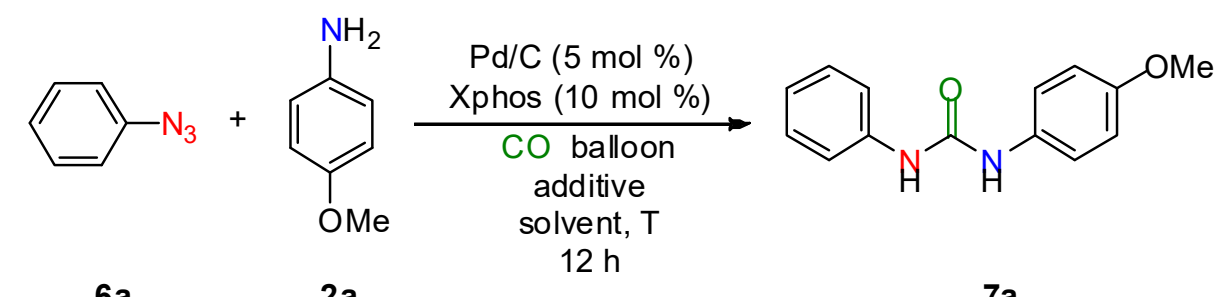

$6 a$

$2 a$

$7 a$

\begin{tabular}{ccccc}
\hline entry & Additive & Solvent & $t\left({ }^{\circ} \mathrm{C}\right)$ & Yield $^{b}$ \\
\hline 1 & $/$ & $\mathrm{PhMe}$ & 25 & $90 \%$ \\
2 & $/$ & $\mathrm{H}_{2} \mathrm{O}^{b}$ & 25 & $65 \%$ \\
$\mathbf{3}$ & $\mathbf{5 \%}$ PTC $\left(\mathbf{n B u} \mathbf{H C l}_{\mathbf{4}} \mathbf{N C}\right.$ & $\mathbf{H}_{\mathbf{2}} \mathbf{O}^{\boldsymbol{c}}$ & $\mathbf{2 5}$ & $\mathbf{9 7 \%}$ \\
\hline
\end{tabular}

${ }^{a}$ Reaction was carried out with 1.0 equiv of $\mathbf{6 a}$ and 1.2 equiv of $\mathbf{2 a} .{ }^{b}$ Isolated yield. ${ }^{c}$ The solvent was added 5\% PhMe. 


\section{Additional mechanism Study}

a) Controlled experiment ${ }^{a}$

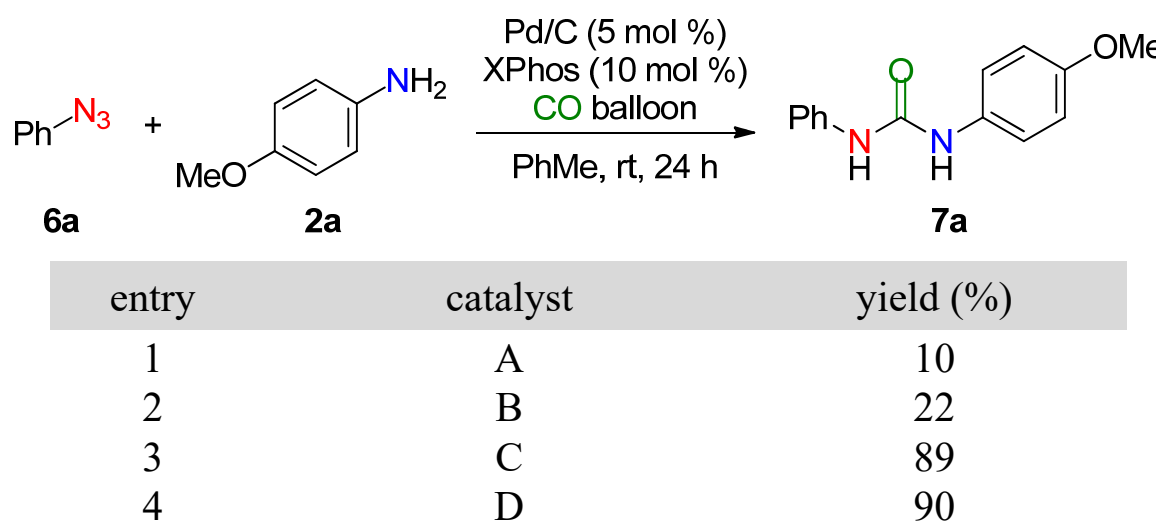

${ }^{a}$ Reaction conditions: 6a $(0.4 \mathrm{mmol}), \mathbf{2 a}(0.48 \mathrm{mmol})$, catalyst and PhMe $(4 \mathrm{ml})$ was added and reacted for $12 \mathrm{~h}$ under $\mathrm{CO}(1 \mathrm{~atm})$.

Catalyst A: $\mathrm{Pd} / \mathrm{C}(0.02 \mathrm{mmol})$ and XPhos $(0.04 \mathrm{mmol})$ were incubated in $\mathrm{PhMe}(4 \mathrm{~mL})$ overnight at $30^{\circ} \mathrm{C}$, then filtrated, and washed with acetone $(3 \times 5 \mathrm{~mL})$, the solution was removed in vacuum to leave a residue as a white solid, which was confirmed mainly to be XPhos. This white solid was used as catalyst A.

Catalyst B: $\mathrm{Pd} / \mathrm{C}(0.02 \mathrm{mmol})$ and XPhos $(0.04 \mathrm{mmol})$ were incubated in $\mathrm{PhMe}(4 \mathrm{~mL})$ overnight at $30^{\circ} \mathrm{C}$, then filtrated, washed with acetone $(3 \times 5 \mathrm{~mL})$, the black residue solid was taken in vacuum to further remove the solvent. This black solid was used as catalyst B.

Catalyst C: Additional fresh XPhos $(0.04 \mathrm{mmol})$ was added to catalyst B.

Catalyst D: Fresh Pd/C (0.02 mmol) and fresh XPhos $(0.04 \mathrm{mmol})$ were used as catalyst D.

\section{b) Recycling test}

The recycling test of $\mathrm{Pd} / \mathrm{C}$ with/without extra XPhos confirmed the consequence. Yields were obtained through HPLC.

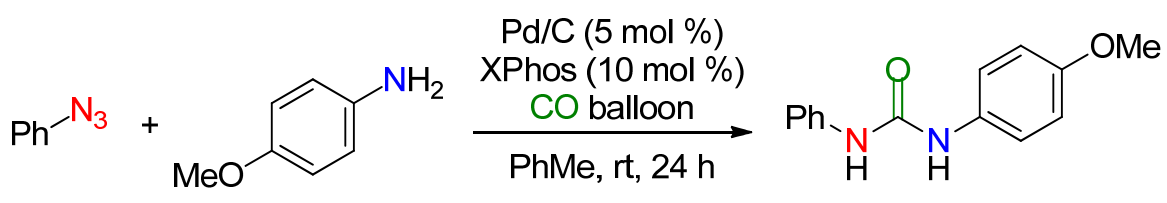

$6 a$

$2 a$

$7 a$

\begin{tabular}{ccc} 
& \multicolumn{2}{c}{ yield $(\%)^{a}$} \\
run & condition $\mathrm{A}^{b}$ & condition $\mathrm{B}^{c}$ \\
1 & 94 & $/$ \\
2 & 88 & 7 \\
3 & 88 & 1 \\
4 & 86 & 0.7 \\
5 & 82 & 0.5
\end{tabular}

${ }^{a}$ Yield was determined by HPLC analysis. ${ }^{b}$ Reaction condition A: 6a $(0.4 \mathrm{mmol}), \mathbf{2 a}(0.48 \mathrm{mmol})$ and PhMe $(4 \mathrm{ml})$ was added and reacted for $12 \mathrm{~h}$ under $\mathrm{CO}(1 \mathrm{~atm})$. Reaction products carried out with the same batch of $\mathrm{Pd} / \mathrm{C}$ with fresh XPhos $(0.04 \mathrm{mmol}) .{ }^{c}$ Reaction condition B: 6a $(0.4 \mathrm{mmol}), \mathbf{2 a}(0.48 \mathrm{mmol})$ and PhMe $(4 \mathrm{ml})$ was added and 
reacted for $12 \mathrm{~h}$ under $\mathrm{CO}(1 \mathrm{~atm})$. Reaction products carried out with the same batch of $\mathrm{Pd} / \mathrm{C}$. Besides, no addition XPhos was added in each run.

Controlled experiments suggested that the palladium that on the carbon was the real catalyst, even some palladium leached into the solvent. In addition, XPhos was necessary for this catalytic reaction, and it may coordinate to the palladium that on the carbon,

\section{c) HRTEM experiment}

The Pd/C-XPhos systems were analyzed by the HRTEM method using a JEM-2100F. This machine was operated at $200 \mathrm{kV}$, and it was equipped with a field emission gun, ultra-high-resolution pole piece, and ultrathin window JEOL detector. HRTEM images were obtained with an OSIS CANTEGA CCD camera.

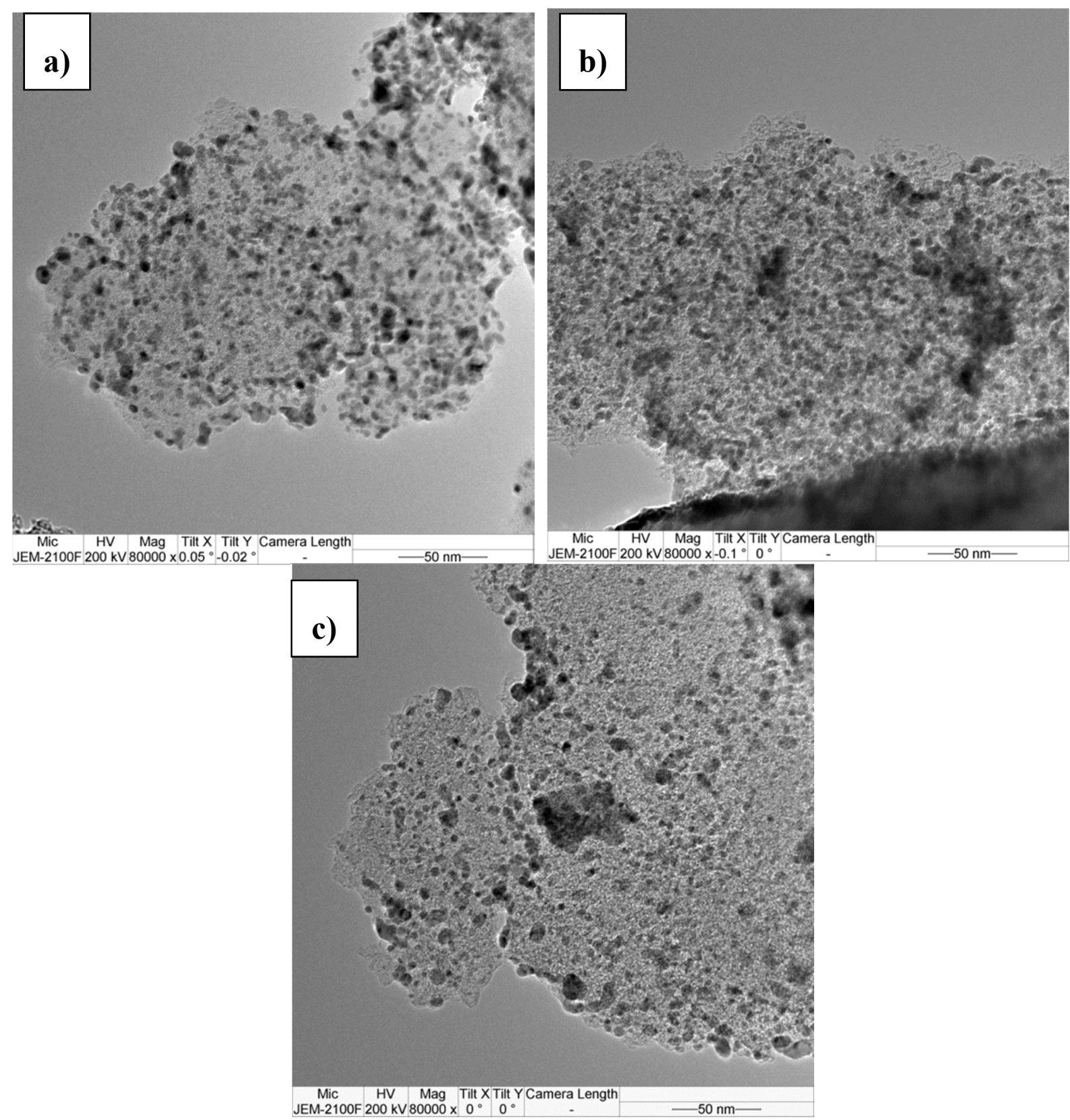

HRTEM of the Pd/C-XPhos systems. a) Fresh Pd/C. b) Pd/C incubated with XPhos in PhMe, then washed with PhMe and acetone. c) Recovered $\mathrm{Pd} / \mathrm{C}$ after one time catalyzed reaction. 
Reflected by HRTEM investigations, all the fresh $\mathrm{Pd} / \mathrm{C}$ (a), filtrated $\mathrm{Pd} / \mathrm{C}$ after incubated with XPhos (b) and recovered $\mathrm{Pd} / \mathrm{C}$ (c), clearly exhibited higher $\mathrm{Pd}$ dispersion and more uniform distribution, no agglomeration of Pd crystallites was observed. This result is different with other heterogeneous catalyzed coupling reaction. (See reference: Heidenreich, R.-G.; Krauter, J. G. E.; Pietsch, J.; Köheler, K. J. Mol. Catal. A: Chem. 2002, 182-183, 499.)

d) Stoichiometric reaction and sub step reaction
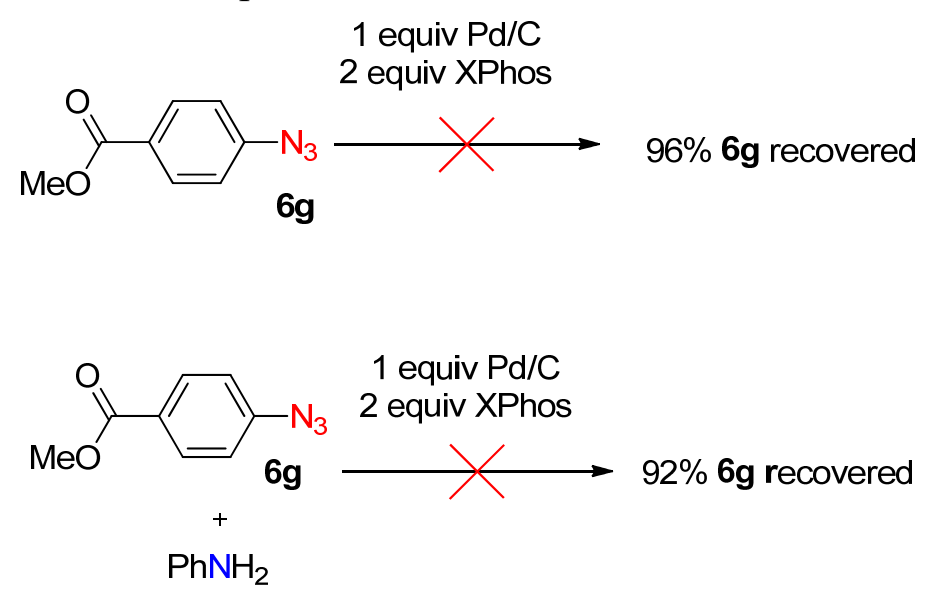

Reaction was carried out with 1.0 equiv of $\mathbf{1 a}(0.4 \mathrm{mmol})$ and 1.2 equiv of $\mathbf{2 a}(0.48 \mathrm{mmol})$.

Above results showed that the coordination of $\mathrm{CO}$ to the palladium catalyst was also critical to the decomposing of azides. When CO was removed, $>90 \%$ azides could be recovered after $24 \mathrm{~h}$ even using the stoichiometric of $\mathrm{Pd} / \mathrm{C}$.

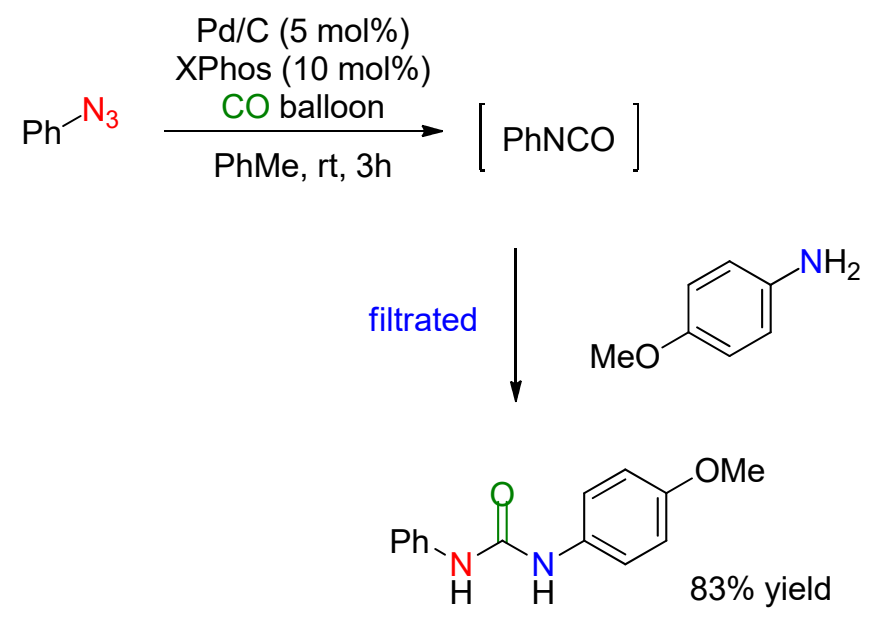




\section{Spectral data for the ureas}

1-benzyl-3-(4-methoxyphenyl)urea (3a) ${ }^{1}$

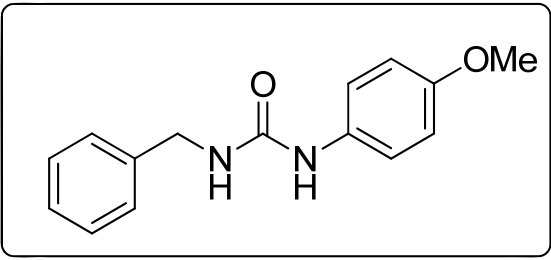

Yellow solid. (94mg, 91\%)

${ }^{1} \mathrm{H}$ NMR (400 MHz, DMSO-d $\left.{ }^{6}\right) \delta 8.35(\mathrm{~s}, 1 \mathrm{H}), 7.38-7.19(\mathrm{~m}, 7 \mathrm{H}), 6.82(\mathrm{~d}, J=8.9 \mathrm{~Hz}, 2 \mathrm{H}), 6.51(\mathrm{t}, J=$ $5.8 \mathrm{~Hz}, 1 \mathrm{H}), 4.29$ (d, $J=5.8 \mathrm{~Hz}, 2 \mathrm{H}), 3.69(\mathrm{~s}, 3 \mathrm{H})$.

${ }^{13} \mathrm{C}$ NMR (100 MHz, DMSO-d $\left.{ }^{6}\right) \delta 155.48,153.97,140.53,133.60,128.31,127.12,126.70,119.48$, $113.89,55.14,42.79$.

1-benzyl-3-butylurea (3b) ${ }^{2}$

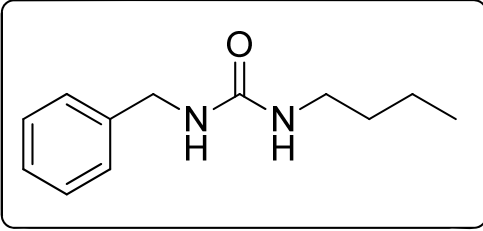

White solid. (81mg, 98\%)

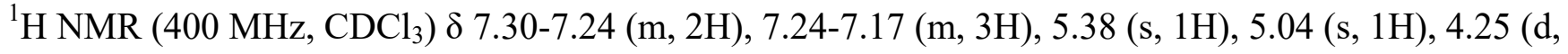
$J=5.8 \mathrm{~Hz}, 2 \mathrm{H}), 3.06(\mathrm{~d}, J=6.2 \mathrm{~Hz}, 2 \mathrm{H}), 1.37(\mathrm{dd}, J=14.9,7.2 \mathrm{~Hz}, 2 \mathrm{H}), 1.26(\mathrm{dd}, J=14.9,7.2 \mathrm{~Hz}$, $2 \mathrm{H}), 0.86(\mathrm{t}, J=7.2 \mathrm{~Hz}, 3 \mathrm{H})$.

${ }^{13} \mathrm{C}$ NMR $\left(100 \mathrm{MHz}, \mathrm{CDCl}_{3}\right) \delta 158.85,139.63,128.61,127.38,127.19,44.35,40.25,32.42,2$ $0.12,13.92$.

1-butyl-3-(4-methylbenzyl)urea (3c) ${ }^{3}$

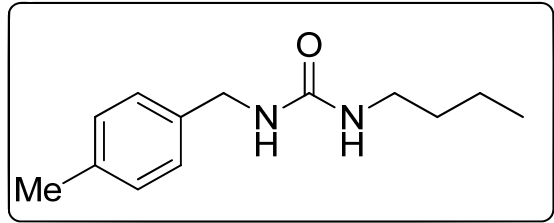

White solid. (68mg, 77\%)

${ }^{1} \mathrm{H}$ NMR $\left(400 \mathrm{MHz}, \mathrm{CDCl}_{3}\right) \delta$ 7.07-7.02 (m, 4H), $5.61(\mathrm{~s}, 1 \mathrm{H}), 5.31(\mathrm{~s}, 1 \mathrm{H}), 4.18-4.14(\mathrm{~m}, 2$ $\mathrm{H}), 3.03-2.98(\mathrm{~m}, 2 \mathrm{H}), 2.27(\mathrm{~s}, 3 \mathrm{H}), 1.36-1.19(\mathrm{~m}, 4 \mathrm{H}), 0.84(\mathrm{t}, J=7.2 \mathrm{~Hz}, 3 \mathrm{H})$.

${ }^{13} \mathrm{C}$ NMR $\left(100 \mathrm{MHz}, \mathrm{CDCl}_{3}\right) \delta 159.09,136.65,136.57,129.18,127.31,43.98,40.10,32.43,21$. $12,20.10,13.89$.

1-butyl-3-(4-methoxybenzyl)urea (3d) ${ }^{4}$

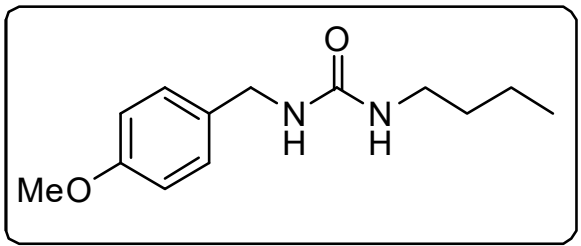


White solid. (69mg, 73\%)

${ }^{1} \mathrm{HNMR}\left(400 \mathrm{MHz}\right.$, DMSO-d $\left.{ }^{6}\right) \delta$ 7.19-7.12 (m, 2H), 6.89-6.84 (m, 2H), $6.15(\mathrm{t}, J=6.0 \mathrm{~Hz}, 1$ $\mathrm{H}), 5.83(\mathrm{t}, J=5.6 \mathrm{~Hz}, 1 \mathrm{H}), 4.11(\mathrm{~d}, J=6.0 \mathrm{~Hz}, 2 \mathrm{H}), 3.72(\mathrm{~s}, 3 \mathrm{H}), 3.01-2.96(\mathrm{~m}, 2 \mathrm{H}), 1.3$ 6-1.29 (m, 2H), 1.29-1.22 (m, 2H), $0.86(\mathrm{t}, J=7.2 \mathrm{~Hz}, 3 \mathrm{H})$.

${ }^{13} \mathrm{C}$ NMR (100MHz, DMSO-d $\left.{ }^{6}\right) \delta 158.02,158.00,132.90,128.30,113.59,55.04,42.35,38.96,32.18$, $19.53,13.73$.

1-butyl-3-(4-chlorobenzyl)urea (3e) ${ }^{5}$

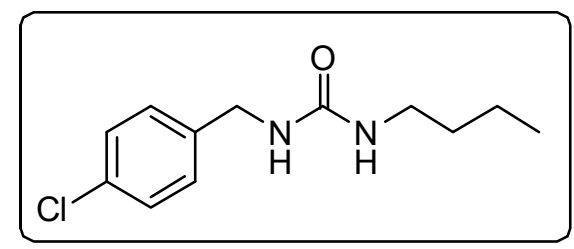

White solid. (68mg, 71\%)

${ }^{1} \mathrm{H}$ NMR (400 MHz, DMSO-d $\left.{ }^{6}\right) \delta 7.36(\mathrm{dd}, J=8.3,1.9 \mathrm{~Hz}, 2 \mathrm{H}), 7.25(\mathrm{~d}, J=8.3 \mathrm{~Hz}, 2 \mathrm{H})$, $6.31(\mathrm{t}, J=5.9 \mathrm{~Hz}, 1 \mathrm{H}), 5.94(\mathrm{t}, J=5.6 \mathrm{~Hz}, 1 \mathrm{H}), 4.17(\mathrm{~d}, J=6.1 \mathrm{~Hz}, 2 \mathrm{H}), 3.01-2.97(\mathrm{~m}$, $2 \mathrm{H}), 1.39-1.31(\mathrm{~m}, 2 \mathrm{H}), 1.26(\mathrm{dd}, J=15.0,7.2 \mathrm{~Hz}, 2 \mathrm{H}), 0.86(\mathrm{t}, J=7.2 \mathrm{~Hz}, 3 \mathrm{H})$.

${ }^{13} \mathrm{C}$ NMR (100 MHz, DMSO-d $\left.{ }^{6}\right) \delta$ 158.04, 140.26, 130.98, 128.82, 128.11, 42.21, 39.03, 32.17, $19.56,13.75$.

\section{1-butyl-3-(4-fluorobenzyl)urea (3f)}

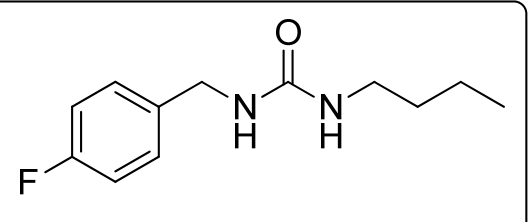

White solid. (80mg, 89\%)

${ }^{1} \mathrm{H}$ NMR (400 MHz, $\left.\mathrm{CDCl}_{3}\right) \delta$ 7.20-7.14 (m, 2H), 7.00- $6.89(\mathrm{~m}, 2 \mathrm{H}), 5.21(\mathrm{~s}, 1 \mathrm{H}), 4.87(\mathrm{~s}, 1 \mathrm{H})$, 4.25-4.21 (m, 2H), 3.08-3.05 (m, 2H), 1.42-1.32 (m, 2H), 1.31- $1.22(\mathrm{~m}, 2 \mathrm{H}), 0.87(\mathrm{t}, J=7.2 \mathrm{~Hz}, 3 \mathrm{H})$. ${ }^{13} \mathrm{C}$ NMR $\left(100 \mathrm{MHz}, \mathrm{CDCl}_{3}\right) \delta 162.09(\mathrm{~d}, J=242.0 \mathrm{~Hz}), 158.64,135.40,129.01(\mathrm{~d}, J=8.2 \mathrm{~Hz})$, 115.44 (d, $J=21.4 \mathrm{~Hz}), 43.67,40.33,32.41,20.13,13.89$.

${ }^{19} \mathrm{~F}$ NMR $\left(376 \mathrm{MHz}, \mathrm{CDCl}_{3}\right) \delta-115.6$.

IR (neat) v: 2959, 2929, 1624, 1587, 1511, 1228, 1157, $825 \mathrm{~cm}^{-1}$.

HRMS (ESI) calcd for $\mathrm{C}_{12} \mathrm{H}_{18} \mathrm{FN}_{2} \mathrm{O}: 225.13977$, found: 225.13973

1-butyl-3-(3-fluorobenzyl)urea (3g)

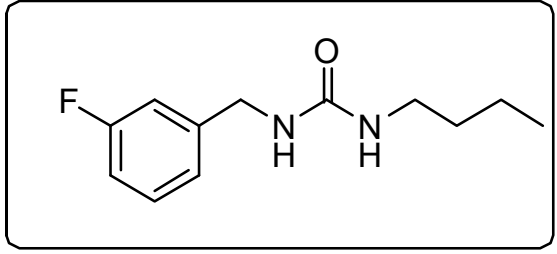

White solid. (91mg, >98\%) 
${ }^{1} \mathrm{H}$ NMR (400 MHz, $\left.\mathrm{CDCl}_{3}\right)$ d 7.26-7.22 (m, 1H), 7.01 (d, J=7.6 Hz, 1H), 6.97-6.89 (m, 2H), 5.20 (s, $1 \mathrm{H}), 4.82(\mathrm{~s}, 1 \mathrm{H}), 4.31$ (d, $J=5.8 \mathrm{~Hz}, 2 \mathrm{H}), 3.15-3.10(\mathrm{~m}, 2 \mathrm{H}), 1.48-1.37$ (m, 2H), 1.34-1.25 (m, 2H), $0.89(\mathrm{t}, J=7.3 \mathrm{~Hz}, 3 \mathrm{H})$.

${ }^{13} \mathrm{C}$ NMR $\left(100 \mathrm{MHz}, \mathrm{CDCl}_{3}\right) \delta 162.90(\mathrm{~d}, J=244.3 \mathrm{~Hz}), 159.21,142.64(\mathrm{~d}, J=6.8 \mathrm{~Hz}), 1$ $29.80(\mathrm{~d}, J=8.2 \mathrm{~Hz}), 122.28(\mathrm{~d}, J=2.6 \mathrm{~Hz}), 113.71(\mathrm{~d}, J=3.0 \mathrm{~Hz}), 113.50(\mathrm{~d}, J=3.4 \mathrm{H}$ z), 43.25, 39.94, 32.29, 19.97, 13.72 .

${ }^{19} \mathrm{~F}$ NMR $\left(376 \mathrm{MHz}, \mathrm{CDCl}_{3}\right) \delta-113.1(\mathrm{dd}, J=15.2,9.0 \mathrm{~Hz})$.

IR (neat) v: 2957, 2934, 1626, 1585, 1256, 1065, $815 \mathrm{~cm}^{-1}$.

HRMS (ESI) calcd for $\mathrm{C}_{12} \mathrm{H}_{18} \mathrm{FN}_{2} \mathrm{O}$ : 225.13977, found: 225.14041 .

1-butyl-3-(4-(trifluoromethyl)benzyl)urea (3h)

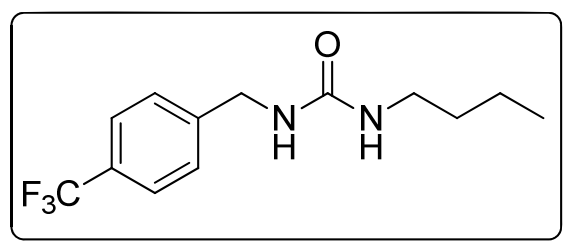

White solid. (109mg, $>98 \%$ )

${ }^{1} \mathrm{H}$ NMR $\left(400 \mathrm{MHz}, \mathrm{CDCl}_{3}\right) \delta 7.49(\mathrm{~d}, J=8.0 \mathrm{~Hz}, 2 \mathrm{H}), 7.33-7.31(\mathrm{~m}, 2 \mathrm{H}), 5.70(\mathrm{~s}, 1 \mathrm{H}), 5.24(\mathrm{~s}, 1 \mathrm{H})$, $4.28(\mathrm{~d}, J=6.0 \mathrm{~Hz}, 2 \mathrm{H}), 3.09-3.04(\mathrm{~m}, 2 \mathrm{H}), 1.41-1.33(\mathrm{~m}, 2 \mathrm{H}), 1.28-1.23(\mathrm{~m}, 2 \mathrm{H}), 0.86(\mathrm{t}, J=7.3 \mathrm{~Hz}$, $3 \mathrm{H})$.

${ }^{13} \mathrm{C}$ NMR $\left(100 \mathrm{MHz} \mathrm{CDCl}_{3}\right) \delta 158.56,143.89,129.55(\mathrm{q}, J=32.2 \mathrm{~Hz}), 127.46,125.58(\mathrm{q}, J=3.8$ $\mathrm{Hz}), 124.21$ (q, $J=263.8 \mathrm{~Hz}), 43.86,40.41,32.40,20.12,13.86$.

${ }^{19} \mathrm{~F} \mathrm{NMR}\left(376 \mathrm{MHz}, \mathrm{CDCl}_{3}\right) \delta-62.5$.

IR (neat) v: 3334, 2964, 2930, 1629, 1581, 1329, $1125 \mathrm{~cm}^{-1}$.

HRMS (ESI) calcd for $\mathrm{C}_{13} \mathrm{H}_{18} \mathrm{~F}_{3} \mathrm{~N}_{2} \mathrm{O}: 275.13657$, found: 275.13731 .

1-butyl-3-(2-(trifluoromethyl)benzyl)urea (3i)<smiles>CCCCNC(=O)NCc1ccccc1C(F)(F)F</smiles>

White solid. (95mg, 87\%)

${ }^{1} \mathrm{HNMR}\left(400 \mathrm{MHz}, \mathrm{CDCl}_{3}\right) \delta$ 7.63-7.60 (m, 2H), 7.53-7.49 (m, 1H), 7.37-7.33 (m, 1H), 4.80 $(\mathrm{s}, 1 \mathrm{H}), 4.55(\mathrm{~d}, J=6.1 \mathrm{~Hz}, 2 \mathrm{H}), 4.43(\mathrm{~s}, 1 \mathrm{H}), 3.17-3.12(\mathrm{~m}, 2 \mathrm{H}), 1.48-1.41(\mathrm{~m}, 2 \mathrm{H}), 1.33-1$. $27(\mathrm{~m}, 2 \mathrm{H}), 0.90(\mathrm{t}, J=7.3 \mathrm{~Hz}, 3 \mathrm{H})$.

${ }^{13} \mathrm{C} \mathrm{NMR}\left(100 \mathrm{MHz}, \mathrm{CDCl}_{3}\right) \delta 158.05,138.14,132.43,132.42,130.46,127.44,126.03(\mathrm{q}, J=5.7 \mathrm{~Hz})$, $124.74(\mathrm{q}, J=288.3 \mathrm{~Hz}), 41.03,40.54,32.36,20.12,13.88$.

${ }^{19} \mathrm{~F}$ NMR $\left(376 \mathrm{MHz}, \mathrm{CDCl}_{3}\right) \delta-59.6$.

IR (neat) $v: 1630,1594,1317,1271,1160,1117,690 \mathrm{~cm}^{-1}$.

HRMS (ESI) calcd for $\mathrm{C}_{13} \mathrm{H}_{18} \mathrm{~F}_{3} \mathrm{~N}_{2} \mathrm{O}: 275.13657$, found: 275.13714 . 
<smiles>CCCCNC(=O)NCc1cccc(C(F)(F)F)c1</smiles>

White solid. (98mg, 90\%)

${ }^{1} \mathrm{H}$ NMR $\left(400 \mathrm{MHz}, \mathrm{CDCl}_{3}\right) \delta$ 7.53-7.35 (m, 4H), $5.11(\mathrm{~s}, 1 \mathrm{H}), 4.71(\mathrm{~s}, 1 \mathrm{H}), 4.38(\mathrm{~d}, J=6.0 \mathrm{~Hz}, 2 \mathrm{H})$, 3.16-3.11 (m, 2H), 1.47-1.40 (m, 2H), 1.33-1.26 (m, 2H), 0.89 (t, J=7.3 Hz, 3H).

${ }^{13} \mathrm{C}$ NMR $\left(100 \mathrm{MHz}, \mathrm{CDCl}_{3}\right) \delta 158.39,140.80,130.84,130.82,129.15,125.40$ (q, $\left.J=269.8 \mathrm{~Hz}\right)$, $124.14(\mathrm{q}, J=3.8 \mathrm{~Hz}), 123.99(\mathrm{q}, J=3.8 \mathrm{~Hz}), 43.96,40.45,32.39,20.12,13.87$.

${ }^{19} \mathrm{~F} \mathrm{NMR}\left(376 \mathrm{MHz}, \mathrm{CDCl}_{3}\right) \delta-62.7$.

IR (neat) $v: 1636,1580,1331,1166,1129,703,689 \mathrm{~cm}^{-1}$.

HRMS (ESI) calcd for $\mathrm{C}_{13} \mathrm{H}_{18} \mathrm{~F}_{3} \mathrm{~N}_{2} \mathrm{O}: 275.13657$, found: 275.13710 .

1-butyl-3-(naphthalen-2-ylmethyl)urea (3k)<smiles>CCCCNC(=O)NCc1ccc2ccccc2c1</smiles>

White solid. (64mg, 63\%)

${ }^{1} \mathrm{H}$ NMR $\left(400 \mathrm{MHz}, \mathrm{CDCl}_{3}\right) \delta$ 7.99-7.93 (m, 1H), 7.82-7.78 (m, 1H), 7.73-7.71 (M, 1H), 7.50-7.42 (m, 2H), 7.35-7.31 (M, 2H), $5.01(\mathrm{~s}, 1 \mathrm{H}), 4.73(\mathrm{~s}, 1 \mathrm{H}), 4.67(\mathrm{~s}, 2 \mathrm{H}), 3.04(\mathrm{t}, J=7.0 \mathrm{~Hz}, 2 \mathrm{H}), 1.40-1.30(\mathrm{~m}$, 2H), $1.28-1.20(\mathrm{~m}, 2 \mathrm{H}), 0.84(\mathrm{t}, J=7.3 \mathrm{~Hz}, 3 \mathrm{H})$.

${ }^{13} \mathrm{C}$ NMR $\left(100 \mathrm{MHz}, \mathrm{CDCl}_{3}\right) \delta 158.69,134.73,133.78,131.35,128.64,128.03,126.29,125.7$ $8,125.57,125.39,123.52,42.24,40.13,32.37,20.05,13.85$.

IR (neat) $v: 2959,2928,1626,1581,1252,106,775 \mathrm{~cm}^{-1}$.

HRMS (ESI) calcd for $\mathrm{C}_{16} \mathrm{H}_{21} \mathrm{~N}_{2} \mathrm{O}: 257.16484$, found: 257.16519 .

1-butyl-3-(thiophen-2-ylmethyl)urea (31)

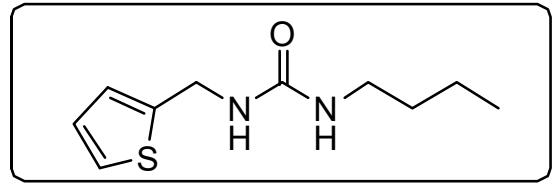

White solid. (70mg, 85\%)

${ }^{1} \mathrm{H}$ NMR $\left(400 \mathrm{MHz}, \mathrm{CDCl}_{3}\right) \delta 7.21(\mathrm{ddd}, J=4.9,1.5,0.5 \mathrm{~Hz}, 1 \mathrm{H}), 6.97-6.92(\mathrm{~m}, 2 \mathrm{H}), 4.71$ $(\mathrm{s}, 1 \mathrm{H}), 4.54(\mathrm{~d}, J=5.7 \mathrm{~Hz}, 2 \mathrm{H}), 4.39(\mathrm{~s}, 1 \mathrm{H}), 3.19-3.14(\mathrm{~m}, 2 \mathrm{H}), 1.50-1.43(\mathrm{~m}, 2 \mathrm{H}), 1.37-$ $1.30(\mathrm{~m}, 2 \mathrm{H}), 0.91(\mathrm{t}, J=7.3 \mathrm{~Hz}, 3 \mathrm{H})$.

${ }^{13} \mathrm{C}$ NMR $\left(100 \mathrm{MHz}, \mathrm{CDCl}_{3}\right) \delta 157.69,142.63,126.97,125.52,125.08,40.54,39.64,32.38,2$ $0.14,13.92$.

IR (neat) v: 3338, 2955, 1627, 1589, $689 \mathrm{~cm}^{-1}$.

HRMS (ESI) calcd for $\mathrm{C}_{10} \mathrm{H}_{17} \mathrm{~N}_{2} \mathrm{OS}$ : 213.10561, found: 213.10571 . 
$N$-benzylpiperidine-1-carboxamide $(\mathbf{3 m})^{6}$

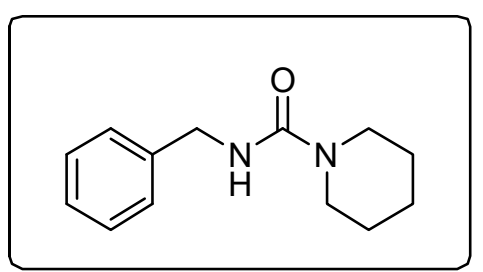

White solid. (74mg, 85\%)

${ }^{1} \mathrm{H}$ NMR (400 MHz, $\left.\mathrm{CDCl}_{3}\right) \delta 7.35-7.23(\mathrm{~m}, 5 \mathrm{H}), 4.77(\mathrm{~s}, 1 \mathrm{H}), 4.42(\mathrm{~d}, J=5.5 \mathrm{~Hz}, 2 \mathrm{H}), 3$. 36-3.31 (m, 4H), 1.62-1.52 (m, 6H).

${ }^{13} \mathrm{C}$ NMR $\left(100 \mathrm{MHz}, \mathrm{CDCl}_{3}\right) \delta 157.69,139.83,128.67,127.86,127.30,45.12,45.07,25.74,24.53$.

1-benzyl-3-phenylurea $(\mathbf{3 n})^{7}$

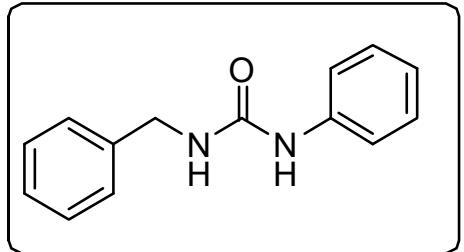

White solid. (88mg, 97\%)

${ }^{1} \mathrm{H}$ NMR (400 MHz, DMSO-d $\left.{ }^{6}\right) \delta 8.55(\mathrm{~s}, 1 \mathrm{H}), 7.40(\mathrm{~d}, J=7.7 \mathrm{~Hz}, 2 \mathrm{H}), 7.36-7.28(\mathrm{~m}, 4 \mathrm{H})$, 7.26-7.20 (m, 3H), 6.91-6.87 (m, 1H), $6.61(\mathrm{t}, J=5.9 \mathrm{~Hz}, 1 \mathrm{H}), 4.30(\mathrm{~d}, J=5.9 \mathrm{~Hz}, 2 \mathrm{H})$.

${ }^{13} \mathrm{C}$ NMR (100 MHz, DMSO-d $\left.{ }^{6}\right) \delta 155.25,140.48,140.38,128.69,128.34,127.14,126.75,12$ $1.12,117.70,42.75$.

1-benzyl-3-mesitylurea (3o)<smiles>Cc1cc(C)c(NC(=O)NCc2ccccc2)c(C)c1</smiles>

White solid. (100mg, 93\%)

${ }^{1} \mathrm{H}$ NMR (400 MHz, DMSO-d $\left.\mathrm{d}^{6}\right) \delta 7.44(\mathrm{~s}, 1 \mathrm{H}), 7.34-7.22(\mathrm{~m}, 5 \mathrm{H}), 6.84(\mathrm{~s}, 2 \mathrm{H}), 6.43(\mathrm{~s}, 1 \mathrm{H})$, 4.26-4.23 (m, 2H), $2.20(\mathrm{~s}, 3 \mathrm{H}), 2.12(\mathrm{~s}, 6 \mathrm{H})$.

${ }^{13} \mathrm{C}$ NMR (100 MHz, DMSO-d $\left.{ }^{6}\right) \delta 156.29,141.04,135.47,133.32,128.30,128.21,127.00,12$ 6.86, 126.55, 42.95, 20.51, 18.20.

IR (neat) v: 3314, 3290, 1630, 1609, 1566, 1229, $667 \mathrm{~cm}^{-1}$.

HRMS (ESI) calcd for $\mathrm{C}_{17} \mathrm{H}_{21} \mathrm{~N}_{2} \mathrm{O}: 269.16484$, found: 269.16530 . 
benzyl-3-(4-(trifluoromethyl)phenyl)urea (3p)<smiles>O=C(NCc1ccccc1)Nc1ccc(C(F)(F)F)cc1</smiles>

White solid. (95mg, 81\%)

${ }^{1} \mathrm{H}$ NMR (400 MHz, DMSO-d $\left.{ }^{6}\right) \delta 9.02(\mathrm{~s}, 1 \mathrm{H}), 7.62-7.55(\mathrm{~m}, 4 \mathrm{H})$, 7.38-7.29 (m, 4H), 7.28-7.16 (m, $1 \mathrm{H}), 6.79(\mathrm{t}, J=5.9 \mathrm{~Hz}, 1 \mathrm{H}), 4.31(\mathrm{~d}, J=5.9 \mathrm{~Hz}, 2 \mathrm{H})$.

${ }^{13} \mathrm{C}-\mathrm{NMR}\left(100 \mathrm{MHz}, \mathrm{DMSO}-\mathrm{d}^{6}\right) \delta 154.87,144.17,140.04,128.30,127.14,126.77,125.94(\mathrm{q}, J=3.9$ $\mathrm{Hz}), 124.64$ (q, $J=269.1 \mathrm{~Hz}), 121.04$ (q, $J=31.7 \mathrm{~Hz}), 117.29,42.76$.

${ }^{19} \mathrm{~F}$ NMR (376 MHz, DMSO-d $\left.{ }^{6}\right) \delta-61.4$. IR (neat) v: 2130, 1640, 1612, 1566, 1324, $1120 \mathrm{~cm}^{-1}$.

HRMS (ESI) calcd for $\mathrm{C}_{15} \mathrm{H}_{14} \mathrm{~F}_{3} \mathrm{~N}_{2} \mathrm{O}$ : 295.10527, found: 295.10610 .

1-butyl-3-(3-phenylpropyl)urea (5a)<smiles>CCCCNC(=O)NCCCc1ccccc1</smiles>

White solid. (90mg, 96\%)

${ }^{1} \mathrm{H}$ NMR (400 MHz, $\left.\mathrm{CDCl}_{3}\right) \delta$ 7.27-7.25 (m, 2H), 7.18-7.13 (m, 3H), $5.11(\mathrm{~s}, 1 \mathrm{H}), 5.02(\mathrm{~s}, 1 \mathrm{H})$, 3.20-3.15 (m, 2H), 3.13-3.08 (m, 2H), $2.62(\mathrm{~m}, 2 \mathrm{H}), 1.83-1.75(\mathrm{~m}, 2 \mathrm{H}), 1.45-1.39(\mathrm{~m}, 2 \mathrm{H}), 1.35-1.28$ (m, 2H), 0.89 (m, 3H).

${ }^{13} \mathrm{C} \mathrm{NMR}\left(100 \mathrm{MHz}, \mathrm{CDCl}_{3}\right) \delta 159.00,141.78,128.48,128.43,125.96,40.22,40.06,33.32,32.51$, $32.08,20.16,13.92$.

IR (neat) v: 3333, 2930, 2861, 1630,1573, $1259 \mathrm{~cm}^{-1}$.

HRMS (ESI) calcd for $\mathrm{C}_{14} \mathrm{H}_{23} \mathrm{~N}_{2} \mathrm{O}: 235.18049$, found: 235.18107 .

$N$-(3-phenylpropyl)morpholine-4-carboxamide (5b)<smiles>O=C(NCCCc1ccccc1)N1CCOCC1</smiles>

White solid. (94mg, 95\%)

${ }^{1} \mathrm{H}$ NMR (400 MHz, $\mathrm{CDCl}_{3}$ ) $\delta$ 7.29-7.26 (m, 2H), 7.19-7.15 (m, 3H), $4.70(\mathrm{~s}, 1 \mathrm{H}), 3.62-3.59(\mathrm{~m}, 4 \mathrm{H})$, 3.27-3.20 (m, 6H), 2.67-2.63 (m, 2H), 1.88-1.81 (m, 2H).

${ }^{13} \mathrm{C}$ NMR $\left(100 \mathrm{MHz}, \mathrm{CDCl}_{3}\right) \delta 157.82,141.81,128.45,128.35,125.90,66.45,43.85,40.76,33.58$, 31.56 .

IR (neat) v: 2923, 2856, 1712, 1626.1, 1538, 1262, $851 \mathrm{~cm}^{-1}$. 
HRMS (ESI) calcd for $\mathrm{C}_{14} \mathrm{H}_{21} \mathrm{~N}_{2} \mathrm{O}_{2}: 249.15975$, found: 249.16045 .

1,3-bis(3-phenylpropyl)urea (5c)

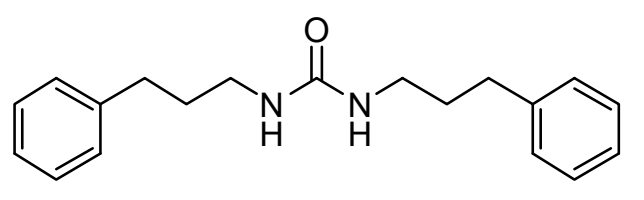

White solid. (92mg, 78\%)

${ }^{1} \mathrm{H}$ NMR $\left(400 \mathrm{MHz}, \mathrm{CDCl}_{3}\right) \delta$ 7.27-7.24 (m, 5H), 7.18-7.14 (m, 5H), $4.36(\mathrm{~s}, 2 \mathrm{H}), 3.17-3.12(\mathrm{~m}, 4 \mathrm{H})$, $2.63(\mathrm{~m}, 4 \mathrm{H}), 1.83-1.75(\mathrm{~m}, 4 \mathrm{H})$.

${ }^{13} \mathrm{C} \mathrm{NMR}\left(100 \mathrm{MHz}, \mathrm{CDCl}_{3}\right) \delta 158.17,141.53,128.41,128.33,125.92,40.11,33.18,31.76$.

IR (neat) v: 2925, 1743, 1633, 1574, 1454, $1259 \mathrm{~cm}^{-1}$.

HRMS (ESI) calcd for $\mathrm{C}_{19} \mathrm{H}_{25} \mathrm{~N}_{2} \mathrm{O} 297.19614$, found: 297.19526.

1-benzyl-3-(3-phenylpropyl)urea (5d)

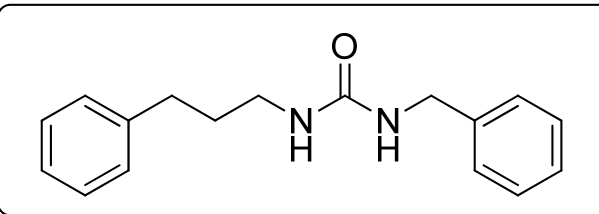

White solid. (83mg, 77\%)

${ }^{1} \mathrm{H}$ NMR $\left(300 \mathrm{MHz}, \mathrm{CDCl}_{3}\right) \delta$ 7.37-7.02 (m, 10H), $5.46(\mathrm{~s}, 1 \mathrm{H}), 5.18(\mathrm{~s}, 1 \mathrm{H}), 4.22(\mathrm{~d}, J=5.7 \mathrm{~Hz}, 2 \mathrm{H})$, 3.10-3.04 (m, 2H), $2.52(\mathrm{t}, J=7.7 \mathrm{~Hz}, 2 \mathrm{H}), 1.78-1.58(\mathrm{~m}, 2 \mathrm{H})$.

${ }^{13} \mathrm{C} \mathrm{NMR}\left(75 \mathrm{MHz}, \mathrm{CDCl}_{3}\right) \delta 158.8,141.7,139.6,128.6,128.5,128.4,127.4,121.2,126.0,44.4,40.1$, $33.2,31.9$.

IR (neat) v: 2957, 2929, 1462, 1380, 1163, 1046, $882 \mathrm{~cm}^{-1}$.

HRMS (ESI) calcd for: $\mathrm{C}_{17} \mathrm{H}_{21} \mathrm{~N}_{2} \mathrm{O}, 269.16484$, found : 269.16528

1-phenyl-3-(3-phenylpropyl)urea $(\mathbf{5 e})^{7}$

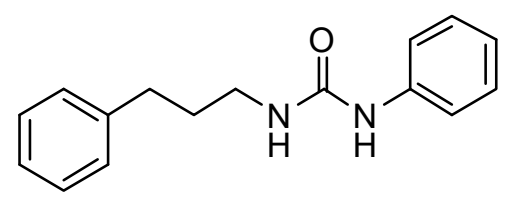

White solid. (96mg, 95\%)

${ }^{1} \mathrm{H}$ NMR (400 MHz, DMSO-d $\left.{ }^{6}\right) \delta 8.41(\mathrm{~s}, 1 \mathrm{H}), 7.41-7.38(\mathrm{~m}, 2 \mathrm{H})$, 7.28-7.26 (m,2H), 7.23-7.19 (m, 5H), 6.90-6.86 (m,1H), $6.20(\mathrm{t}, J=5.5 \mathrm{~Hz}, 1 \mathrm{H}), 3.12-3.08(\mathrm{~m}, 2 \mathrm{H}), 2.62-2.59(\mathrm{~m}, 2 \mathrm{H}), 1.77-1.70(\mathrm{~m}, 2 \mathrm{H})$. ${ }^{13} \mathrm{C}$ NMR (100 MHz, DMSO-d $\left.{ }^{6}\right) \delta 155.23,141.70,140.57,128.60,128.29,128.27,125.72,120.91$, $177.99,38.66,32.50,31.59$. 


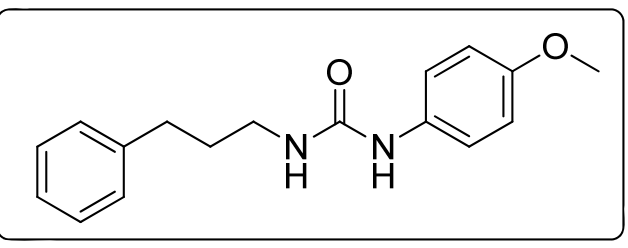

Yellow solid. (91mg, 77\%)

${ }^{1} \mathrm{H}$ NMR (400 MHz, DMSO-d $\left.{ }^{6}\right) \delta 8.20(\mathrm{~s}, 1 \mathrm{H}), 7.34-7.24(\mathrm{~m}, 4 \mathrm{H}), 7.22-7.16(\mathrm{~m}, 3 \mathrm{H}), 6.80(\mathrm{~d}, J=8.6$ $\mathrm{Hz}, 2 \mathrm{H}), 6.08(\mathrm{t}, J=5.6 \mathrm{~Hz}, 1 \mathrm{H}), 3.68(\mathrm{~s}, 3 \mathrm{H}), 3.10-3.05(\mathrm{~m}, 2 \mathrm{H}), 2.59$ (t, $J=7.5 \mathrm{~Hz}, 2 \mathrm{H}), 1.78-1.64$ $(\mathrm{m}, 2 \mathrm{H})$.

${ }^{13} \mathrm{C}$ NMR (100 MHz, DMSO-d $\left.{ }^{6}\right) \delta 155.49,153.86,141.76,133.73,128.33,128.31,125.75,119.36$, 113.87, 55.14, 38.72, 32.54, 31.69.

IR (neat) v: 1642, 1602, 1564, 1512, 1244, $832 \mathrm{~cm}^{-1}$.

HRMS (ESI) calcd for $\mathrm{C}_{17} \mathrm{H}_{21} \mathrm{~N}_{2} \mathrm{O}_{2}: 285.15975$, found: 285.16036 .

1-(3-phenylpropyl)-3-(4-(trifluoromethyl)phenyl)urea (5g)

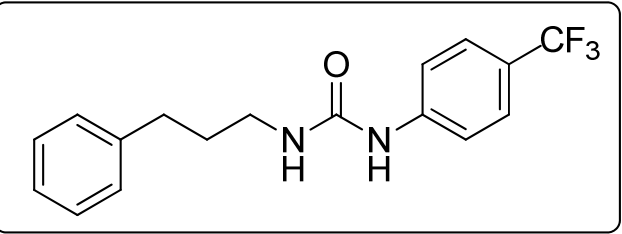

White solid. (69mg, 54\%)

${ }^{1} \mathrm{H}$ NMR $\left(300 \mathrm{MHz}, \mathrm{CDCl}_{3}\right) \delta 8.12(\mathrm{~s}, 1 \mathrm{H}), 7.41-7.29(\mathrm{~m}, 4 \mathrm{H}), 7.26-7.09(\mathrm{~m}, 3 \mathrm{H}), 7.02(\mathrm{~d}, J=6.9$ $\mathrm{Hz}, 2 \mathrm{H}), 6.04(\mathrm{~s}, 1 \mathrm{H}), 3.20-3.13(\mathrm{~m}, 2 \mathrm{H}), 2.52(\mathrm{t}, J=7.6 \mathrm{~Hz}, 2 \mathrm{H}), 1.81-1.63(\mathrm{~m}, 2 \mathrm{H})$.

${ }^{13} \mathrm{C}$ NMR $\left(75 \mathrm{MHz}, \mathrm{CDCl}_{3}\right) \delta 156.47,142.31,141.10,128.60,128.31,126.38$ (q, $\left.J=5.0 \mathrm{~Hz}\right), 124.83$ $(\mathrm{q}, J=32.7 \mathrm{~Hz}), 124.30(\mathrm{q}, J=269.7 \mathrm{~Hz}), 119.04,39.93,33.15,31.63$.

${ }^{19} \mathrm{~F} \mathrm{NMR}\left(376 \mathrm{MHz}, \mathrm{CDCl}_{3}\right) \delta-62.0$.

IR (neat) v: 1657, 1604, 1557, 1328, 1119, 1068, $656 \mathrm{~cm}^{-1}$.

HRMS (ESI) calcd for $\mathrm{C}_{17} \mathrm{H}_{18} \mathrm{~F}_{3} \mathrm{~N}_{2} \mathrm{O}: 323.13657$, found: 323.13569 .

1-(naphthalen-2-yl)-3-(3-phenylpropyl)urea (5h)

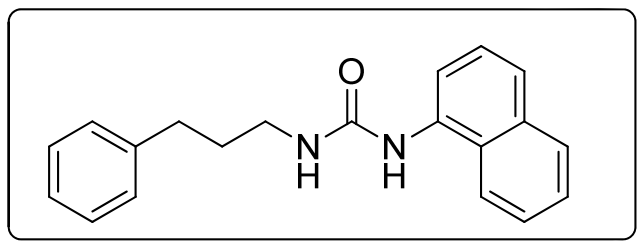

White solid. (95mg, 78\%)

${ }^{1} \mathrm{H}$ NMR (400 MHz, DMSO-d $\left.{ }^{6}\right) \delta 8.52(\mathrm{~s}, 1 \mathrm{H}), 8.11-8.09(\mathrm{~m}, 1 \mathrm{H}), 8.01-7.99(\mathrm{~m}, 1 \mathrm{H}), 7.90-7.88(\mathrm{~m}$, $1 \mathrm{H})$, 7.57-7.49 (m, 3H), 7.43-7.39 (m, 1H), 7.31-7.28 (m, 2H), 7.25-7.23 (m, 2H), 7.21-7.16 (m, 1H), $6.67(\mathrm{t}, J=5.6 \mathrm{~Hz}, 1 \mathrm{H}), 3.19-3.14(\mathrm{~m}, 2 \mathrm{H}), 2.67-2.63(\mathrm{~m}, 2 \mathrm{H}), 1.82-1.75(\mathrm{~m}, 2 \mathrm{H})$.

${ }^{13} \mathrm{C}$ NMR (100 MHz, DMSO-d $\left.{ }^{6}\right) \delta 155.57,141.68,135.20,133.70,128.33,128.30,128.29,125.91$, $125.74,125.70,125.46,125.34,121.91,121.35,116.32,38.76,32.52,31.53$. 
IR (neat) v: 2958, 2083, 1692, 1381, 1300, $1127 \mathrm{~cm}^{-1}$.

HRMS (ESI) calcd for: 305.16484, found: 305.16422.

1-phenethyl-3-(3-phenylpropyl)urea (5i)<smiles>O=C(NCCCc1ccccc1)NCCc1ccccc1</smiles>

White solid. (92mg, 81\%)

${ }^{1} \mathrm{H}$ NMR (400 MHz, $\mathrm{CDCl}_{3}$ ) $\delta$ 7.31-7.14 (m, 10H), 4.43 (s, 2H), 3.40-3.37 (m, 2H), 3.15-3.13 (m, 2H), 2.80-2.76 (m, 2H), 2.64-2.60 (m, 2H), 1.82-1.75 (m, 2H).

${ }^{13} \mathrm{C}$ NMR $\left(100 \mathrm{MHz}, \mathrm{CDCl}_{3}\right) \delta 158.27,141.69,139.31,128.94,128.71,128.56,128.48,126.53$, 126.07, 41.77, 40.23, 36.53, 33.32, 31.92 .

IR (neat) v: 2927, 2859, 1643, 1564, 1496, 1453, $835 \mathrm{~cm}^{-1}$.

HRMS (ESI) calcd for $\mathrm{C}_{18} \mathrm{H}_{23} \mathrm{~N}_{2} \mathrm{O} 283.18049$, found: 283.17970 .

N-phenethylmorpholine-4-carboxamide (5j) ${ }^{8}$

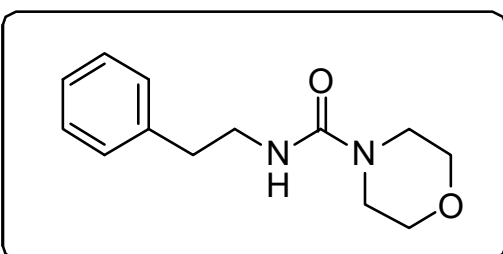

White solid. (75mg, 80\%)

${ }^{1} \mathrm{H}$ NMR (400 MHz, $\left.\mathrm{CDCl}_{3}\right) \delta$ 7.33-7.29 (m, 2H), 7.25-7.19 (m, 3H), $4.46(\mathrm{~s}, 1 \mathrm{H}), 3.66-3.64(\mathrm{~m}, 4 \mathrm{H})$, 3.53-3.48 (m, 2H), 3.29-3.26 (m, 4H), 2.85-2.81 (m, 2H).

${ }^{13} \mathrm{C} \mathrm{NMR}\left(100 \mathrm{MHz}, \mathrm{CDCl}_{3}\right) \delta 157.79,139.37,128.94,128.74,126.58,66.60,44.04,42.11,36.39$.

1-(3-((tert-butyldimethylsilyl)oxy)propyl)-3-(3-phenylpropyl)urea (5k)

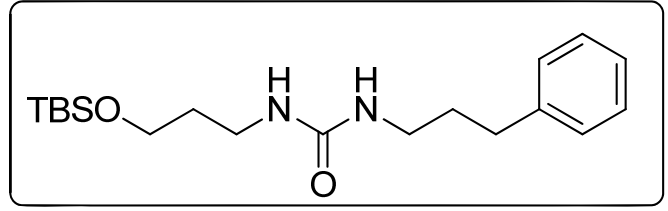

White oil. (103mg, 73\%)

${ }^{1} \mathrm{H}$ NMR (400 MHz, $\left.\mathrm{CDCl}_{3}\right) \delta$ 7.27-7.16 (m, 5H), $4.73(\mathrm{~s}, 1 \mathrm{H}), 4.44(\mathrm{~s}, 1 \mathrm{H}), 3.72-3.69(\mathrm{~m}, 2 \mathrm{H})$, 3.26-3.20 (m, 2H), 2.67-2.63 (m, 2H), 1.84-1.83(m, 2H), 1.70-1.67 (m,4H), $0.90(\mathrm{~s}, 9 \mathrm{H}), 0.06(\mathrm{~s}, 6 \mathrm{H})$.

${ }^{13} \mathrm{C}$ NMR $\left(100 \mathrm{MHz}, \mathrm{CDCl}_{3}\right) \delta 158.54,141.76,128.56,128.50,126.05,61.77,40.33,38.77,34.35$, $32.65,32.05,26.08,18.41,-5.21$.

IR (neat) v: 2928, 2856, 1631, 1575, 1255, 1097, $835 \mathrm{~cm}^{-1}$.

HRMS (ESI) calcd for $\mathrm{C}_{19} \mathrm{H}_{35} \mathrm{~N}_{2} \mathrm{O}_{2} \mathrm{Si}$ : 351.24623, found: 351.24535 . 
$N$-cinnamylmorpholine-4-carboxamide (5l)

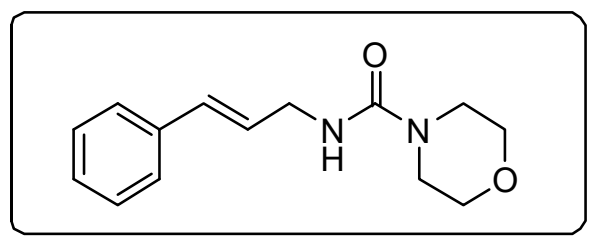

White oil. (51mg, 52\%)

${ }^{1} \mathrm{H}$ NMR $\left(400 \mathrm{MHz}, \mathrm{CDCl}_{3}\right) \delta$ 7.37-7.21 (m, 5H), 6.53-6.49 (m, 1H), 6.27-6.20 (m, 1H), $4.65(\mathrm{~s}, 1 \mathrm{H})$, 4.04-4.01(m, 2H), 3.69-3.67 (m, 4H), 3.37-3.35 (m, 4H).

${ }^{13} \mathrm{C}$ NMR $\left(100 \mathrm{MHz}, \mathrm{CDCl}_{3}\right) \delta 157.53,136.58,131.74,128.54,127.63,126.69,126.31,66.48,43.98$, 43.07.

IR (neat) v: 2921, 2856, 1629, 1534, 1259, 1117, $966 \mathrm{~cm}^{-1}$.

HRMS (ESI) calcd for $\mathrm{C}_{14} \mathrm{H}_{18} \mathrm{~N}_{2} \mathrm{NaO}_{2}: 269.12605$, found: 269.12557 .

1-(4-methoxyphenyl)-3-phenylurea (7a) ${ }^{9}$

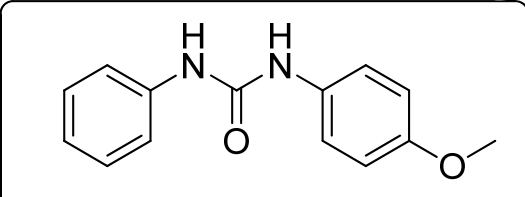

Yellow solid. (88mg, 90\%)

${ }^{1} \mathrm{H}$ NMR (300 MHz, DMSO-d $\left.{ }^{6}\right) \delta 8.56(\mathrm{~s}, 1 \mathrm{H}), 8.45(\mathrm{~s}, 1 \mathrm{H}), 7.44(\mathrm{~d}, J=7.8 \mathrm{~Hz}, 2 \mathrm{H}), 7.35(\mathrm{~d}, J=9.0$ $\mathrm{Hz}, 2 \mathrm{H}), 7.29-7.23$ (m, 2H), 6.97-6.92 (m, 1H), 6.90-6.81 (m, 2H), 3.71 (s, 3H).

${ }^{13} \mathrm{C}$ NMR (75 MHz, DMSO-d $\left.{ }^{6}\right) \delta 154.46,152.70,139.87,132.70,128.70,121.56,120.01,118.06$, $113.97,55.15$.

1-(4-methoxyphenyl)-3-(o-tolyl)urea (7b) ${ }^{10}$

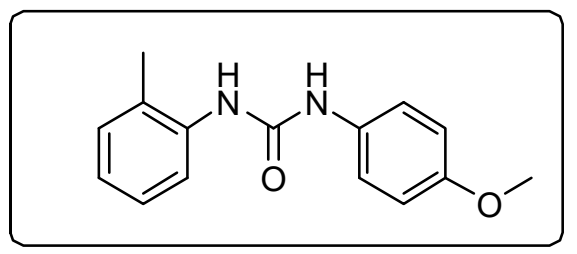

Yellow solid. (93mg, 91\%)

${ }^{1} \mathrm{H}$ NMR (400 MHz, DMSO-d $\left.{ }^{6}\right) \delta 8.82(\mathrm{~s}, 1 \mathrm{H}), 7.84-7.81(\mathrm{~m}, 1 \mathrm{H}), 7.81(\mathrm{~s}, 1 \mathrm{H}), 7.37-7.35(\mathrm{~m}, 2 \mathrm{H})$, 7.17-7.11 (m, 2H), 6.92-6.90 (m, 1H), 6.88-6.86 (m, 2H), 3.71 (s, 3H), 2.23 (s, 3H).

${ }^{13} \mathrm{C}$ NMR (100 MHz, DMSO-d $\left.{ }^{6}\right) \delta 154.32,152.78,137.59,132.91,130.12,127.19,126.11,122.39$, $120.78,119.70,114.01,55.15,17.89$.

1-(4-methoxyphenyl)-3-(m-tolyl)urea (7c) ${ }^{10}$

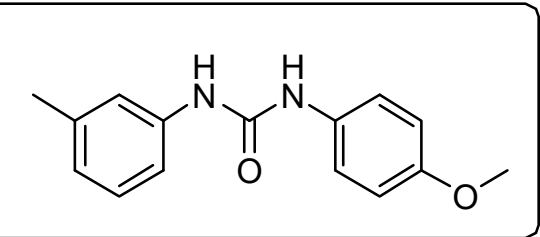


Yellow solid. (84mg, 81\%)

${ }^{1} \mathrm{H}$ NMR (400 MHz, DMSO-d $\left.{ }^{6}\right) \delta 8.48(\mathrm{~s}, 1 \mathrm{H}), 8.43(\mathrm{~s}, 1 \mathrm{H}), 7.35-7.33(\mathrm{~m}, 2 \mathrm{H}), 7.28-7.27(\mathrm{~m}, 1 \mathrm{H})$, $7.21(\mathrm{~d}, J=8.0 \mathrm{~Hz}, 1 \mathrm{H}), 7.15-7.11(\mathrm{~m}, 1 \mathrm{H}), 6.87-6.85(\mathrm{~m}, 2 \mathrm{H}), 6.77(\mathrm{~d}, J=4.0 \mathrm{~Hz}, 1 \mathrm{H}), 3.71(\mathrm{~s}, 3 \mathrm{H})$, $2.27(\mathrm{~s}, 3 \mathrm{H})$.

${ }^{13} \mathrm{C}$ NMR (100 MHz, DMSO-d $\left.{ }^{6}\right) \delta 154.40,152.67,139.79,137.86,132.73,128.56,122.32,119.92$, $118.56,115.23,113.96,55.16,21.23$.

1-(4-methoxyphenyl)-3-(p-tolyl)urea (7d) ${ }^{11}$

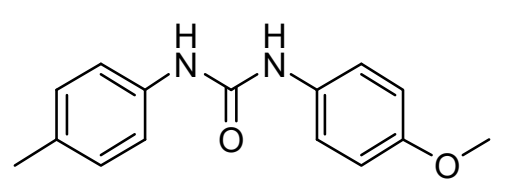

Yellow solid. (95mg, 93\%)

${ }^{1} \mathrm{H}$ NMR (400 MHz, DMSO-d $\left.{ }^{6}\right) \delta 8.44(\mathrm{~s}, 1 \mathrm{H}), 8.40(\mathrm{~s}, 1 \mathrm{H}), 7.35-7.30(\mathrm{~m}, 4 \mathrm{H}), 7.06(\mathrm{~d}, J=8.0 \mathrm{~Hz}$, $2 \mathrm{H}), 6.85(\mathrm{~d}, J=8.0 \mathrm{~Hz}, 2 \mathrm{H}), 3.71(\mathrm{~s}, 3 \mathrm{H}), 2.23(\mathrm{~s}, 3 \mathrm{H})$.

${ }^{13} \mathrm{C}$ NMR (100 MHz, DMSO-d $\left.{ }^{6}\right) \delta 154.37,152.77,137.33,132.83,130.37,129.14,119.92,118.16$, $113.97,55.16,20.35$.

1,3-bis(4-methoxyphenyl)urea (7e) ${ }^{12}$

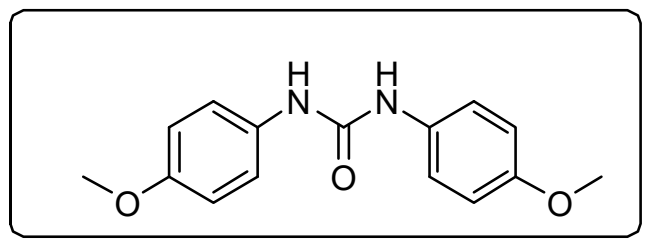

Yellow solid. (96mg, 88\%)

${ }^{1} \mathrm{H}$ NMR (400 MHz, DMSO-d $\left.{ }^{6}\right) \delta 8.35$ (s, 2H), $7.32(\mathrm{~d}, J=8.0 \mathrm{~Hz}, 4 \mathrm{H}), 6.84(\mathrm{~d}, J=8.0 \mathrm{~Hz}, 4 \mathrm{H}), 3.70$ $(\mathrm{s}, 6 \mathrm{H})$.

${ }^{13} \mathrm{C}$ NMR (100 MHz, DMSO-d $\left.{ }^{6}\right) \delta 154.73,153.36,133.34,120.31,114.38,55.59$.

1-(4-acetylphenyl)-3-(4-methoxyphenyl)urea (7f) ${ }^{13}$

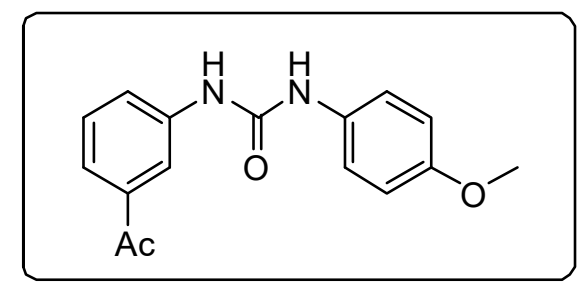

Yellow solid. (89mg, 78\%)

${ }^{1} \mathrm{H}$ NMR (400 MHz, DMSO-d $\left.{ }^{6}\right) \delta 8.81(\mathrm{~s}, 1 \mathrm{H}), 8.51(\mathrm{~s}, 1 \mathrm{H}), 8.06-8.05(\mathrm{~m}, 1 \mathrm{H}), 7.69-7.64(\mathrm{~m}, 1 \mathrm{H})$, $7.56(\mathrm{~d}, J=7.8 \mathrm{~Hz}, 1 \mathrm{H}), 7.44-7.36(\mathrm{~m}, 1 \mathrm{H}), 7.38-7.34(\mathrm{~m}, 2 \mathrm{H}), 6.89-6.84(\mathrm{~m}, 2 \mathrm{H}), 3.72(\mathrm{~s}, 3 \mathrm{H}), 2.56(\mathrm{~s}$, $3 \mathrm{H})$. 
${ }^{13} \mathrm{C}$ NMR (100 MHz, DMSO-d $\left.{ }^{6}\right) \delta 198.19,155.01,153.15,140.77,137.80,132.91,129.54,123.09$, $122.11,120.65,117.66,114.40,55.60,27.19$.

methyl 4-(3-(4-methoxyphenyl)ureido)benzoate (7g)

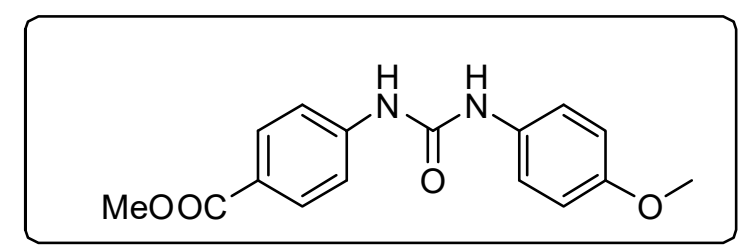

White solid. (106mg, 88\%)

${ }^{1} \mathrm{H}$ NMR (400 MHz, DMSO-d $\left.{ }^{6}\right) \delta 9.01(\mathrm{~s}, 1 \mathrm{H}), 8.60(\mathrm{~s}, 1 \mathrm{H}), 7.87(\mathrm{~d}, J=8.6 \mathrm{~Hz}, 2 \mathrm{H}), 7.57$ (d, $J=8.6$ $\mathrm{Hz}, 2 \mathrm{H}), 7.36(\mathrm{~d}, J=8.8 \mathrm{~Hz}, 2 \mathrm{H}), 6.88(\mathrm{~d}, J=8.8 \mathrm{~Hz}, 2 \mathrm{H}), 3.81(\mathrm{~s}, 3 \mathrm{H}), 3.72(\mathrm{~s}, 3 \mathrm{H})$.

${ }^{13} \mathrm{C}$ NMR (100 MHz, DMSO-d $\left.{ }^{6}\right) \delta 165.92,154.72,152.30,144.59,132.24,130.38,122.17,120.26$, $117.15,114.01,55.17,51.75$.

IR (neat) v: 1714, 1651, 1600, 1563, 1512, 1284, $832 \mathrm{~cm}^{-1}$.

HRMS (ESI) calcd for $\mathrm{C}_{16} \mathrm{H}_{17} \mathrm{~N}_{2} \mathrm{O}_{4}: 301.11828$, found: 301.11840 .

1-(4-methoxyphenyl)-3-(4-(trifluoromethyl)phenyl)urea (7h) ${ }^{14}$

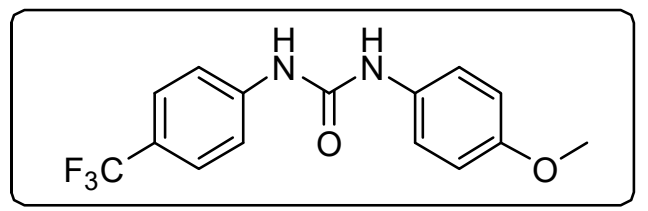

White solid. (120mg, 97\%)

${ }^{1} \mathrm{H}$ NMR (400 MHz, DMSO-d $\left.{ }^{6}\right) \delta 9.01(\mathrm{~s}, 1 \mathrm{H}), 8.60(\mathrm{~s}, 1 \mathrm{H}), 7.66-7.60(\mathrm{~m}, 4 \mathrm{H}), 7.36(\mathrm{~d}, J=8.0 \mathrm{~Hz}$, $2 \mathrm{H}), 6.88(\mathrm{~d}, J=8.0 \mathrm{~Hz}, 2 \mathrm{H}), 3.72(\mathrm{~s}, 3 \mathrm{H})$.

${ }^{13} \mathrm{C}$ NMR (100 MHz, DMSO- d $\left.{ }^{6}\right) \delta 154.72,152.43,143.62,132.25,126.05$ (q, $\left.J=4.0 \mathrm{~Hz}\right), 124.59$ (q, $J=270.0 \mathrm{~Hz}), 120.31,118.14,117.69,114.01,55.18$.

${ }^{19} \mathrm{~F}$ NMR (376 MHz, DMSO- $\mathrm{d}^{6}$ ) $\delta-60.1$.

1-(4-fluorophenyl)-3-(4-methoxyphenyl)urea (7i) ${ }^{15}$

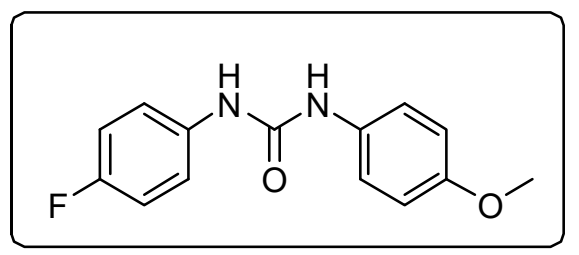

White solid. (97mg, 93\%)

${ }^{1} \mathrm{H}$ NMR (400 MHz, DMSO-d $\left.{ }^{6}\right) \delta 8.59$ (s, 1H), 8.44 (s, 1H), 7.46-7.42 (m, 2H), 7.35-7.33 (m, 2H), 7.12-7.08 (m, 2H), 6.7-6.85 (m, 2H), $3.71(\mathrm{~s}, 3 \mathrm{H})$.

${ }^{13} \mathrm{C}$ NMR(100 MHz, DMSO-d $\left.{ }^{6}\right) \delta 157.19(\mathrm{q}, J=236.2 \mathrm{~Hz}), 154.46,152.81,136.22,132.66,120.07$, 119.79 (d, $J=7.4 \mathrm{~Hz}), 115.21$, (d, $J=21.2 \mathrm{~Hz}), 113.96,55.16$.

${ }^{19}$ F NMR (376 MHz, DMSO- $d^{6}$ ) $\delta$-121.9. 
1-(4-bromophenyl)-3-(4-methoxyphenyl)urea (7j) ${ }^{16}$

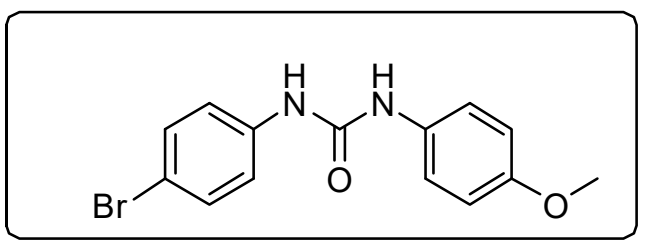

Yellow solid. (89mg, 69\%)

${ }^{1} \mathrm{H}$ NMR (400 MHz, DMSO-d $\left.{ }^{6}\right) \delta 8.72(\mathrm{~s}, 1 \mathrm{H}), 8.50$ (s, 1H), $7.42(\mathrm{~s}, 4 \mathrm{H}), 7.34(\mathrm{~d}, J=8.0 \mathrm{~Hz}, 2 \mathrm{H}), 6.86$ $(\mathrm{d}, J=8.0 \mathrm{~Hz}, 2 \mathrm{H}), 3.71(\mathrm{~s}, 3 \mathrm{H})$.

${ }^{13} \mathrm{C}$ NMR (100 MHz, DMSO-d $\left.{ }^{6}\right) \delta 154.56,152.56,139.34,132.47,131.45,120.15,119.98,113.98$, 112.91, 55.16.

1-(3-chlorophenyl)-3-(4-methoxyphenyl)urea (7k) ${ }^{10}$<smiles>COc1ccc(NC(=O)Nc2cccc(Cl)c2)cc1</smiles>

White solid. (97mg, 87\%)

${ }^{1} \mathrm{H}$ NMR (400 MHz, DMSO-d $\left.{ }^{6}\right) \delta 8.79(\mathrm{~s}, 1 \mathrm{H}), 8.54(\mathrm{~s}, 1 \mathrm{H}), 7.70-7.69(\mathrm{~m}, 1 \mathrm{H}), 7.36-7.33$ (m, 2H), 7.30-7.23 (m, 2H), 7.00-6.98 (m,1H), 6.88-6.86 (m, 2H), $3.72(\mathrm{~s}, 3 \mathrm{H})$.

${ }^{13} \mathrm{C}$ NMR (100 MHz, DMSO-d $\left.{ }^{6}\right) \delta: 154.46,152.54,141.48,133.15,132.36,130.35,121.19,120.24$, 117.40, 116.49, 113.99, 55.17.

1-mesityl-3-(4-methoxyphenyl)urea (7l) ${ }^{17}$

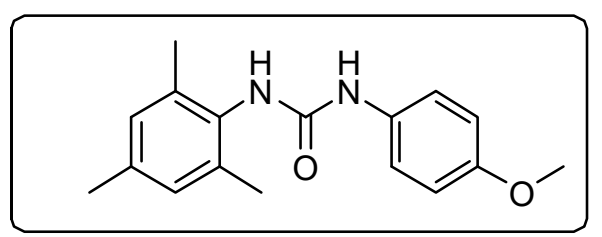

White solid. (93mg, 82\%)

${ }^{1} \mathrm{H}$ NMR (400 MHz, DMSO-d $\left.{ }^{6}\right) \delta 8.45$ (s, 1H), 7.50 (s, 1H), 7.34-7.32 (m, 2H), 6.87 (s, 2H), 6.85-6.82 (m, 2H), 3.70 (s, 3H), 2.22 (s, 3H), 2.15 (s, 6H).

${ }^{13} \mathrm{C}$ NMR (100 MHz, DMSO-d $\left.{ }^{6}\right) \delta 154.07,153.53,135.31,134.75,133.48,132.86,128.29,119.60$, 113.89, 55.1, 20.50, 18.22.

1-(4-methoxyphenyl)-3-(naphthalen-1-yl)urea (7m) ${ }^{18}$<smiles>COc1ccc(NC(=O)Nc2cccc3ccccc23)cc1</smiles> 
White solid. (96mg, 82\%)

${ }^{1} \mathrm{H}$ NMR (400 MHz, DMSO-d $\left.\mathrm{d}^{6}\right) \delta 8.87(\mathrm{~s}, 1 \mathrm{H}), 8.68(\mathrm{~s}, 1 \mathrm{H}), 8.12(\mathrm{~d}, J=7.8 \mathrm{~Hz}, 1 \mathrm{H}), 8.02(\mathrm{~d}, J=7.8 \mathrm{~Hz}$, 1H), 7.94-7.91 (m, 1H), 7.63-7.52 (m, 3H), 7.48-7.44 (m, 1H), 7.43-7.39 (m, 2H), $6.90(\mathrm{~d}, J=9.0 \mathrm{~Hz}$, 2H), 3.73 (s, 3H).

${ }^{13} \mathrm{C}$ NMR (100 MHz, DMSO-d $\left.{ }^{6}\right) \delta 154.46,153.06,134.50,133.71,132.80,128.41,125.88,125.85$, $125.80,125.63,122.66,121.28,119.86,117.09,114.06,55.18$.

phenyl-3-(4-(trifluoromethyl)phenyl)urea (7n) ${ }^{19}$

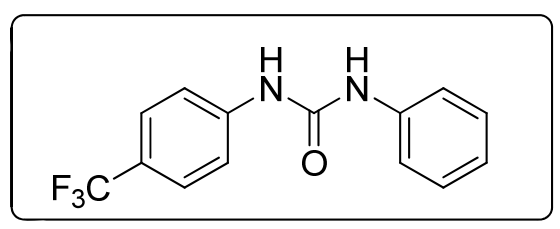

White solid. (99mg, 88\%)

${ }^{1} \mathrm{H}$ NMR (400 MHz, DMSO-d $\left.{ }^{6}\right) \delta 9.08(\mathrm{~s}, 1 \mathrm{H}), 8.78(\mathrm{~s}, 1 \mathrm{H}), 7.67-7.61(\mathrm{~m}, 4 \mathrm{H}), 7.49-7.42(\mathrm{~m}, 2 \mathrm{H})$, 7.31-7.27 (m, 2H), 7.01-6.97 (m, 1H).

${ }^{13} \mathrm{C}$ NMR (100 MHz, DMSO-d ${ }^{6}$ ) $\delta 152.26,143.47,139.28,128.81,126.06$ (q, $\left.J=3.8 \mathrm{~Hz}\right), 124.55$ (q, $J$ $=269.4 \mathrm{~Hz}), 122.20,121.71(\mathrm{q}, J=31.7 \mathrm{~Hz}), 118.43,117.80$.

${ }^{19} \mathrm{~F}$ NMR (376 MHz, DMSO- d $\left.{ }^{6}\right) \delta-60.1$.

1-mesityl-3-phenylurea (7o) ${ }^{18}$

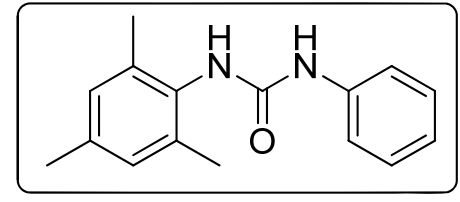

White solid. (46mg, 45\%)

${ }^{1} \mathrm{H}$ NMR (400 MHz, DMSO-d $\left.{ }^{6}\right) 8.66(\mathrm{~s}, 1 \mathrm{H}), 7.60$ (s, 1H), 7.44 (d, J= $\left.7.7 \mathrm{~Hz}, 2 \mathrm{H}\right), 7.26-7.22$ (m, 2H), 6.93-6.90 (m, 1H), $6.88(\mathrm{~s}, 2 \mathrm{H}), 2.23(\mathrm{~s}, 3 \mathrm{H}), 2.16(\mathrm{~s}, 6 \mathrm{H})$.

${ }^{13} \mathrm{C}$ NMR $\left(100 \mathrm{MHz}, \mathrm{CDCl}_{3}\right) \delta 153.70,140.78,135.71,135.28,133.08,129.09,128.72,121.69$, $118.25,20.91,18.60$.

1-butyl-3-phenylurea (7p) ${ }^{20}$

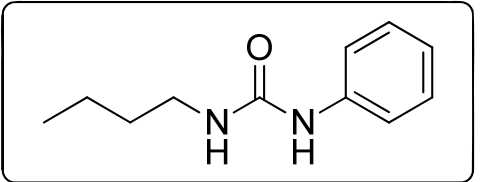

White solid. (66mg, 85\%)

${ }^{1} \mathrm{H}$ NMR $\left(400 \mathrm{MHz}, \mathrm{CDCl}_{3}\right) \quad \delta 8.35(\mathrm{~s}, 1 \mathrm{H}), 7.38-7.35(\mathrm{~m}, 2 \mathrm{H}), 7.23-7.16(\mathrm{~m}, 2 \mathrm{H}), 6.89-6.85(\mathrm{~m}, 1 \mathrm{H})$, $6.08(\mathrm{~s}, 1 \mathrm{H}), 3.09-3.04(\mathrm{~m}, 2 \mathrm{H}), 1.45-1.36(\mathrm{~m}, 2 \mathrm{H}), 1.35-1.27(\mathrm{~m}, 2 \mathrm{H}), 0.89(\mathrm{t}, J=7.2 \mathrm{~Hz}, 3 \mathrm{H})$.

${ }^{13} \mathrm{C} \mathrm{NMR}\left(100 \mathrm{MHz}, \mathrm{CDCl}_{3}\right) \delta 155.16,140.58,128.58,120.83,171.50,38.65,31.87,19.51,13.68$. 
$N$-phenylpiperidine-1-carboxamide (7q) ${ }^{21}$

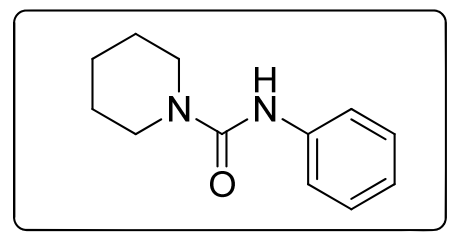

White solid. (82mg, $>98 \%)$

${ }^{1} \mathrm{H}$ NMR (400 MHz, $\left.\mathrm{CDCl}_{3}\right) \delta 8.40(\mathrm{~s}, 1 \mathrm{H}), 7.44(\mathrm{~d}, J=7.8 \mathrm{~Hz}, 2 \mathrm{H}), 7.21(\mathrm{t}, J=7.8 \mathrm{~Hz}, 2 \mathrm{H}), 6.95-6.88$ $(\mathrm{m}, 1 \mathrm{H}), 3.44-3.38(\mathrm{~m}, 4 \mathrm{H}), 1.61-1.52(\mathrm{~m}, 2 \mathrm{H}), 1.51-1.46(\mathrm{~m}, 4 \mathrm{H})$.

${ }^{13} \mathrm{C} \mathrm{NMR}\left(100 \mathrm{MHz}, \mathrm{CDCl}_{3}\right) \delta 154.87,140.77,128.19,121.44,119.54,44.66,25.51,24.12$.

1-methyl-1,3-diphenylurea $(\mathbf{7 r})^{22}$

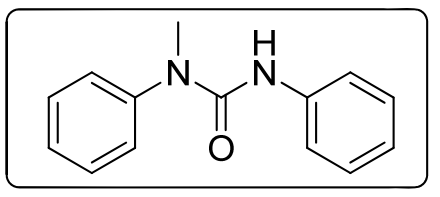

White solid. (84mg, 92\%)

${ }^{1} \mathrm{H}$ NMR (400 MHz, $\left.\mathrm{CDCl}_{3}\right) \delta 8.12(\mathrm{~s}, 1 \mathrm{H}), 7.44-7.37$ (m, 4H), 7.34-7.29 (m, 2H), 7.28-7.18 (m, 3H), 6.96-6.92 (m, 1H), $3.27(\mathrm{~s}, 3 \mathrm{H})$.

${ }^{13} \mathrm{C}$ NMR $\left(100 \mathrm{MHz}, \mathrm{CDCl}_{3}\right) \delta 154.69,144.08,140.05,129.20,128.23,126.18,125.70,121.98$, $119.85,37.52$. 


\section{References:}

(1) Viana, G. M.; Aguiar, L. C. d. S.; Ferrao, J. d. A.; Simas, A. B. C.; Vasconcelos, M. G. Tetrahedron Lett. 2013, 54, 936.

(2) Wu, C.; Cheng, H.; Liu, R.; Wang, Q.; Hao, Y.; Yu, Y.; Zhao, F. Green Chem. 2010, 12, 1811.

(3) Borgna, P.; Vicarini, L.; Calderara, G. Farmaco, Ed. Sci. 1977, 32, 813.

(4) Gann, A. W.; Amoroso, J. W.; Einck, V. J.; Rice, W. P.; Chambers, J. J.; Schnarr, N. A., A Photoinduced, Benzyne Click Reaction. Org. Lett. 2014, 16, 2003-2005.

(5) Kwok, S. W.; Fotsing, J. R.; Fraser, R. J.; Rodionov, V. O.; Fokin, V. V. Org. Lett. 2010, 12, 4217.

(6) Orito, K.; Miyazawa, M.; Nakamura, T.; Horibata, A.; Ushito, H.; Nagasaki, H.; Yuguchi, M.; Yamashita, S.; Yamazaki, T.; Tokuda, M. J. Org. Chem. 2006, 71, 5951.

(7) Skinner, C. G.; Shive, W. J. Org. Chem. 1960, 25, 2046.

(8) Dube, P.; Nathel, N. F. F.; Vetelino, M.; Couturier, M.; Aboussafy, C. L.; Pichette, S.; Jorgensen, M.

L.; Hardink, M. Org. Lett. 2009, 11, 5622.

(9) Kotecki, B. J.; Fernando, D. P.; Haight, A. R.; Lukin, K. A. Org. Lett. 2009, 11, 947.

(10) McKay, A. F.; Hatton, W. G. Can. J. Chem. 1952, 30, 225.

(11) Naegeli, C.; Tyabji, A.; Conrad, L. Helv. Chim. Acta 1938, 21, 1127.

(12) Wei, Y.; Ding, H.; Lin, S.; Liang, F. Org. Lett. 2011, 13, 1674.

(13) Dominguez, J. N.; Leon, C.; Rodrigues, J.; Gamboa de Dominguez, N.; Gut, J.; Rosenthal, P. J. J. Med. Chem. 2005, 48, 3654.

(14) Takano, T.; Uehori, Y.; Hayasaka, H.; Satake, H. JP08002111A, 1996.

(15) Gavade, S. N.; Balaskar, R. S.; Mane, M. S.; Pabrekar, P. N.; Shingare, M. S.; Mane, D. V. Chin.Chem. Lett. 2011, 22, 292.

(16) Picard, J. P.; McKay, A. F. Can. J. Chem. 1953, 31, 896.

(17) Lebel, H.; Leogane, O. Org. Lett. 2006, 8, 5717.

(18) Sammour, A.; Fahmy, A. F. M.; Hosni, G. Egypt. J. Chem. 1973, 16, 481.

(19) Moore, S. J.; Haynes, C. J. E.; Gonzalez, J.; Sutton, J. L.; Brooks, S. J.; Light, M. E.; Herniman, J.; Langley, G. J.; Soto-Cerrato, V.; Perez-Tomas, R.; Marques, I.; Costa, P. J.; Felix, V.; Gale, P. A. Chem. Sci. 2013, 4, 103.

(20) Kotecki, B. J.; Fernando, D. P.; Haight, A. R.; Lukin, K. A. Org Lett 2009, 11, 947.

(21) Wei, Y.; Liu, J.; Lin, S.; Ding, H.; Liang, F.; Zhao, B. Org. Lett. 2010, 12, 4220.

(22) Zhang, L.; Darko, A. K.; Johns, J. I.; McElwee-White, L. Eur. J. Org. Chem. 2011, 31 , 6261. 
7. ${ }^{1} \mathrm{H}$ and ${ }^{13} \mathrm{C}$ NMR spectra
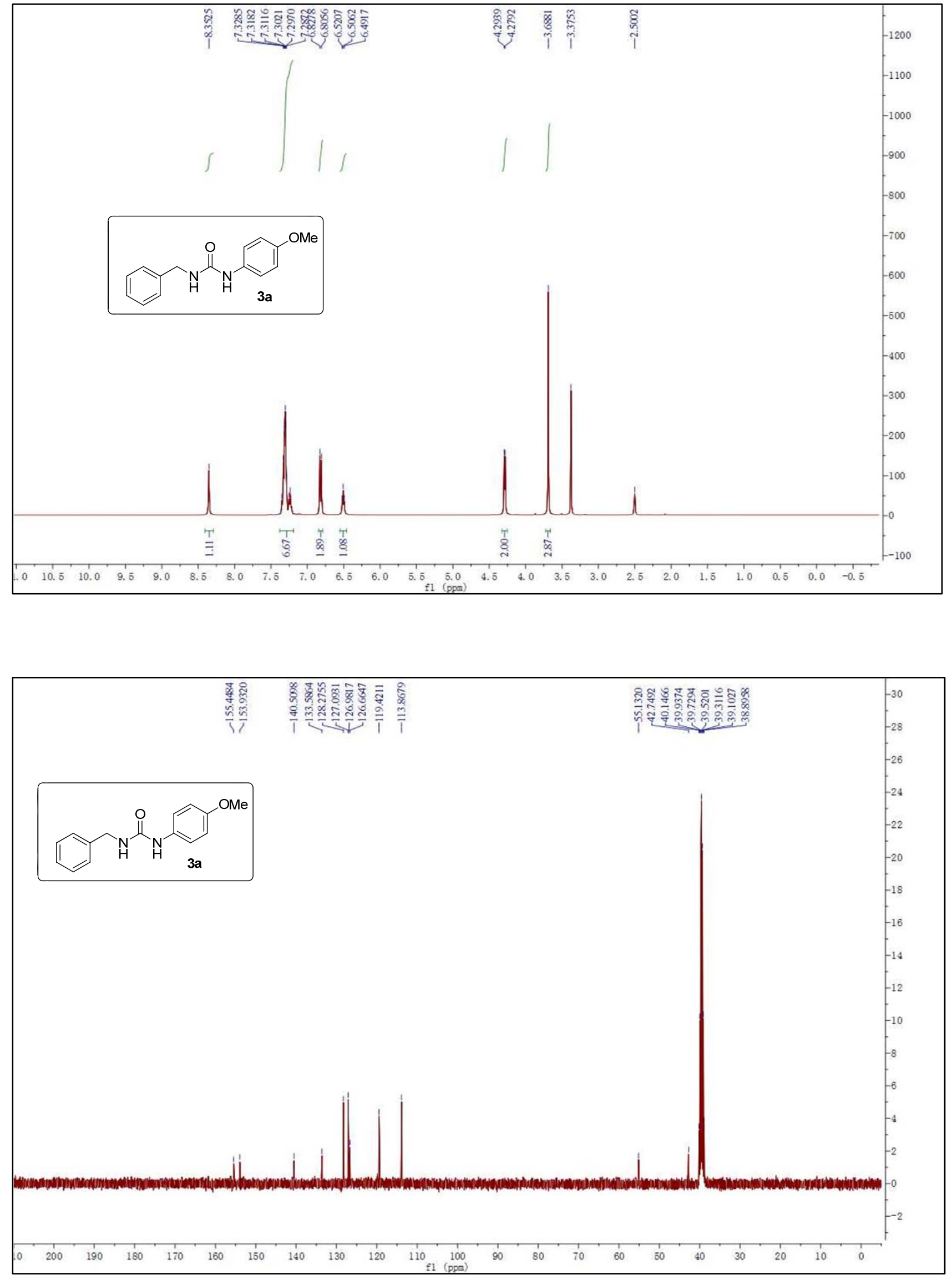

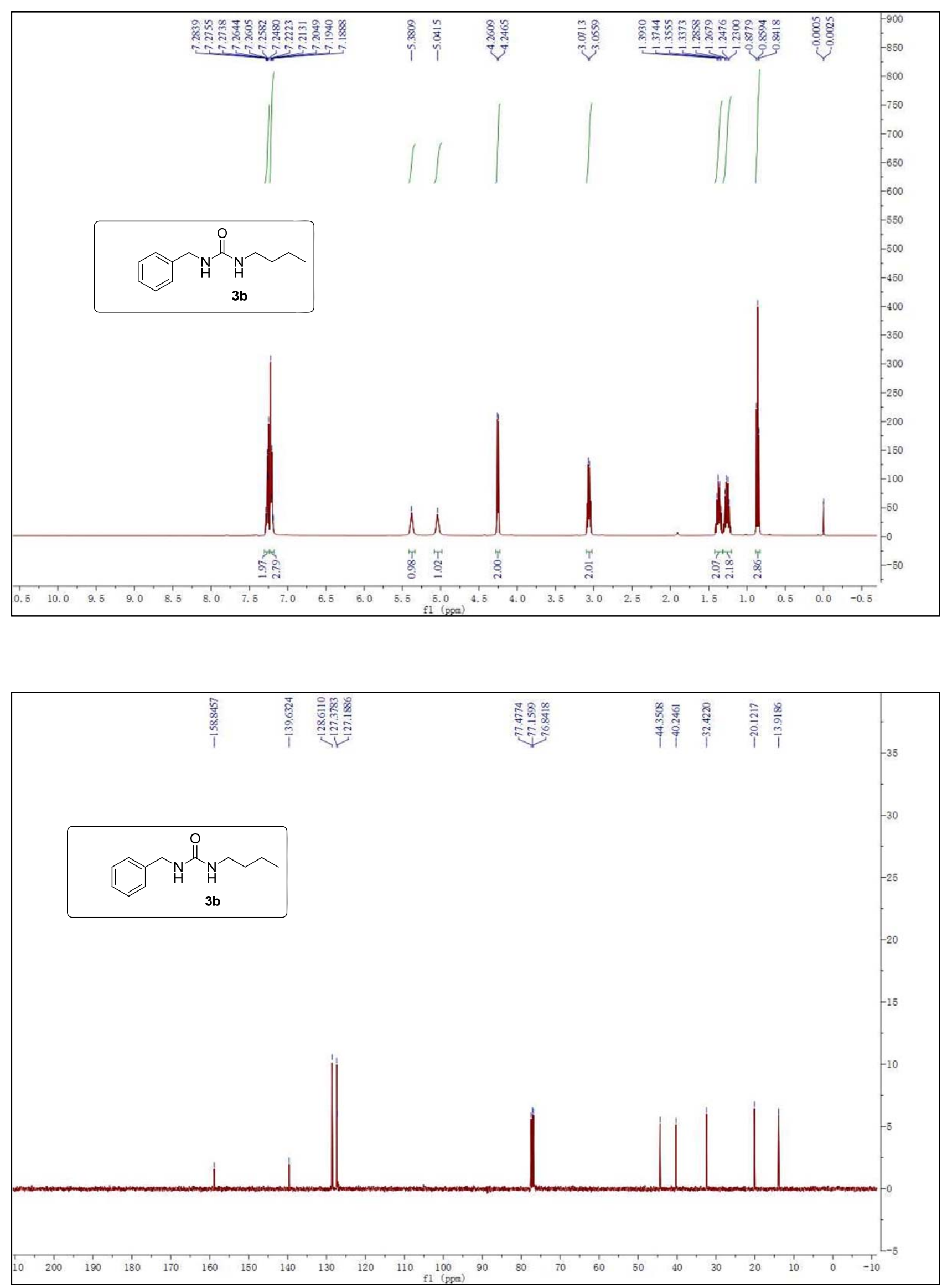

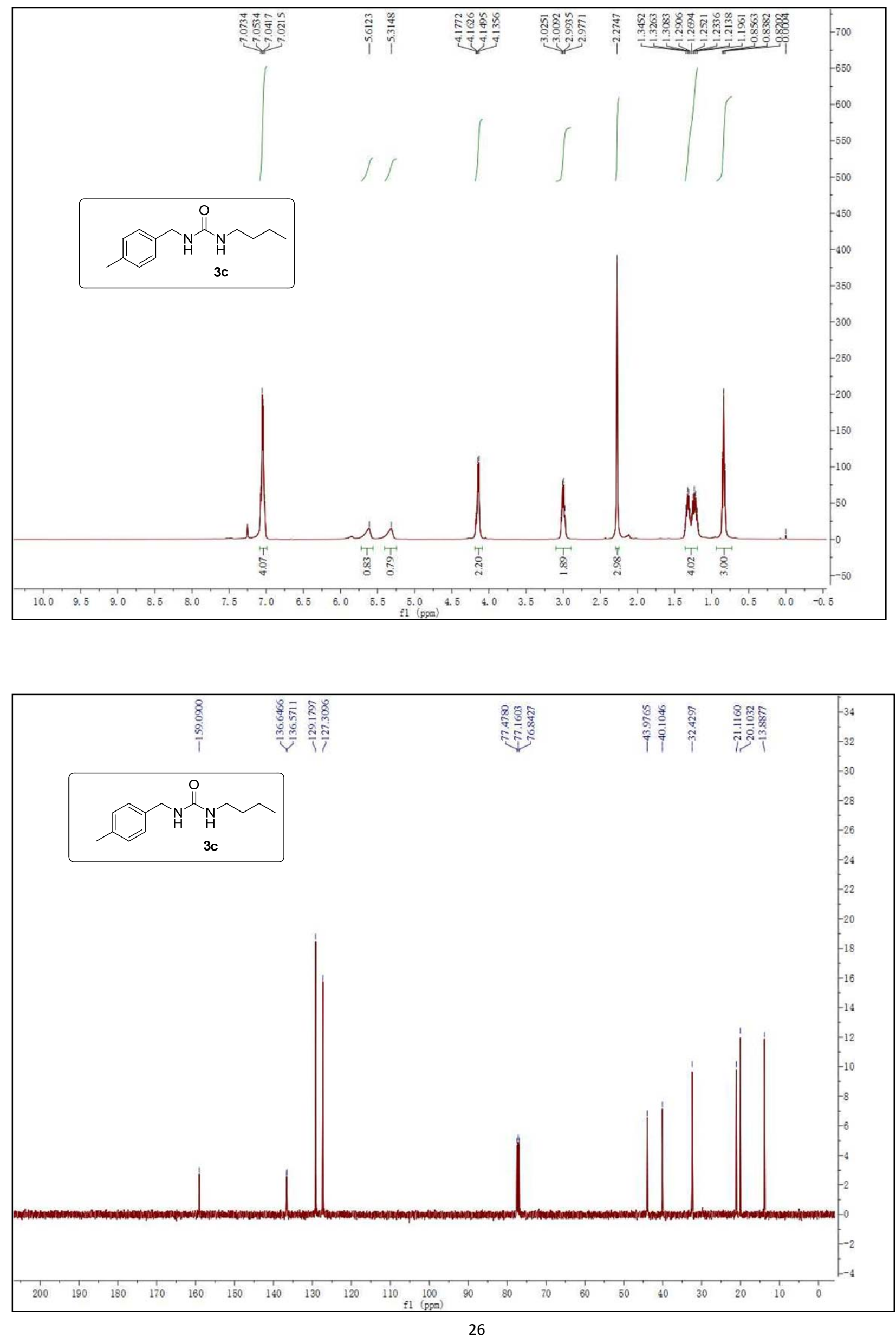

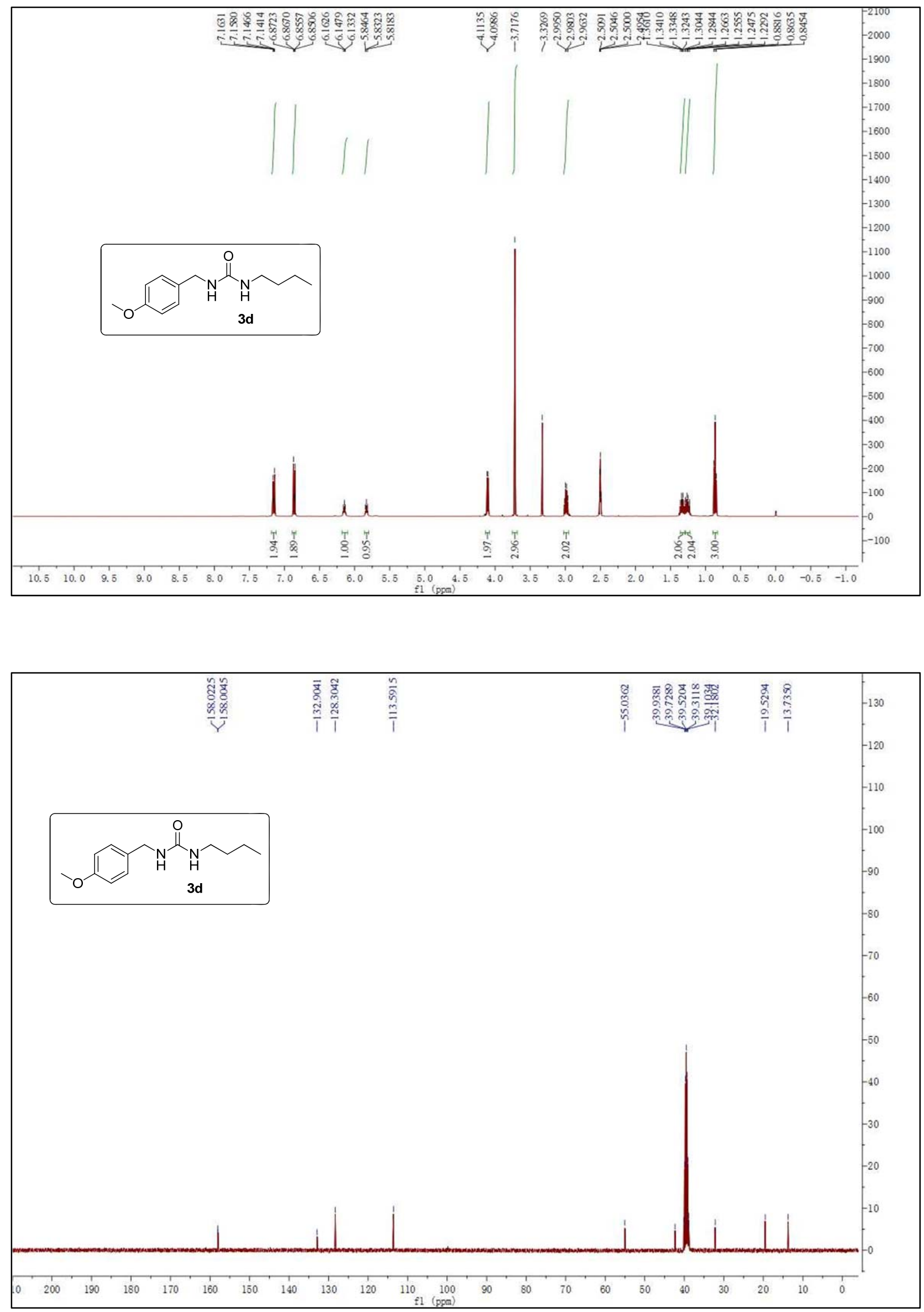

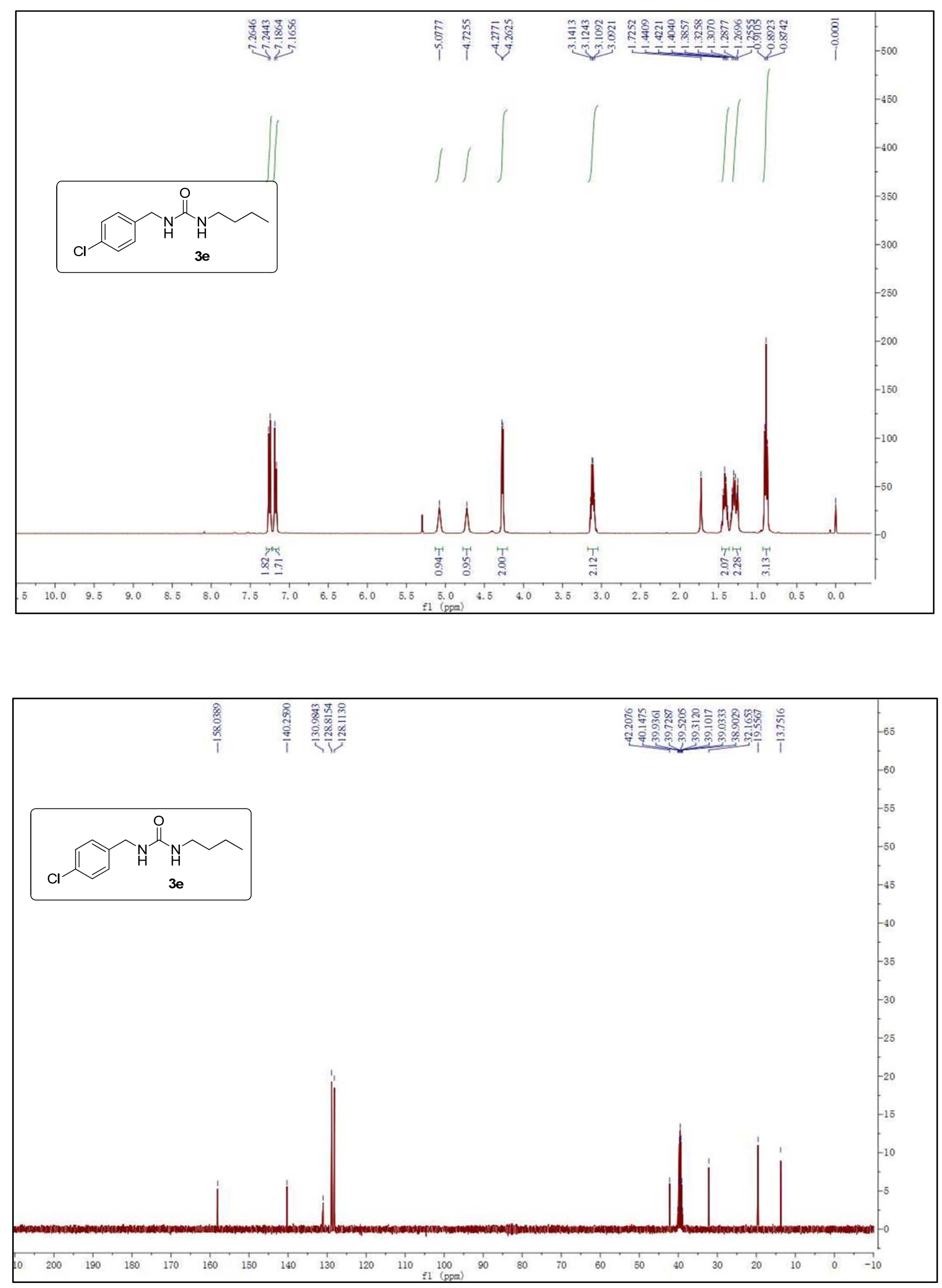

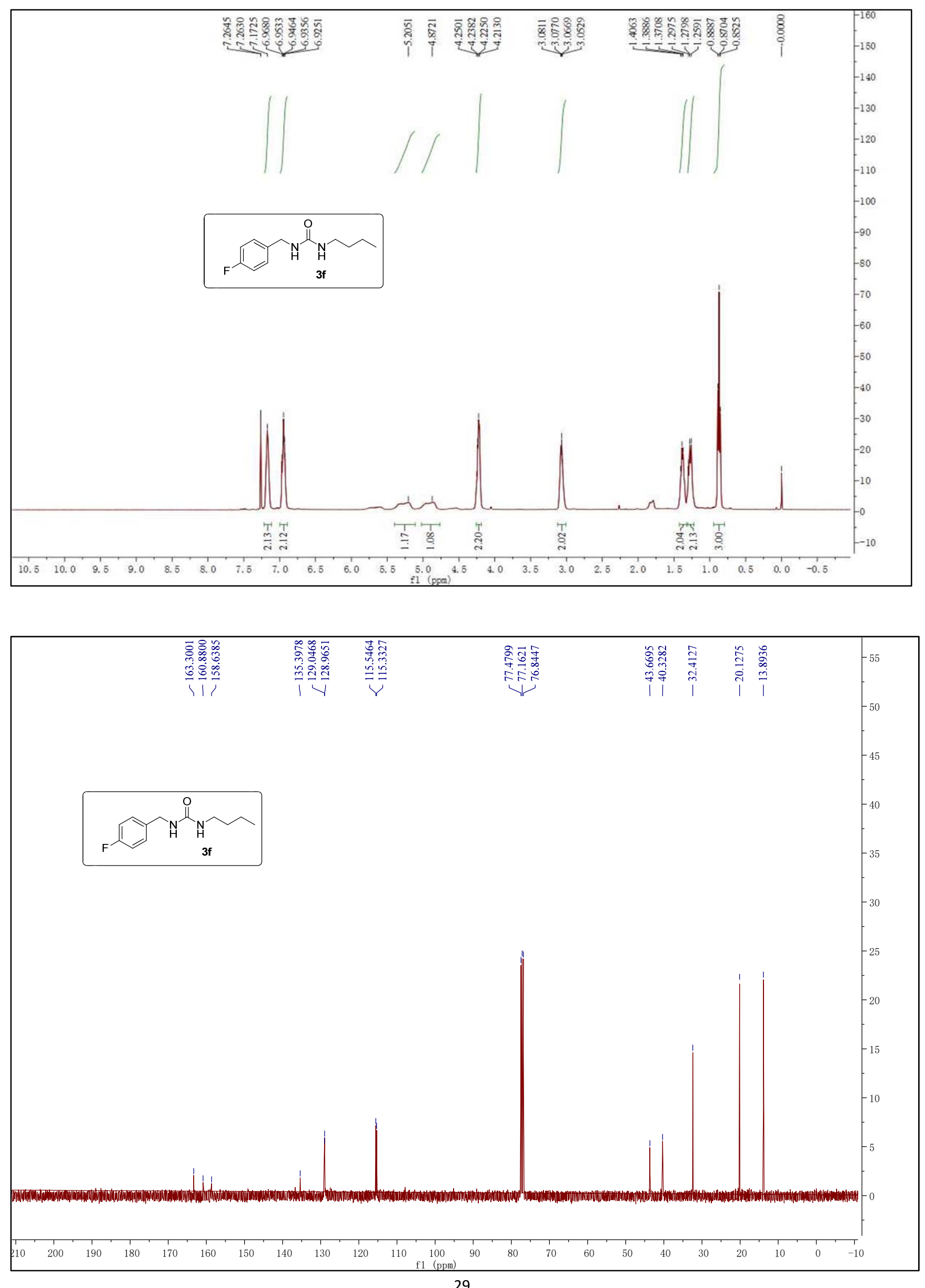

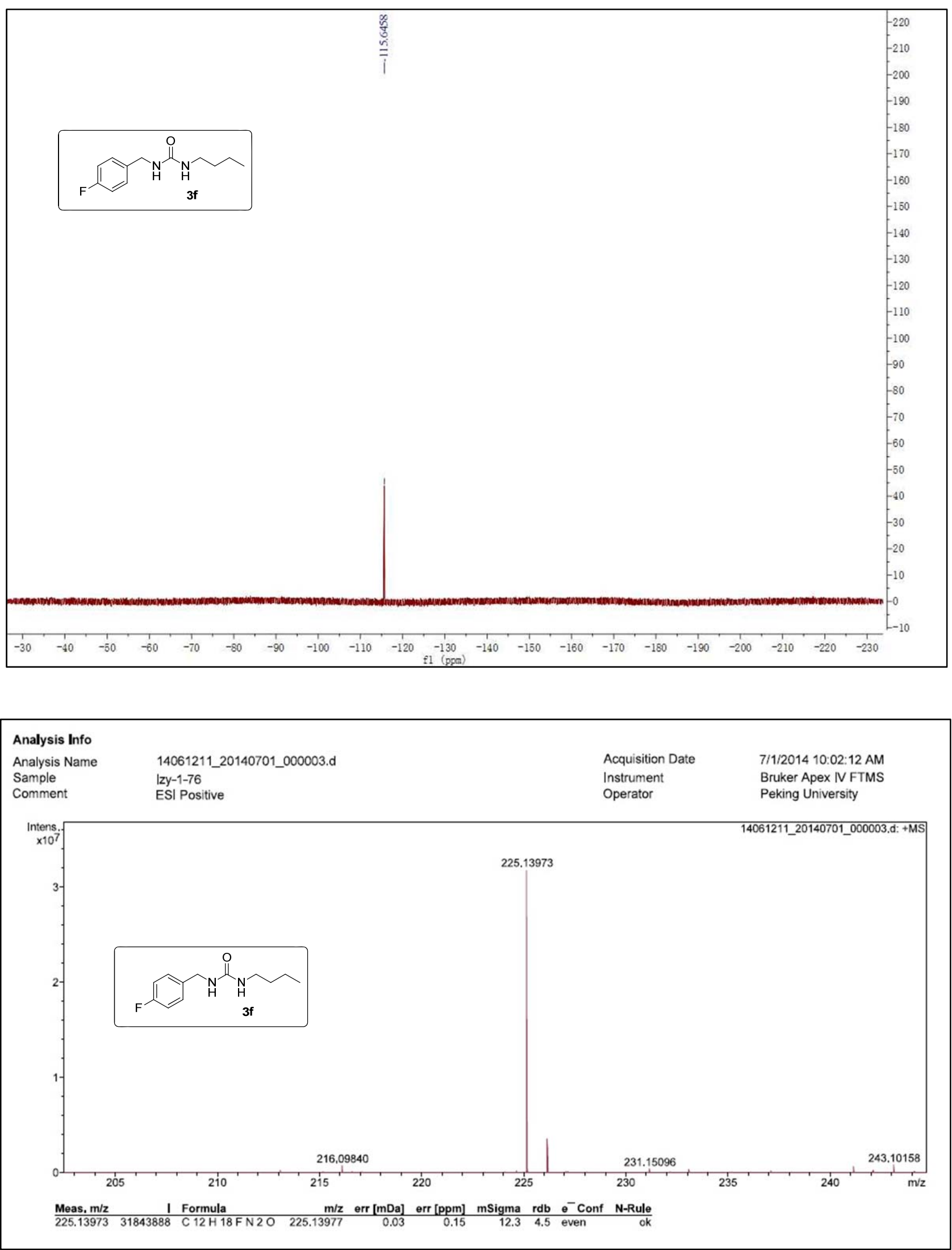

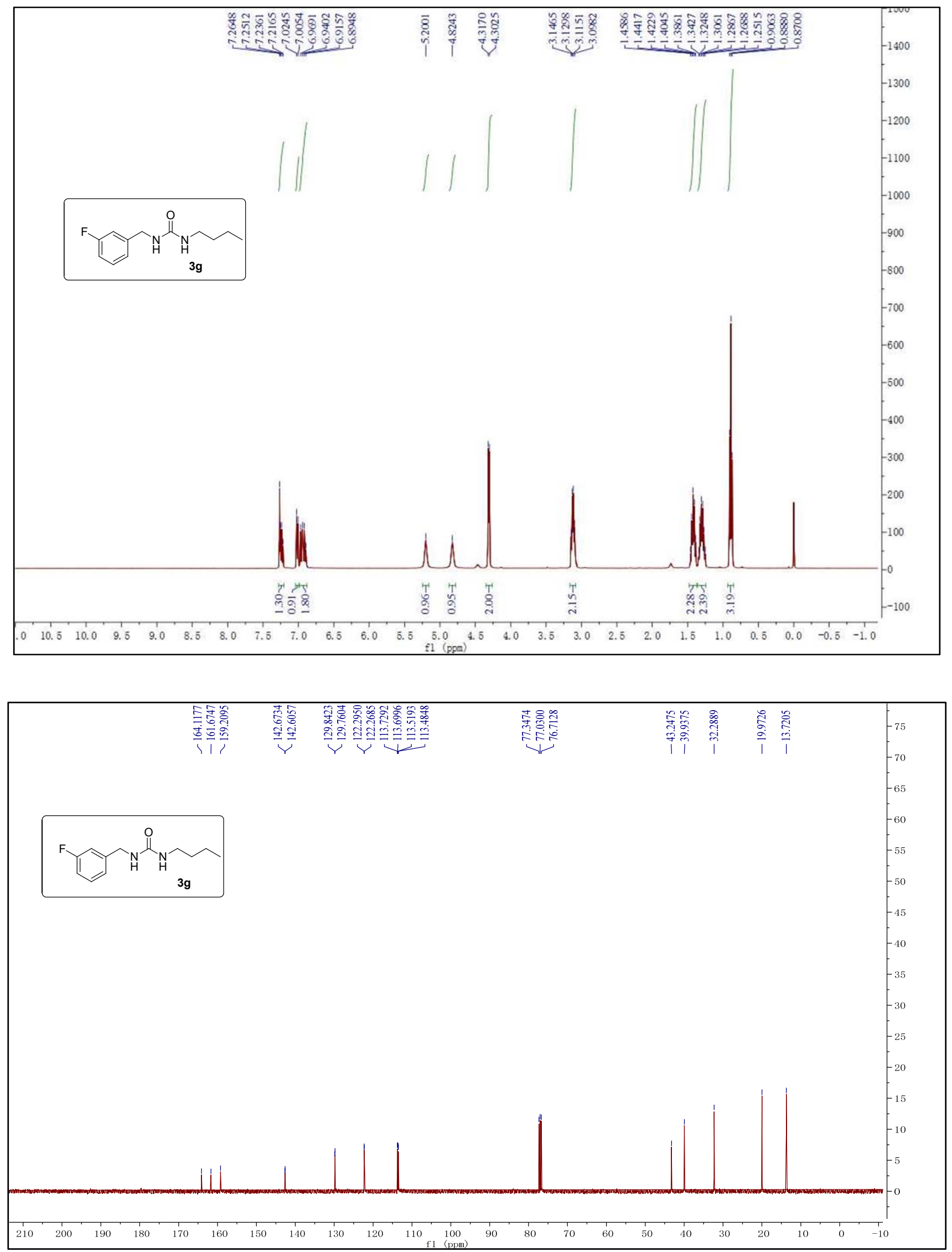

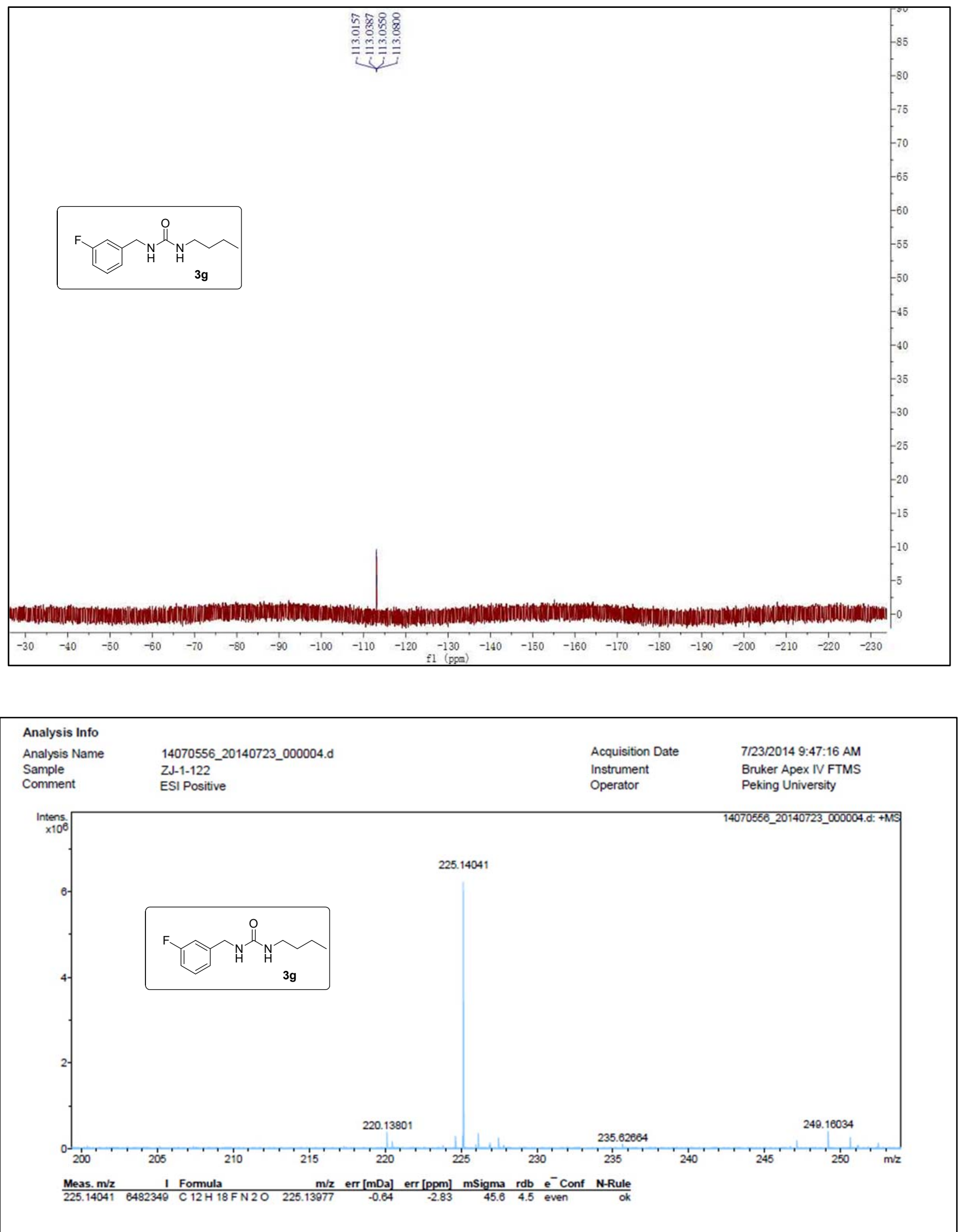

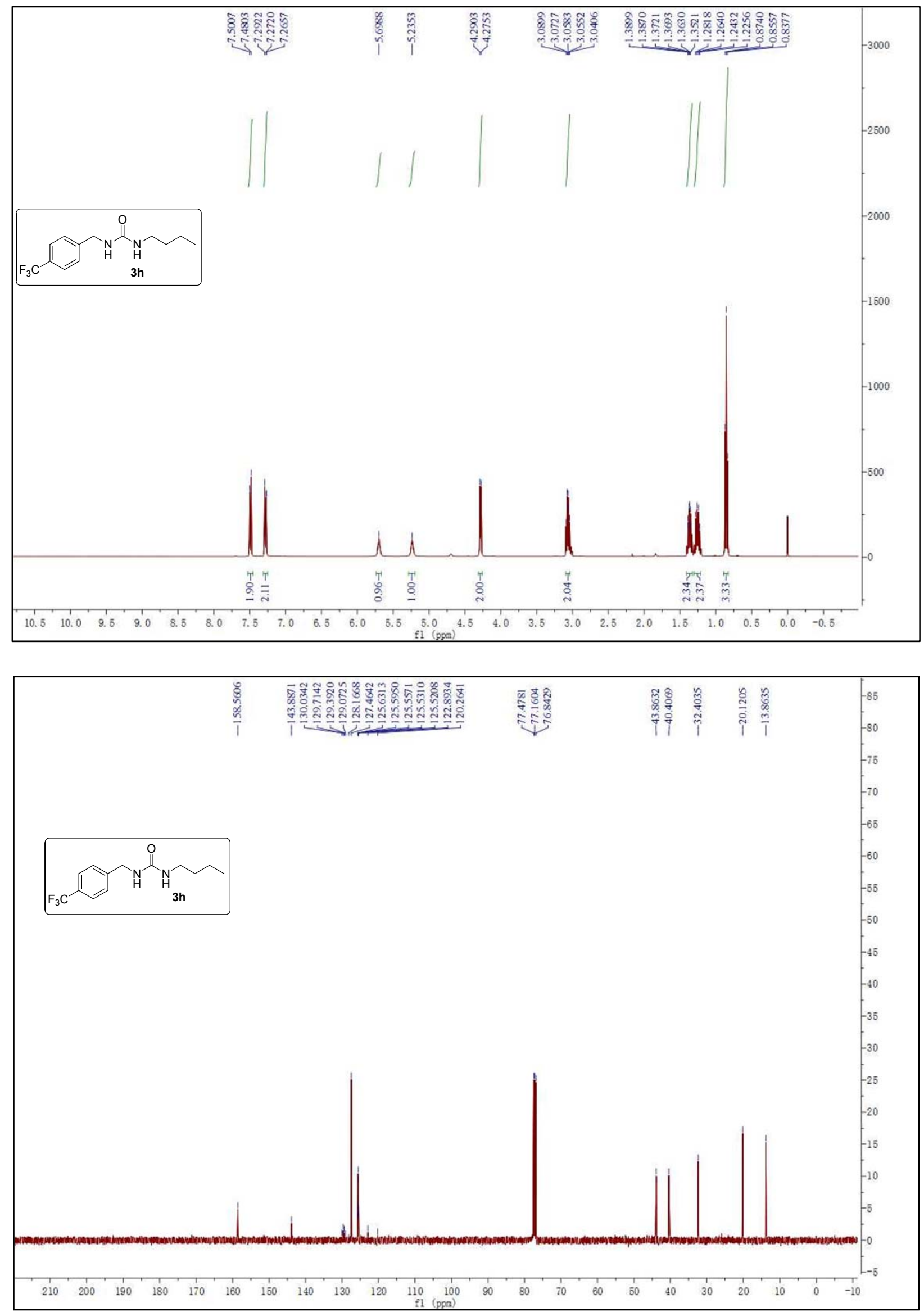


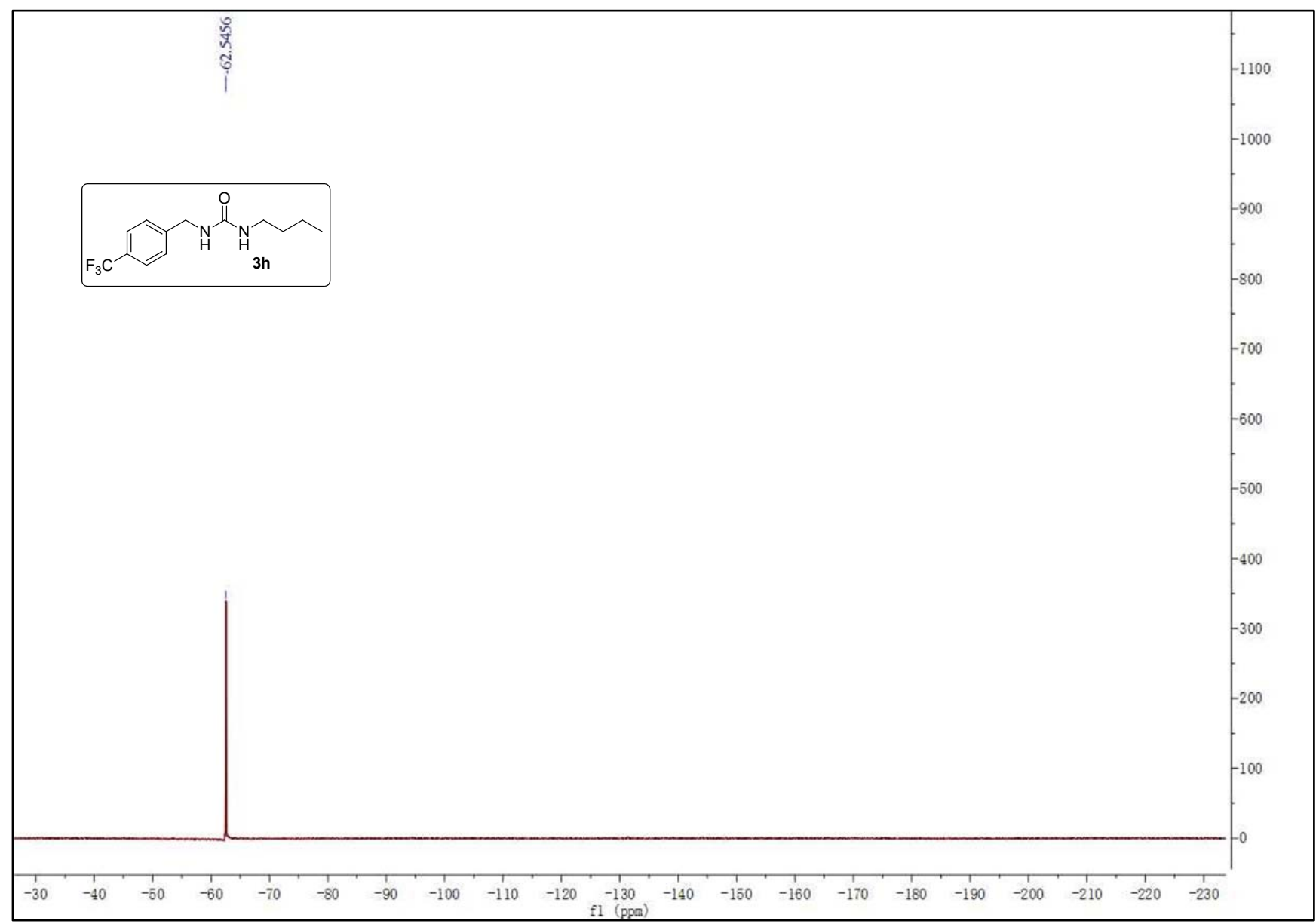

\begin{tabular}{llll}
\hline Analysis Info & & \\
Analysis Name & 14061211 20140701_000005.d & Acquisition Date & $7 / 1 / 2014$ 10:07:33 AM \\
Sample & $\mid z y-1-75$ & Instrument & Bruker Apex IV FTMS \\
Comment & ESI Positive & Operator & Peking University
\end{tabular}

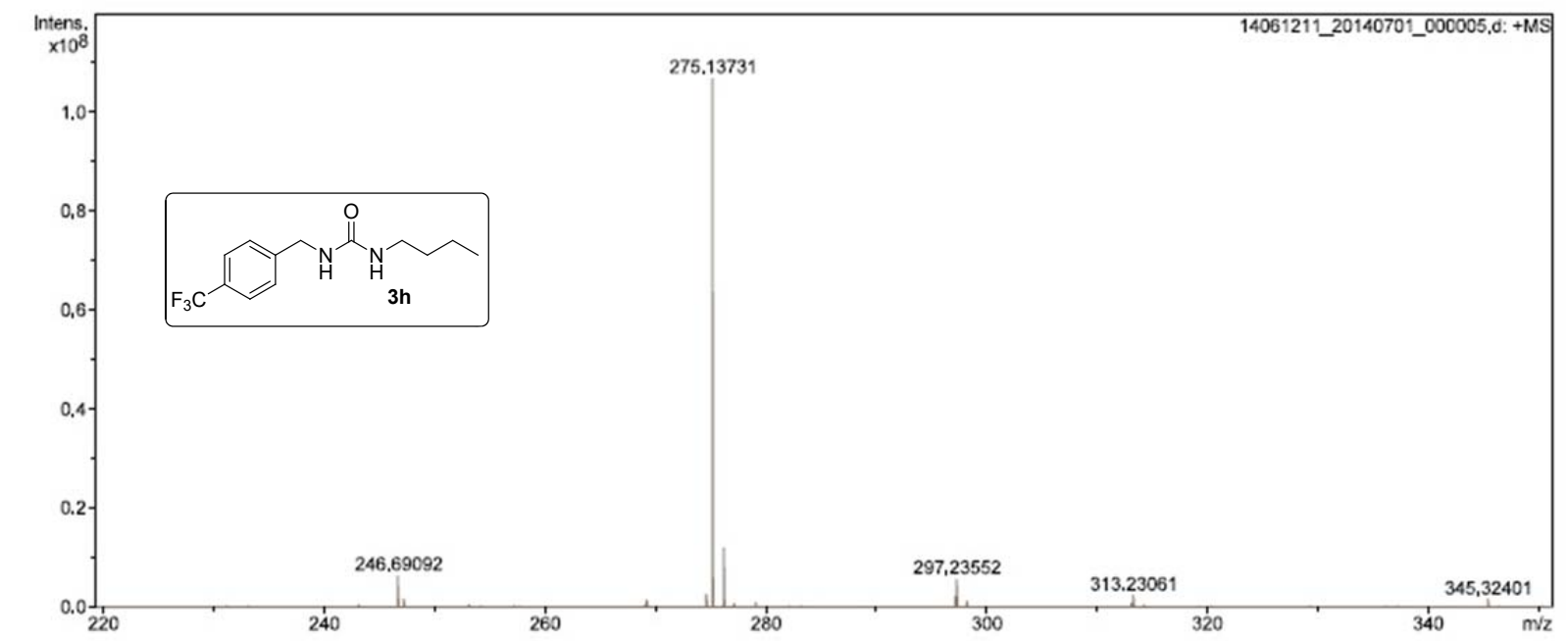

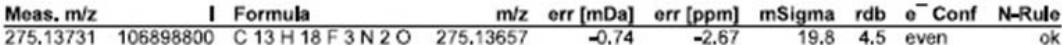



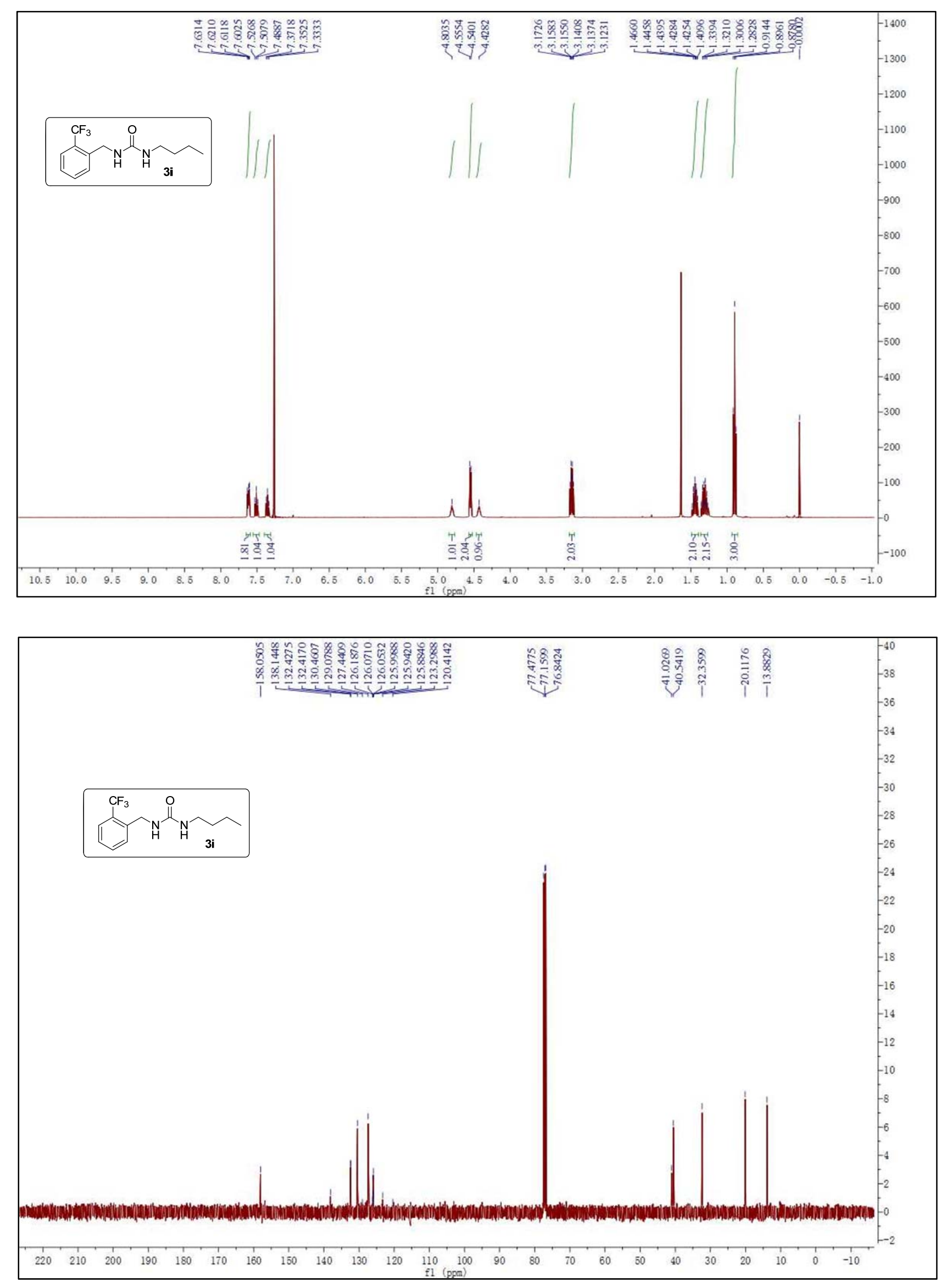

35 

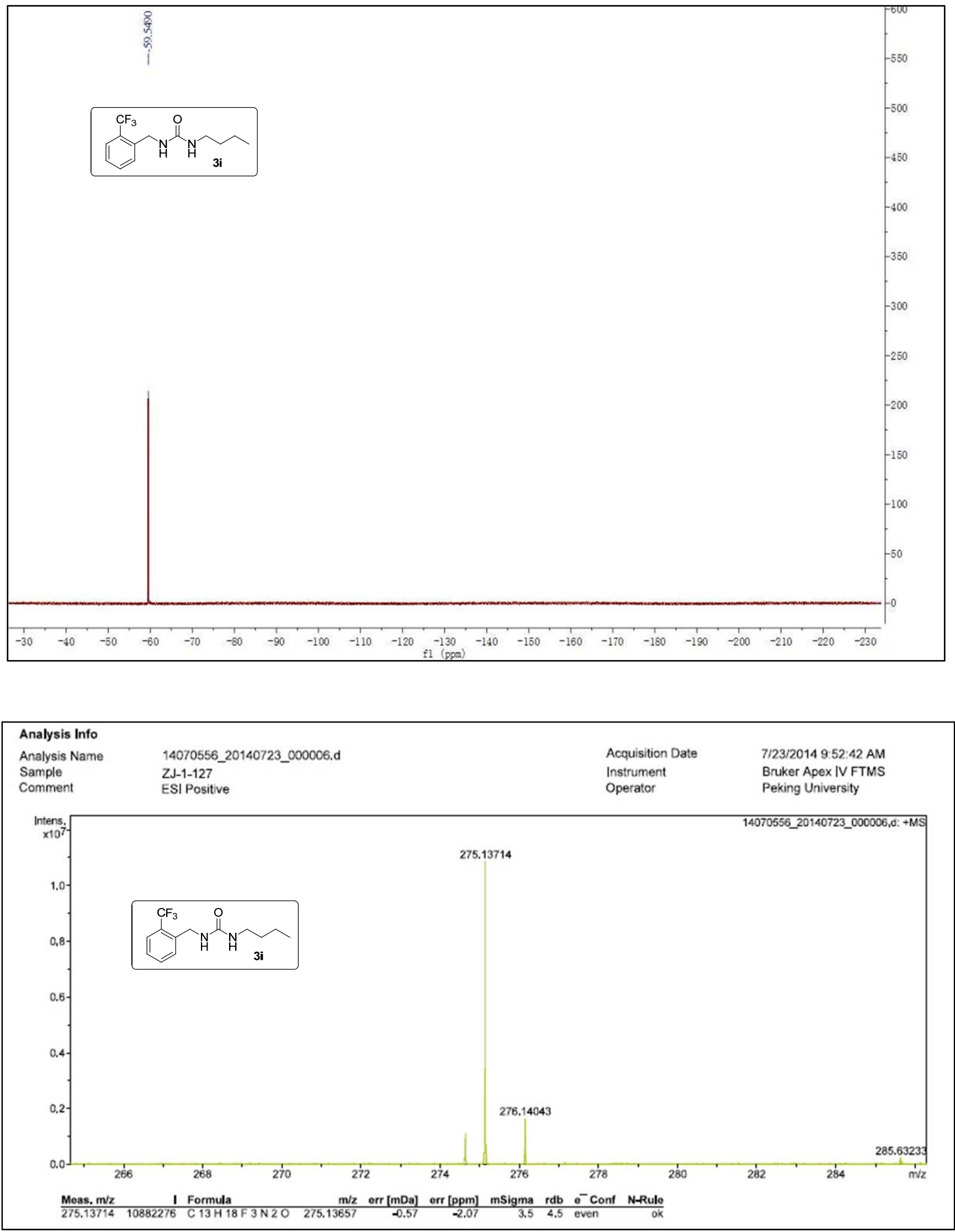

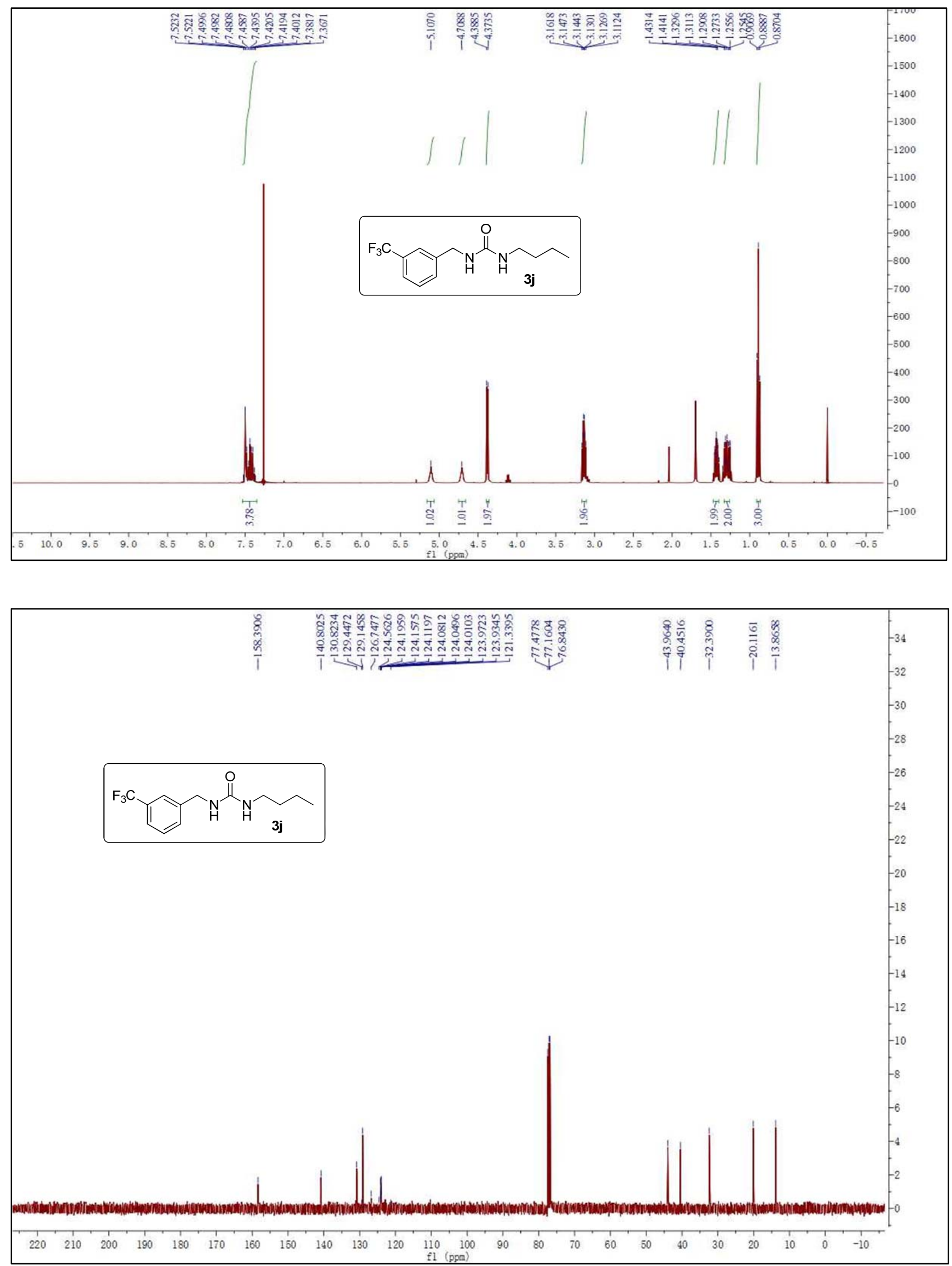

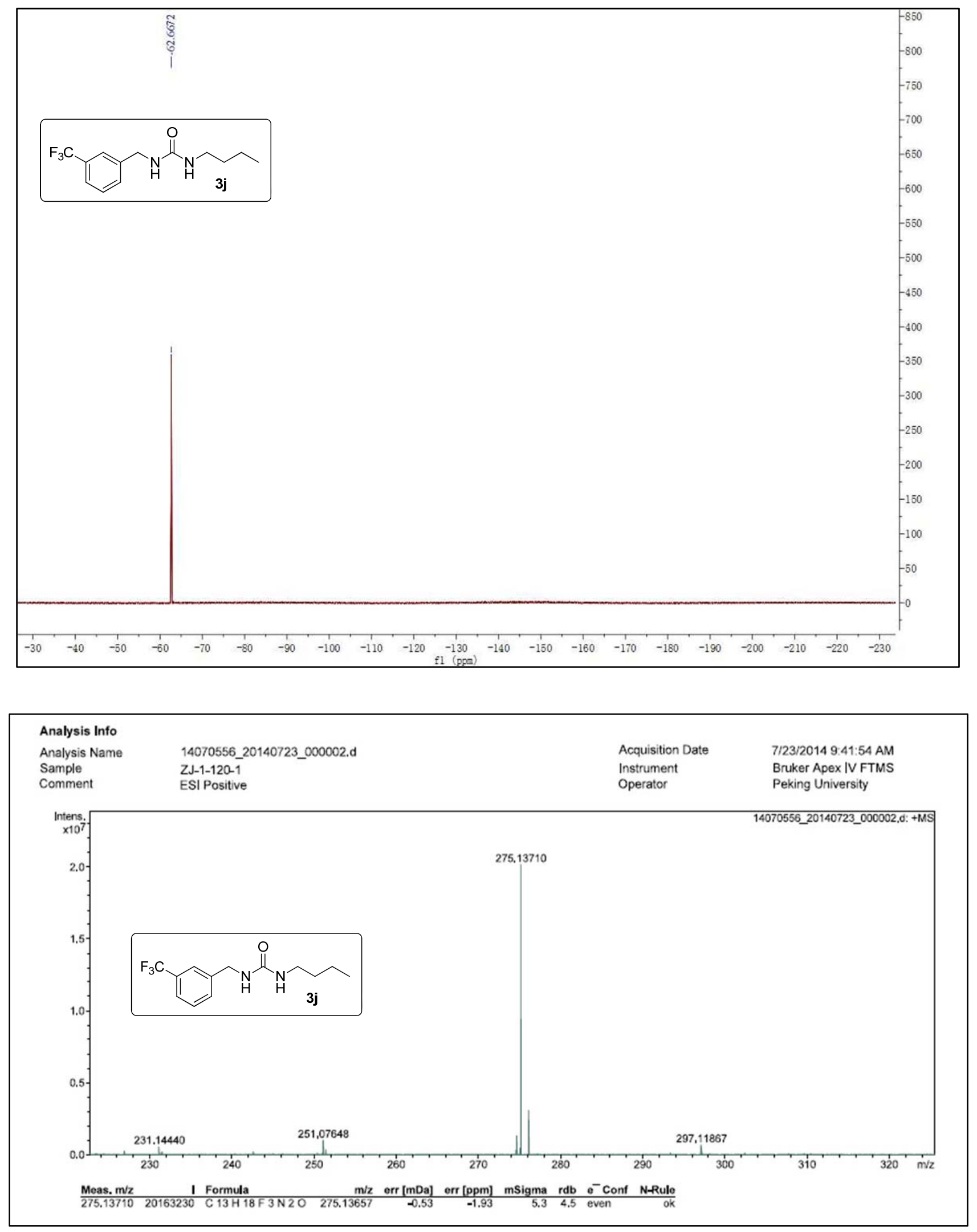

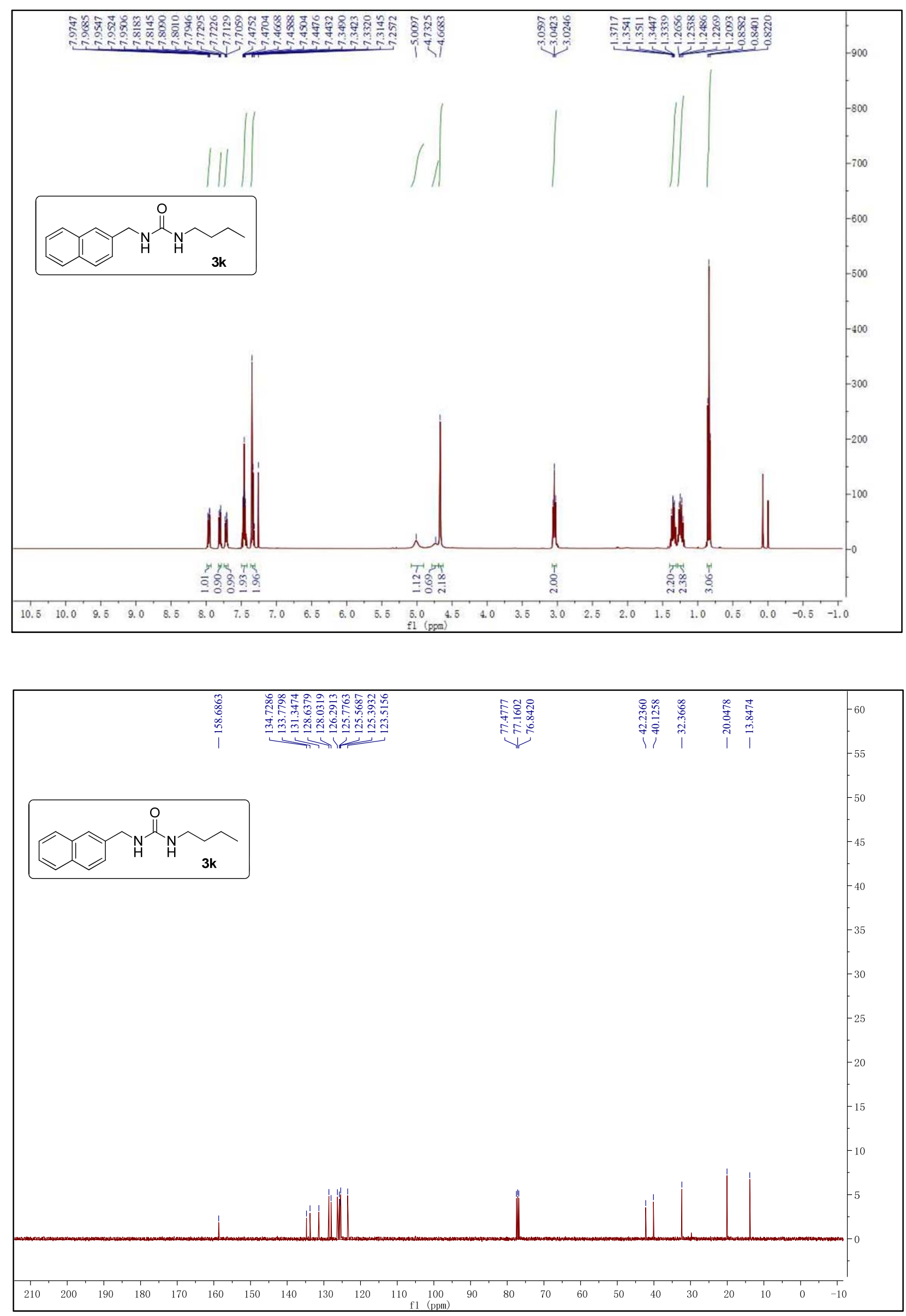

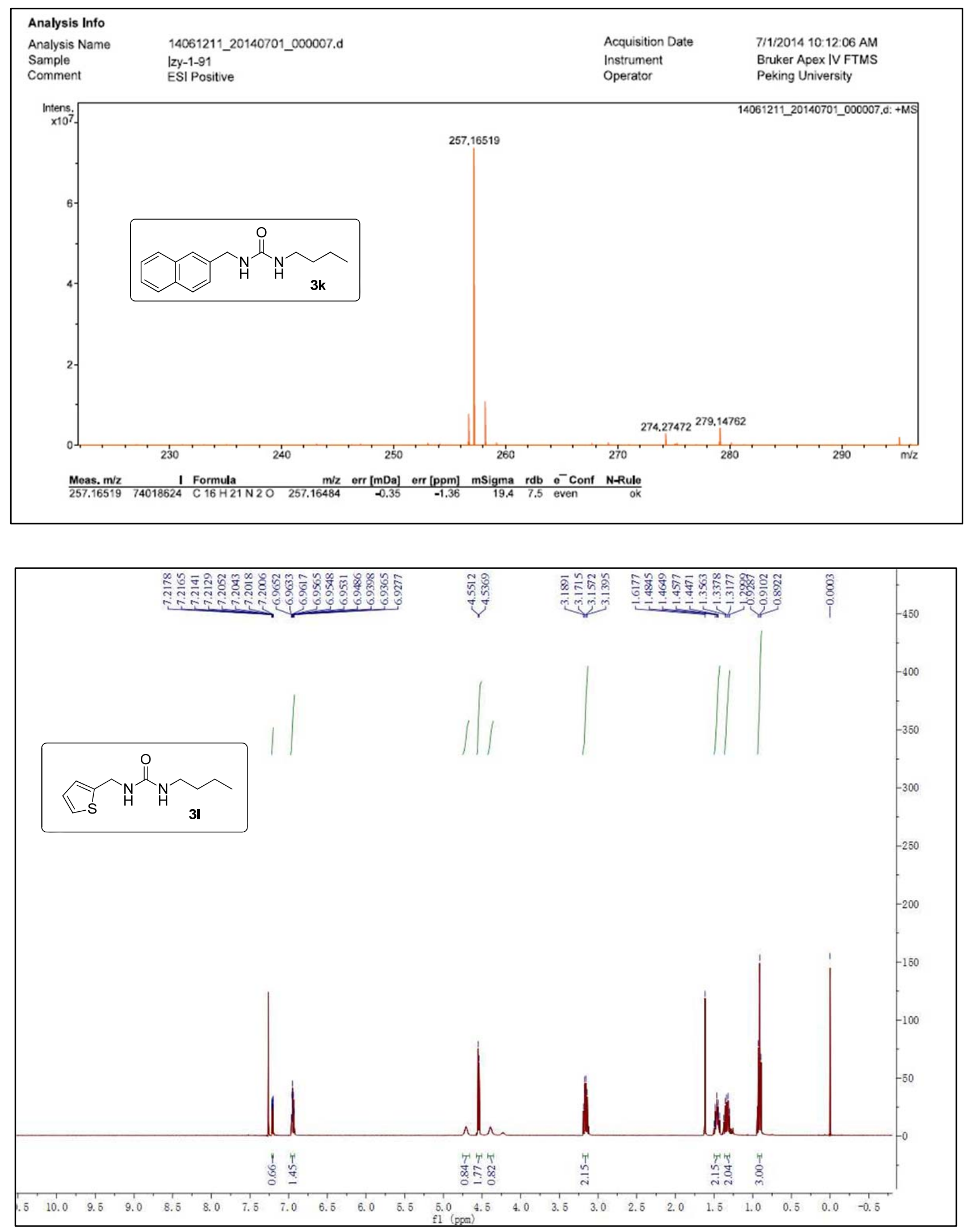

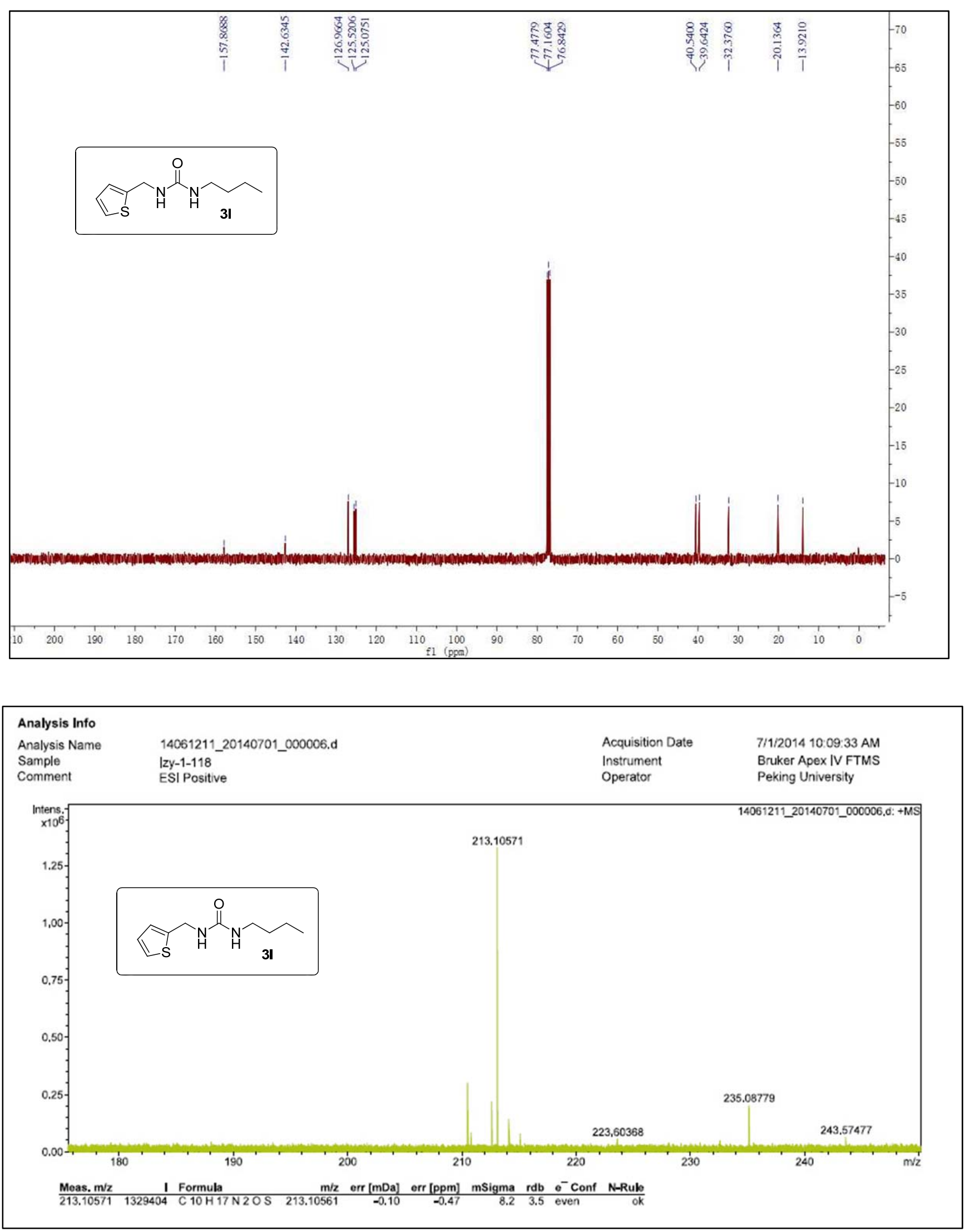

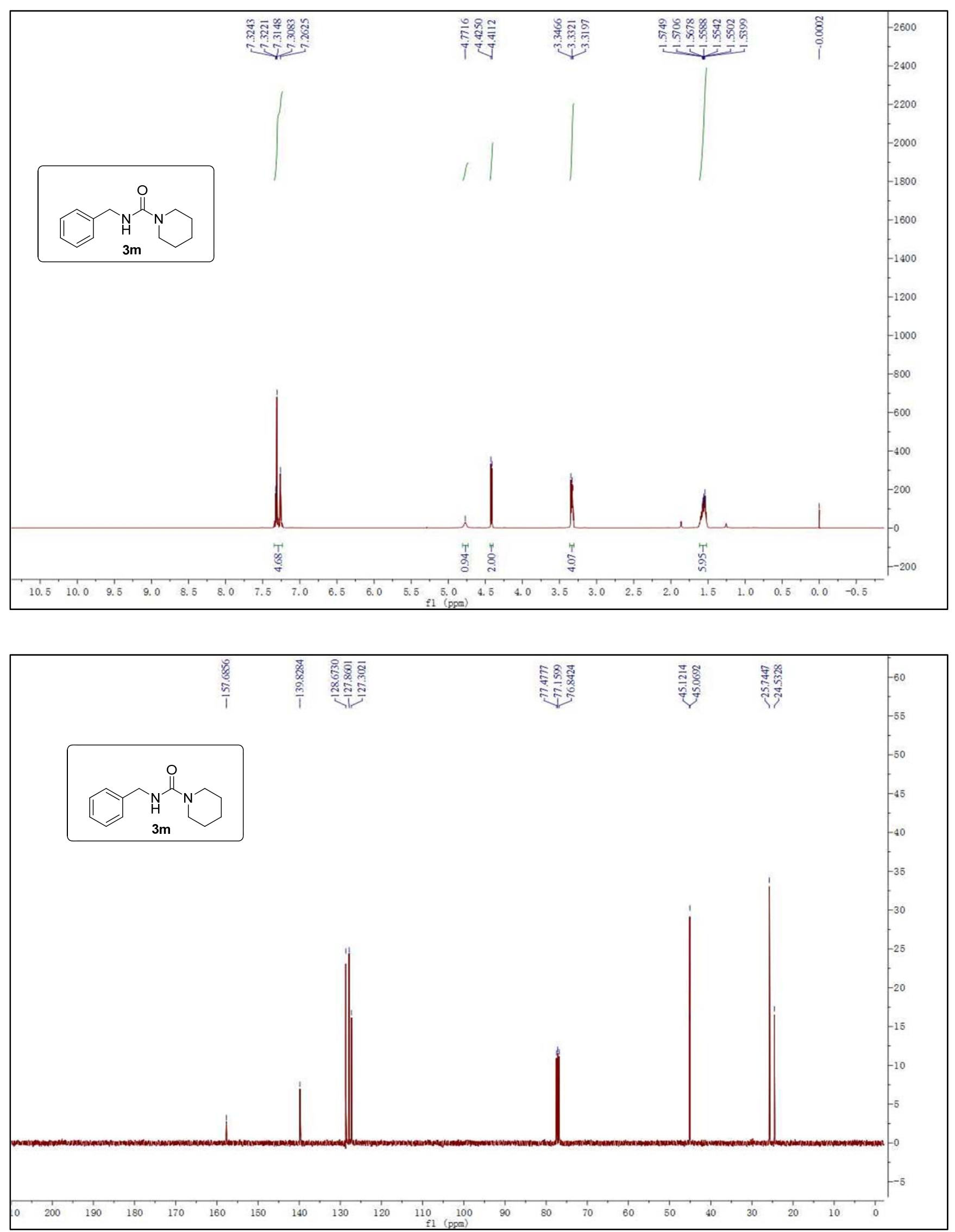

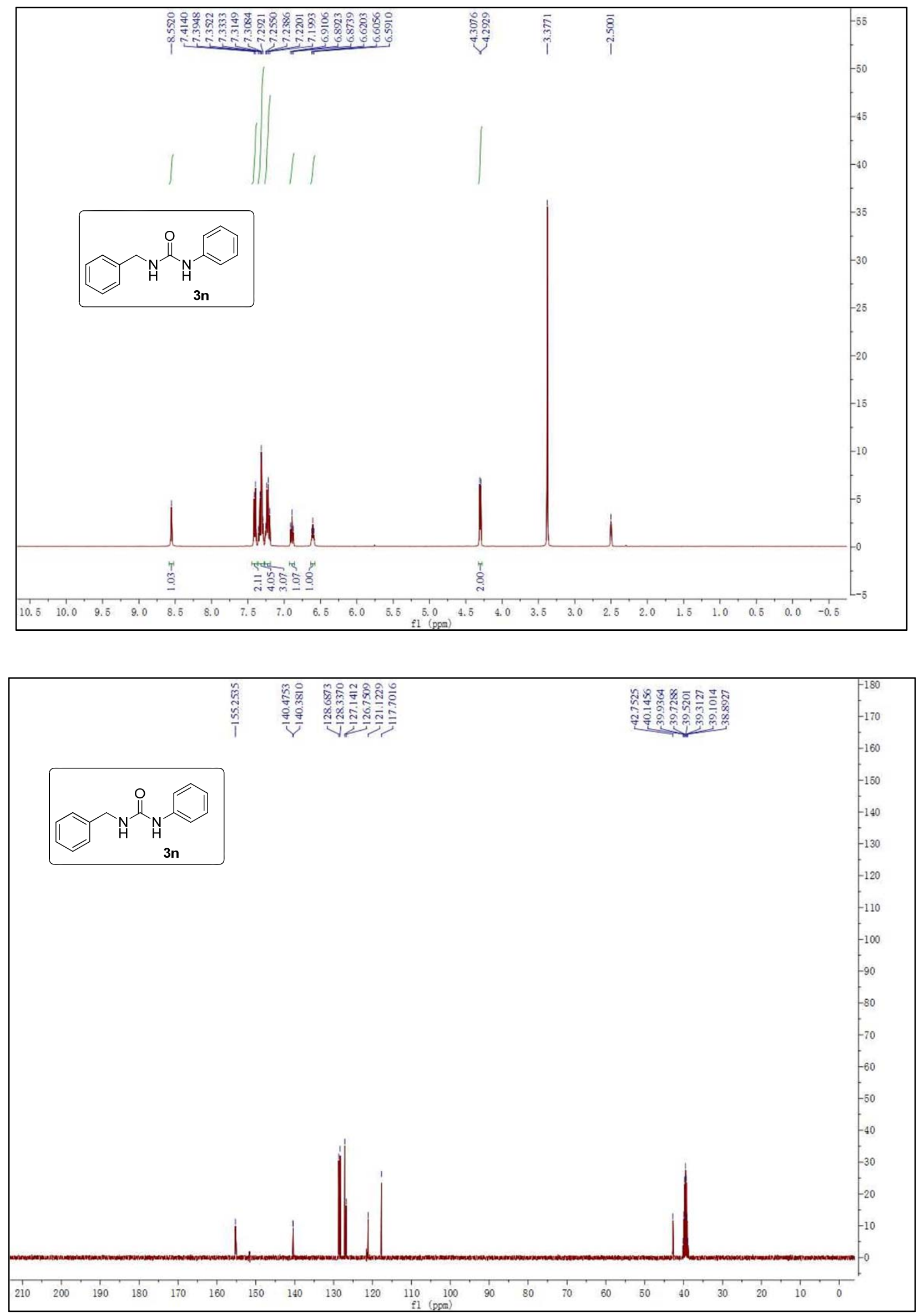

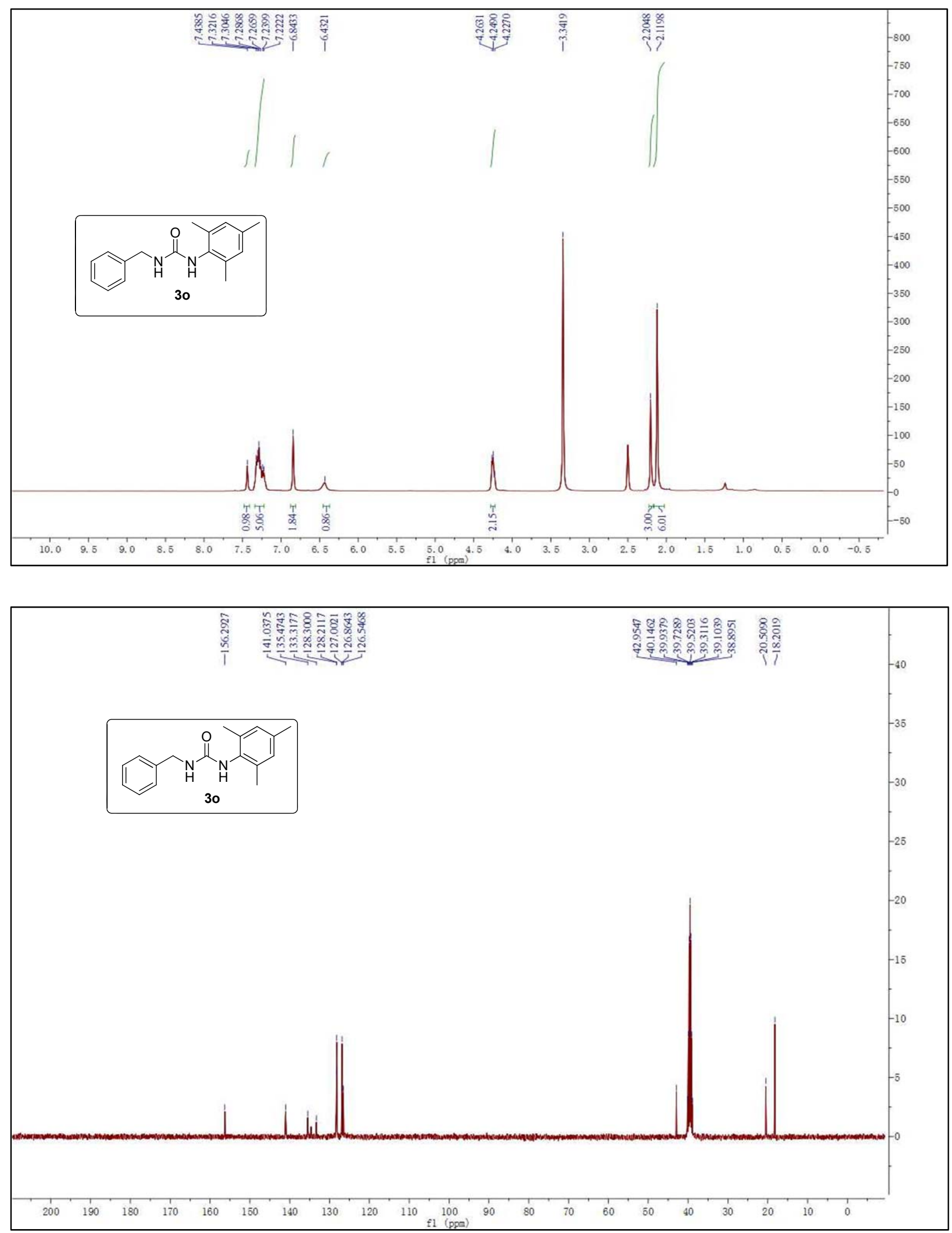

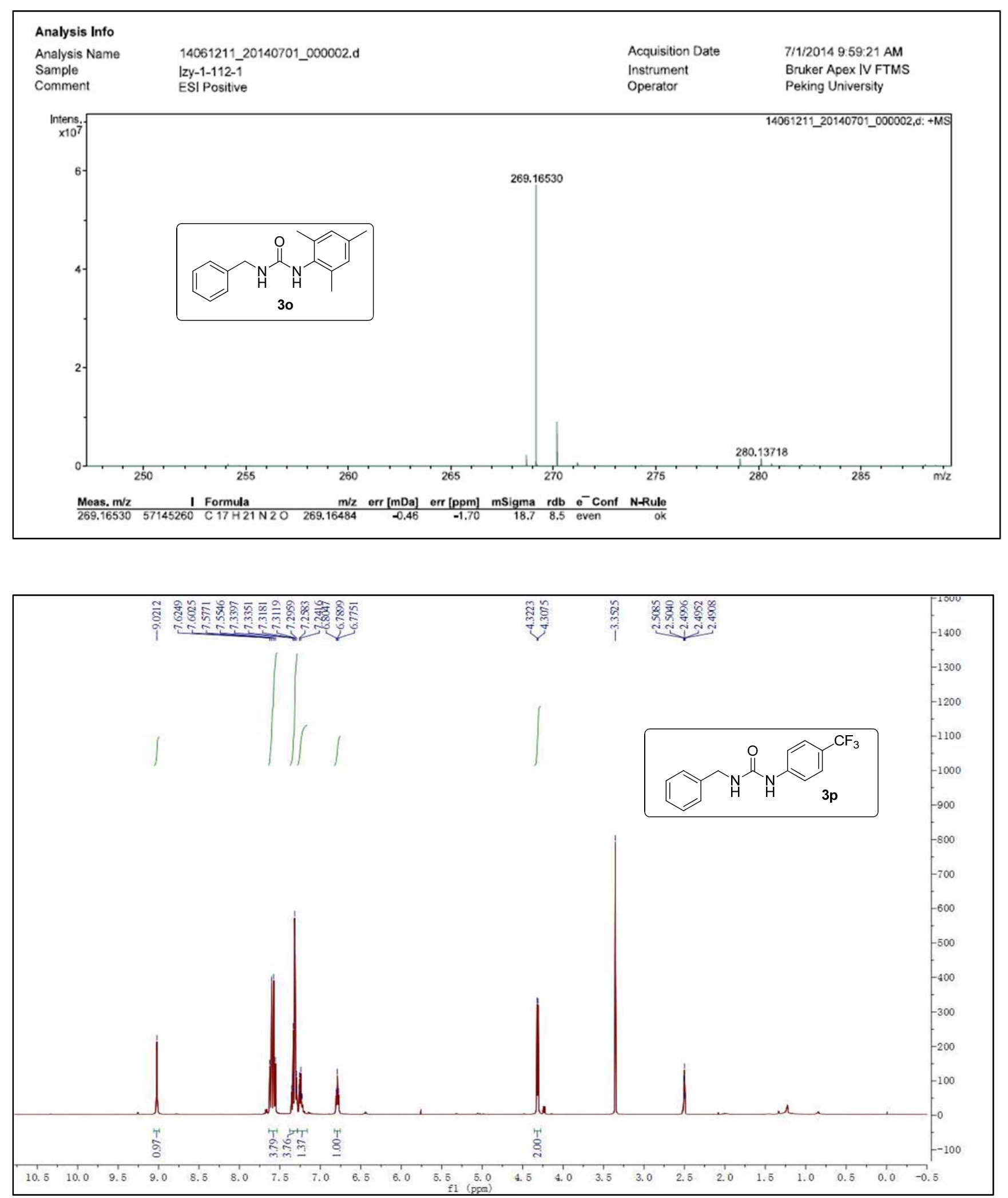

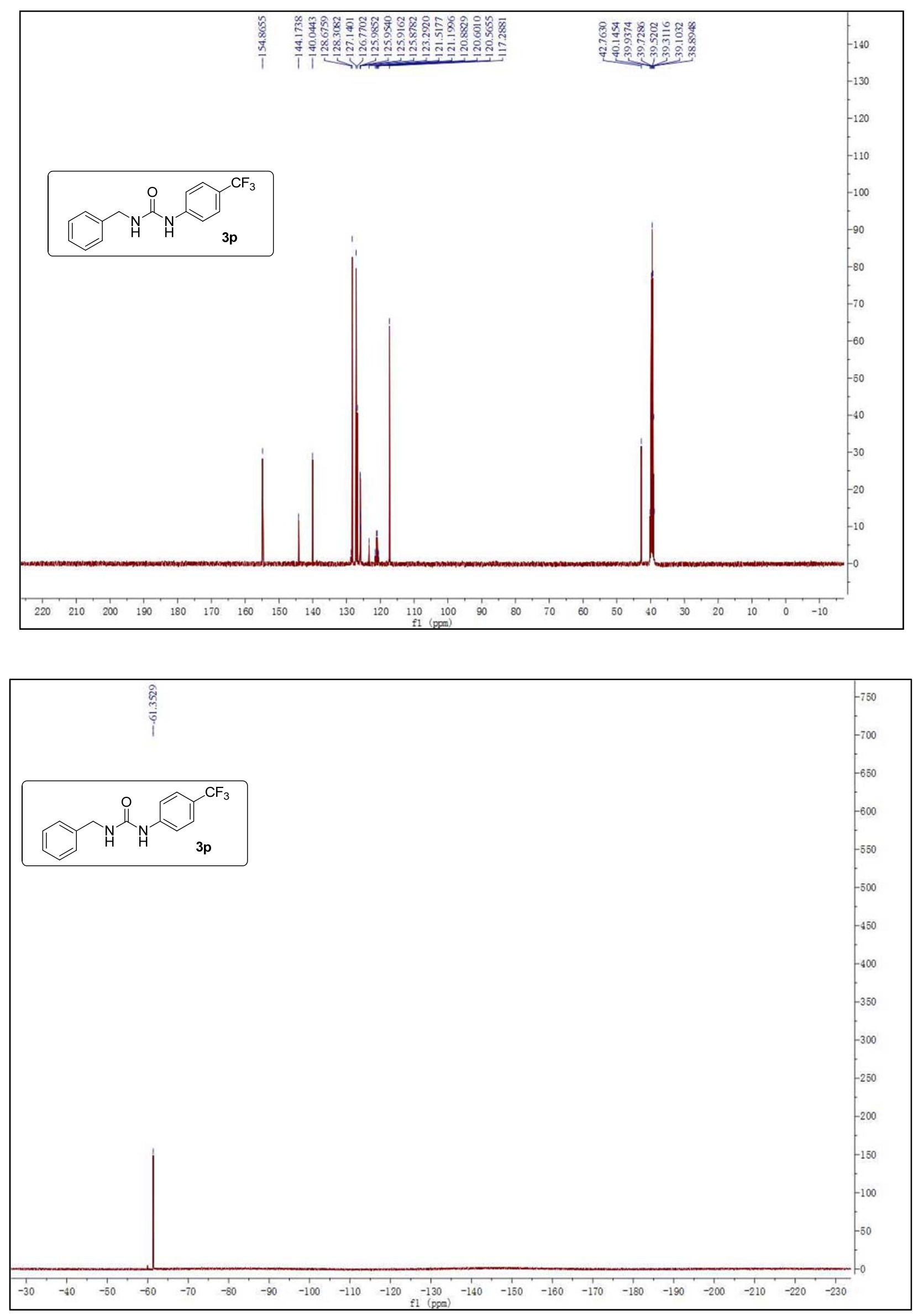

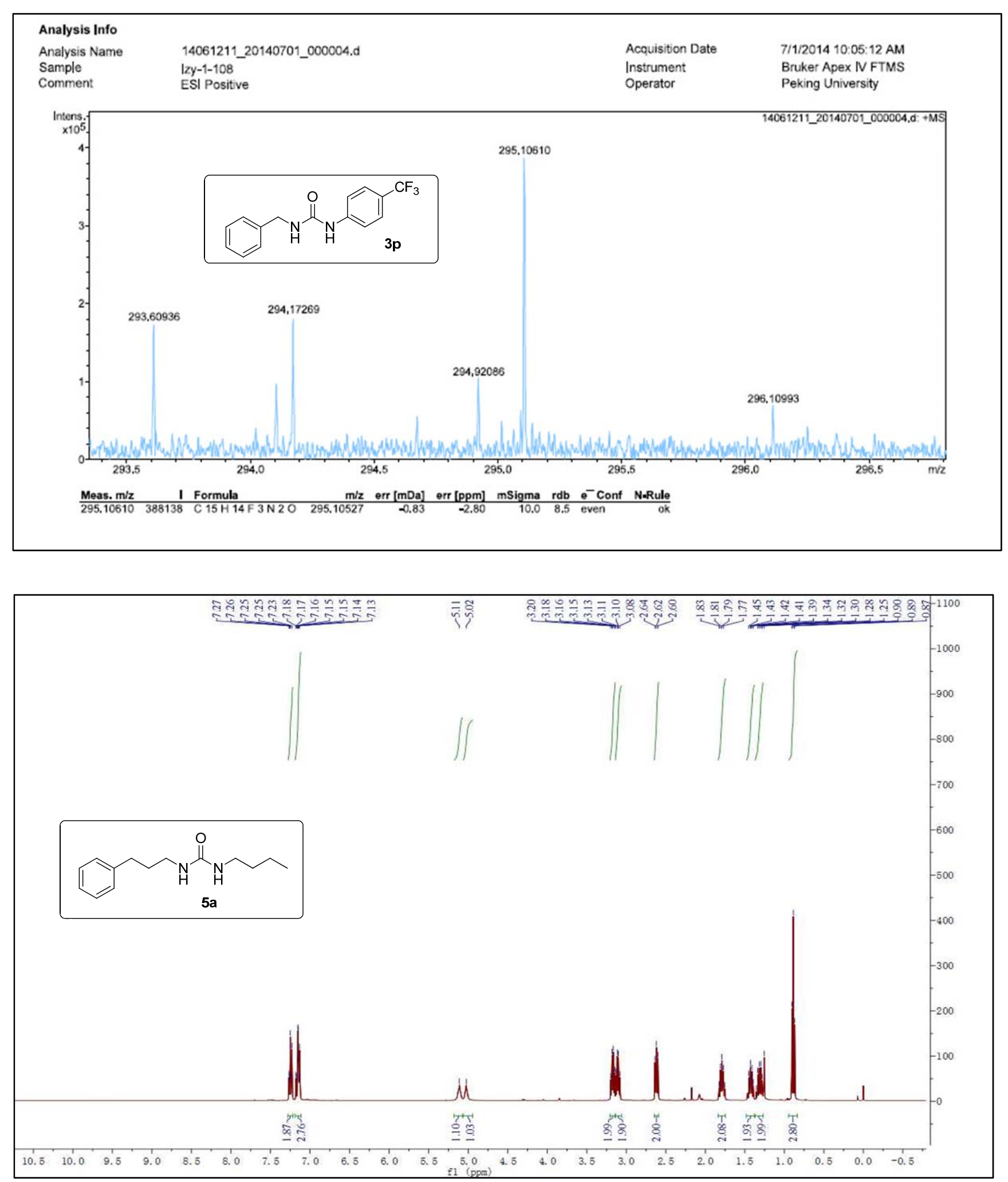

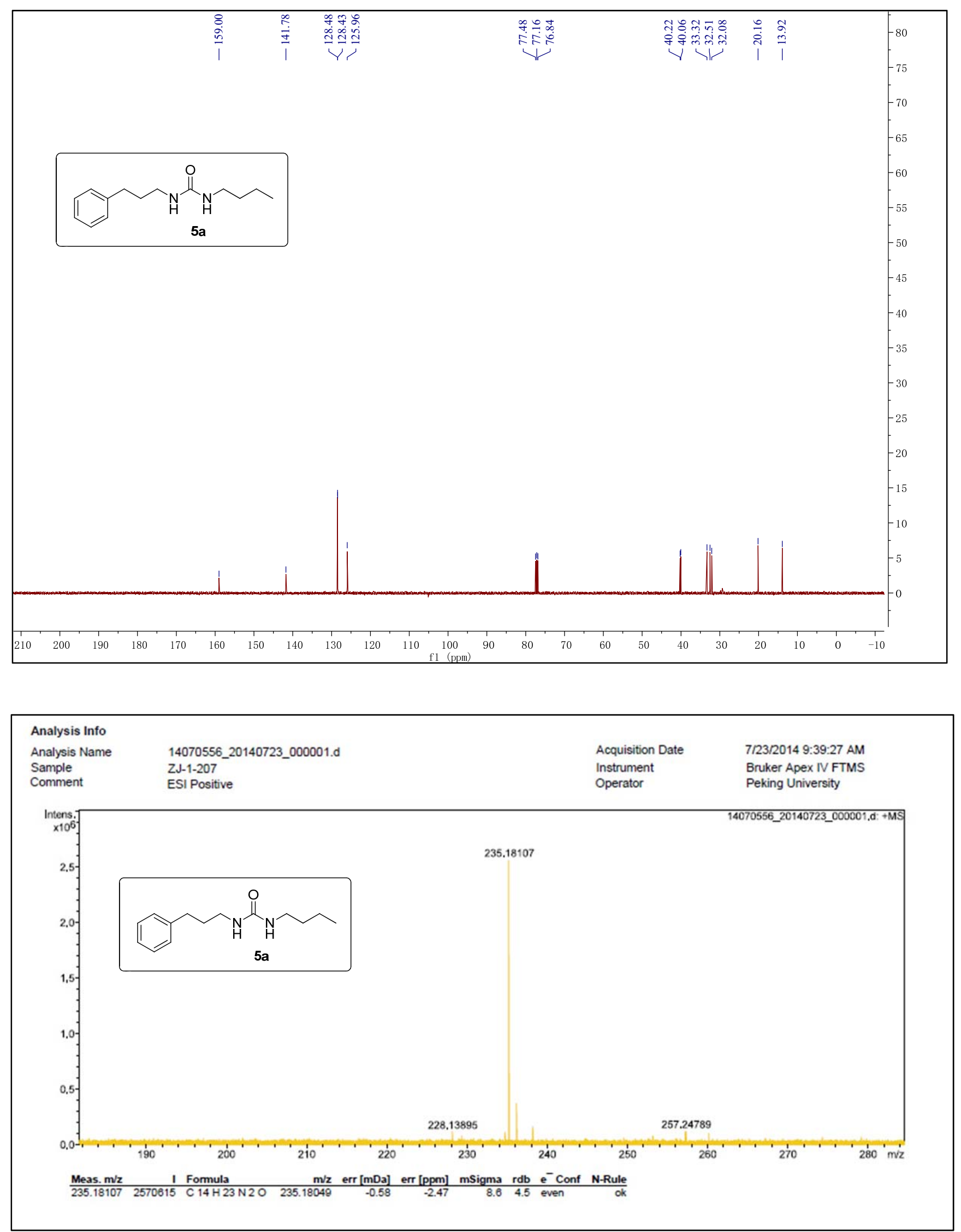

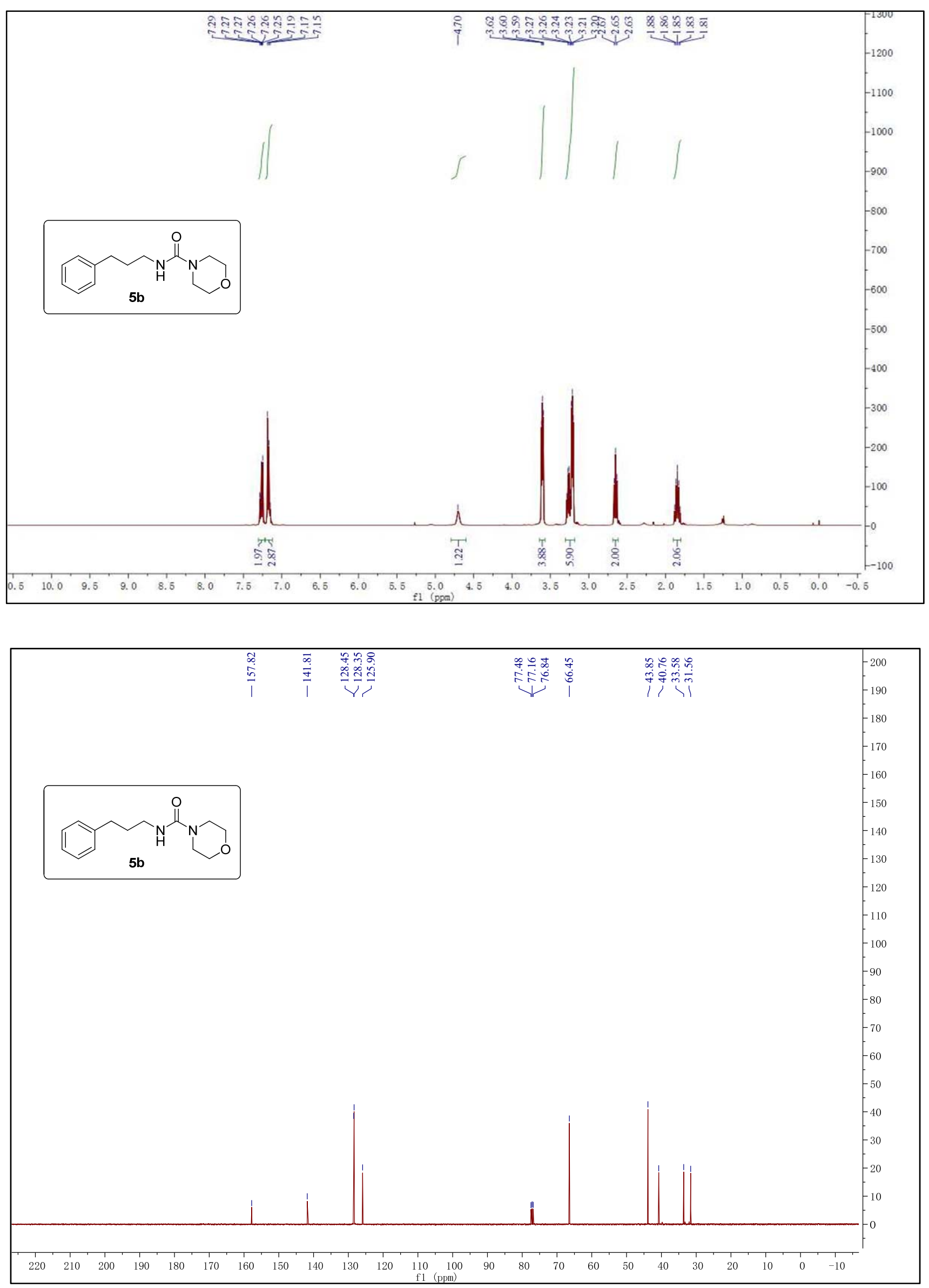

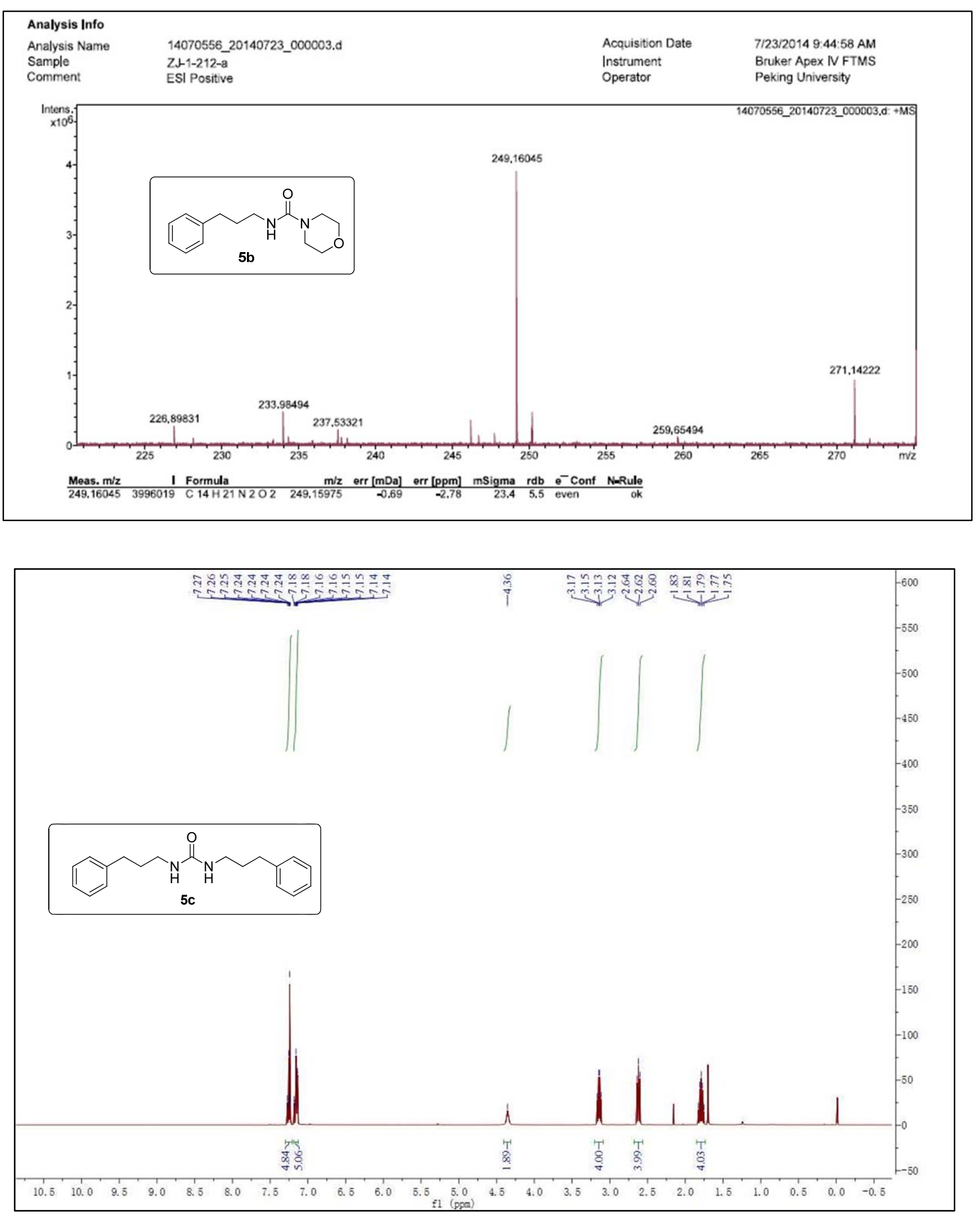

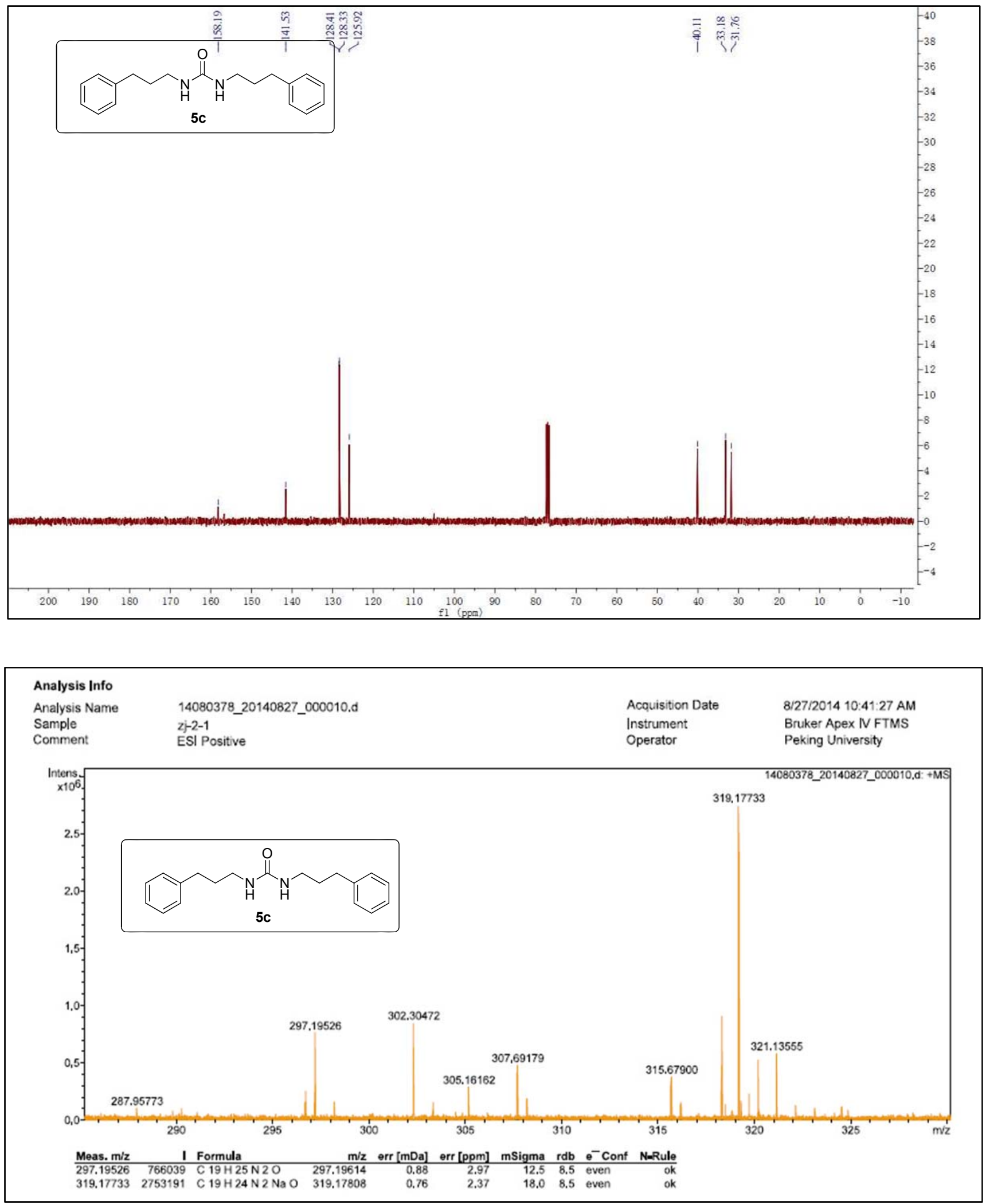

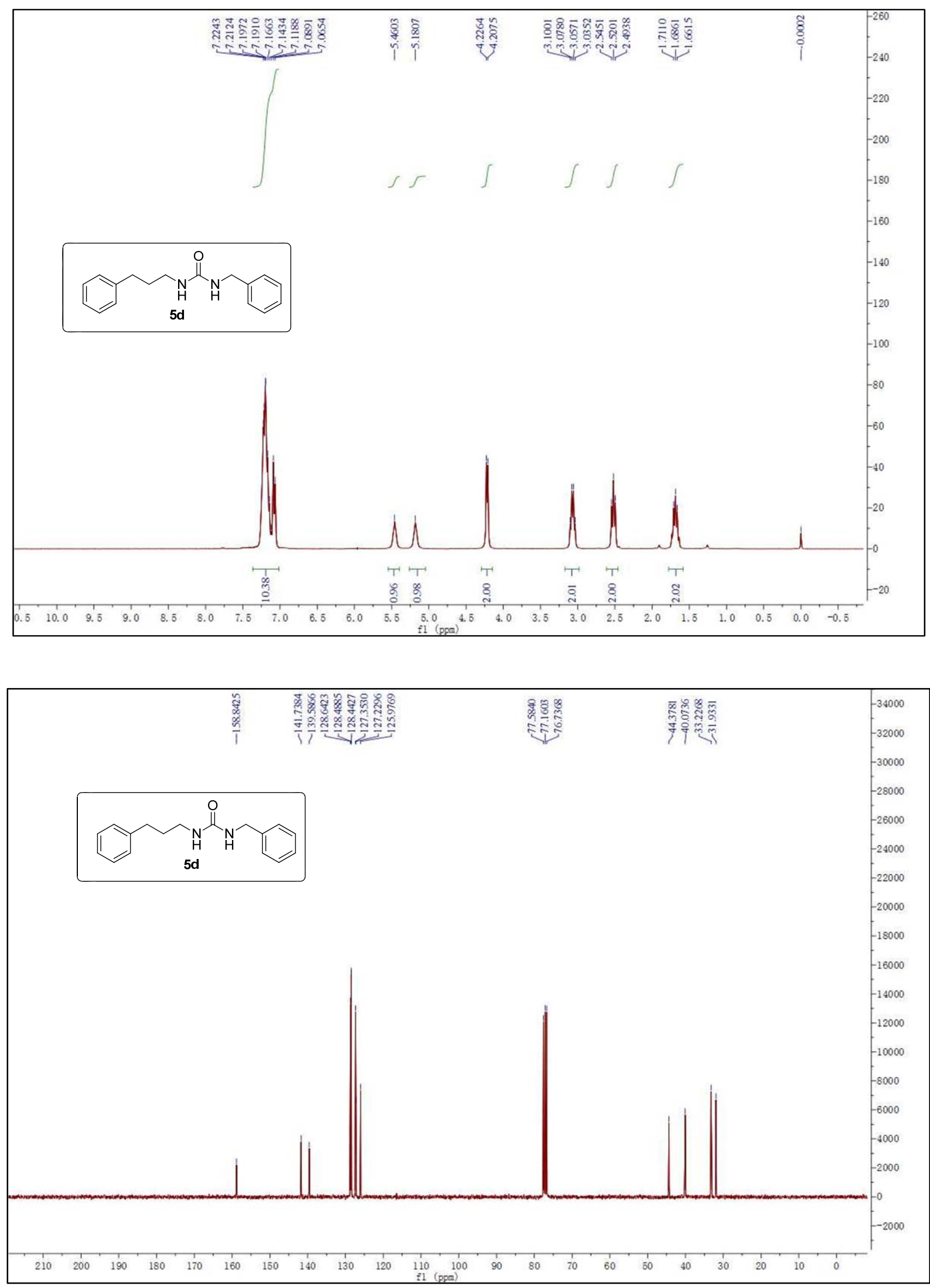

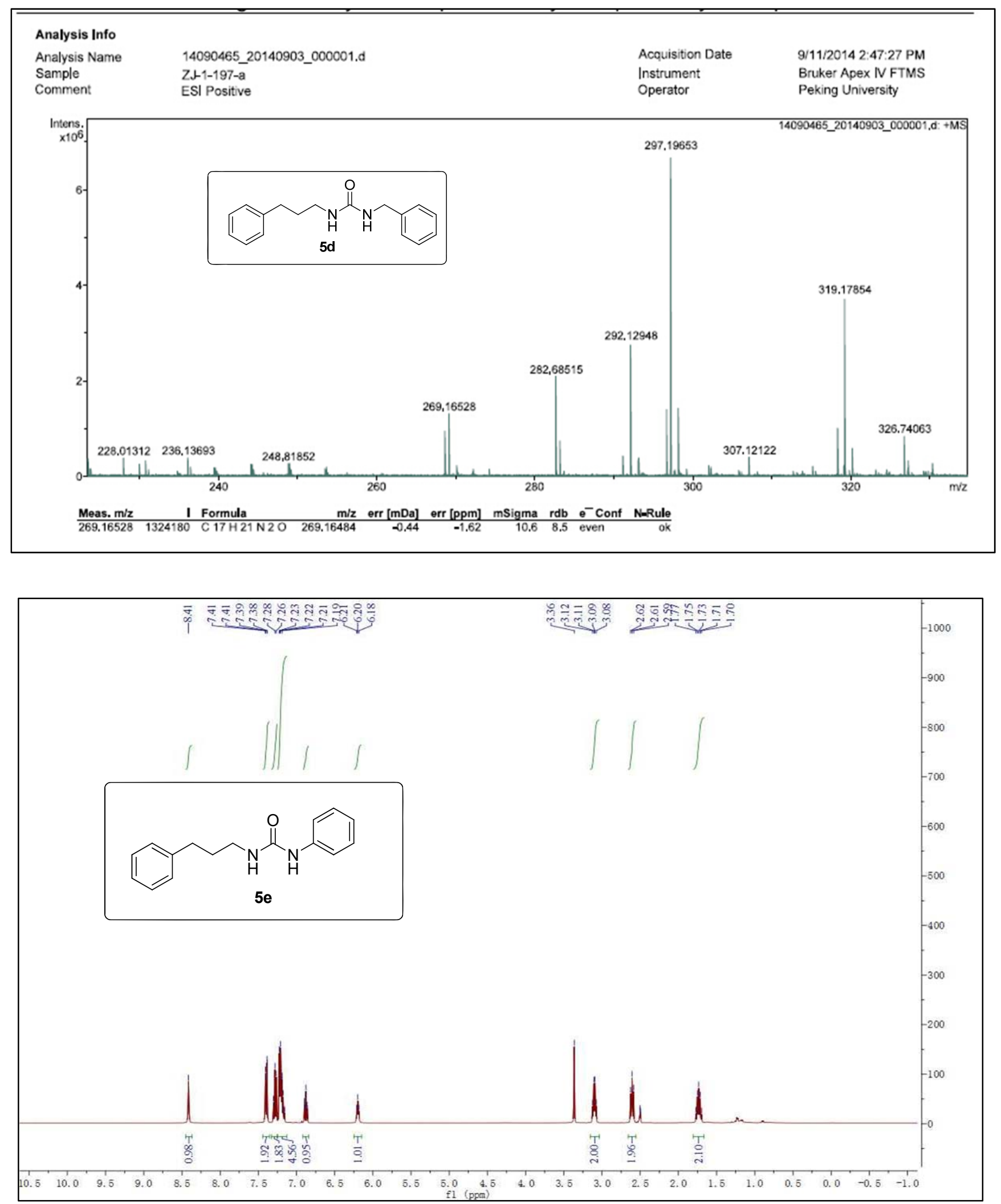

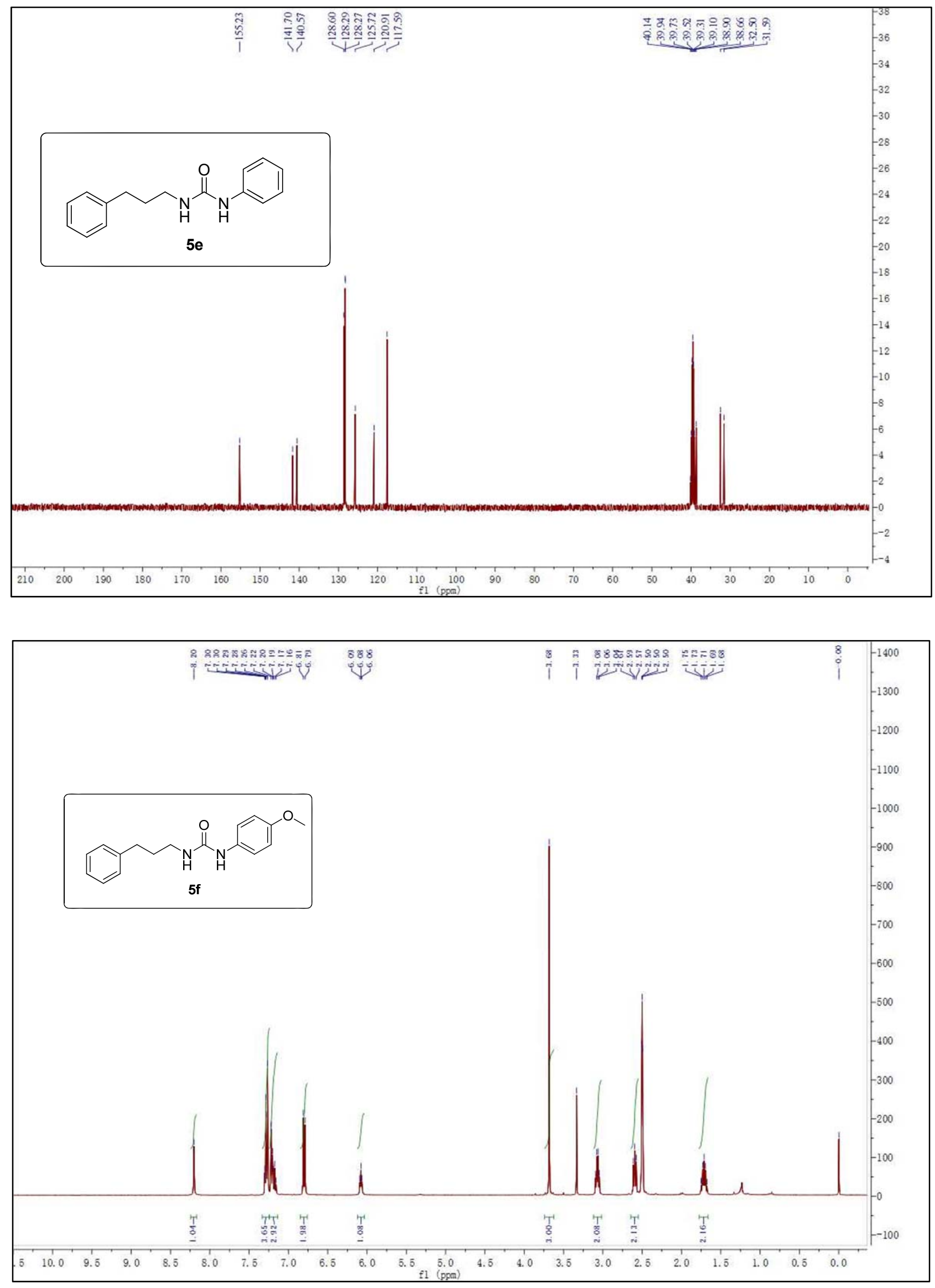

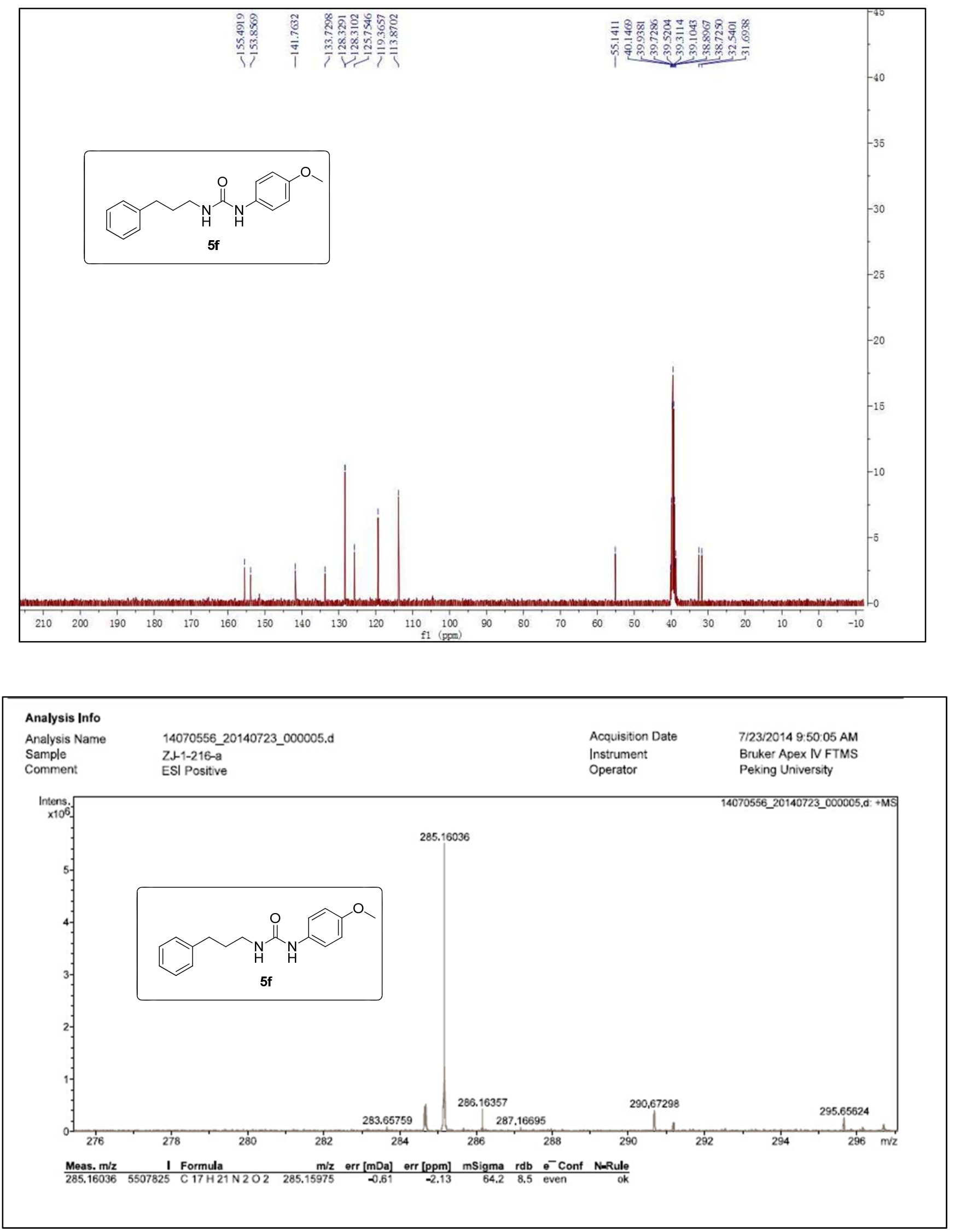

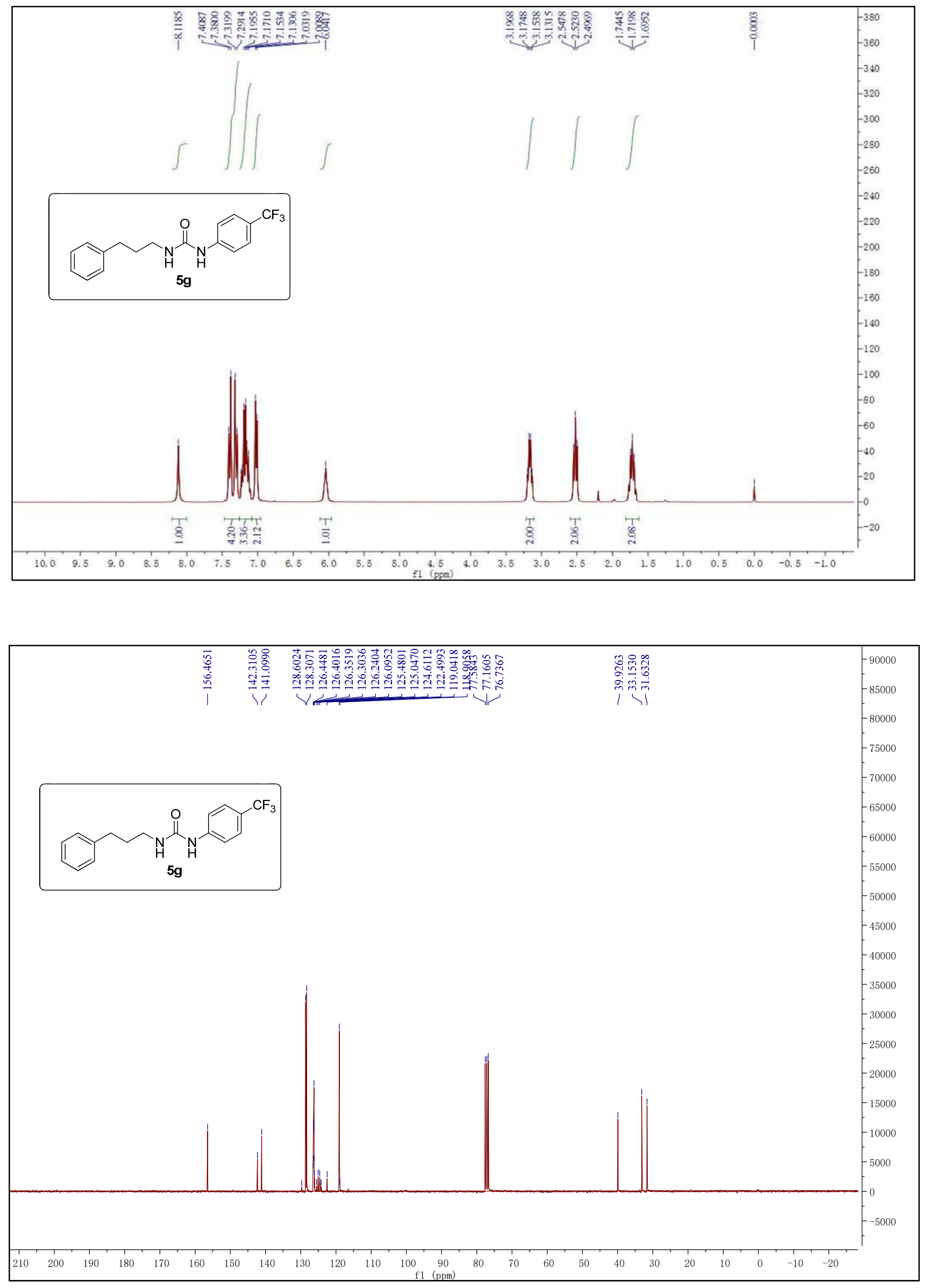

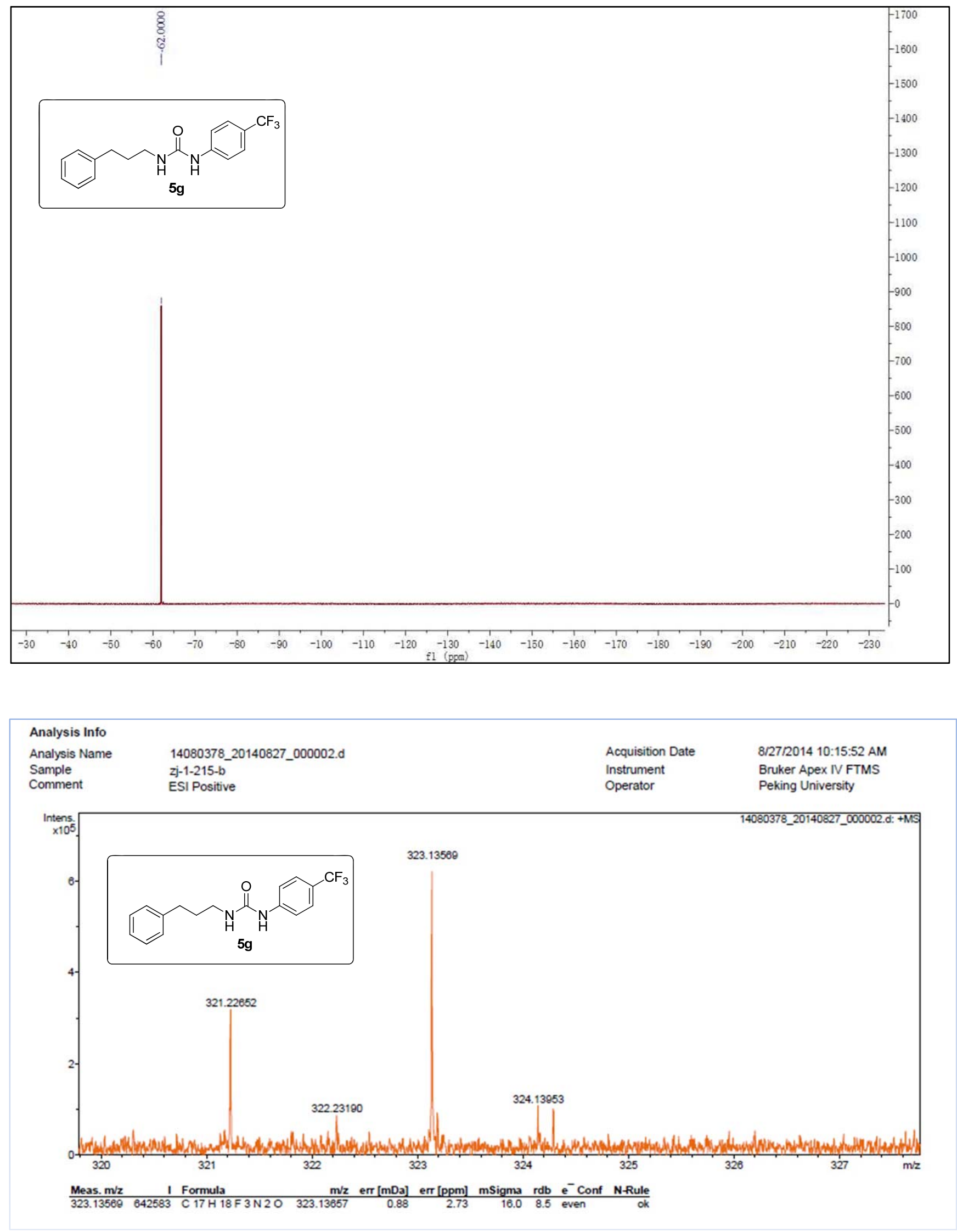

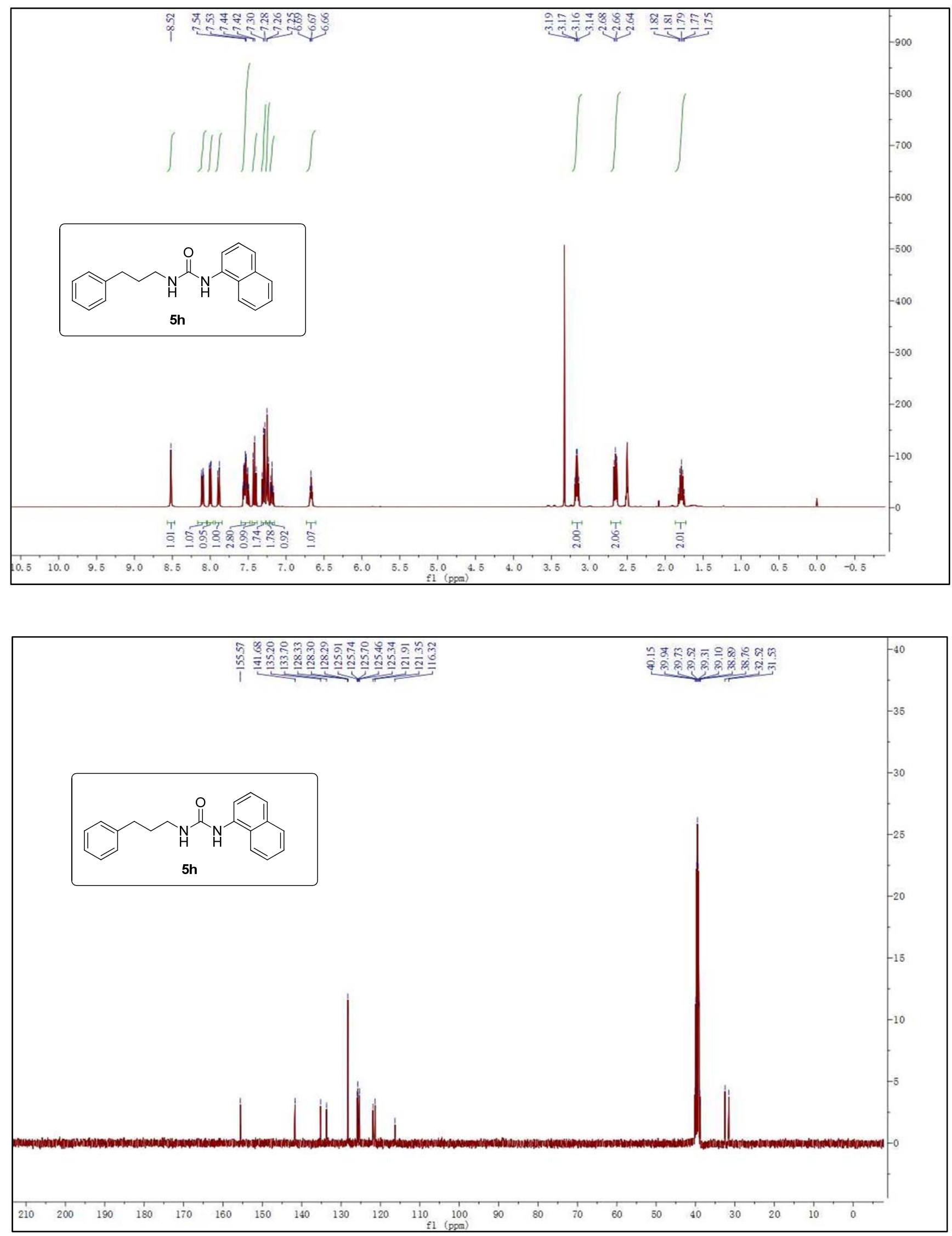

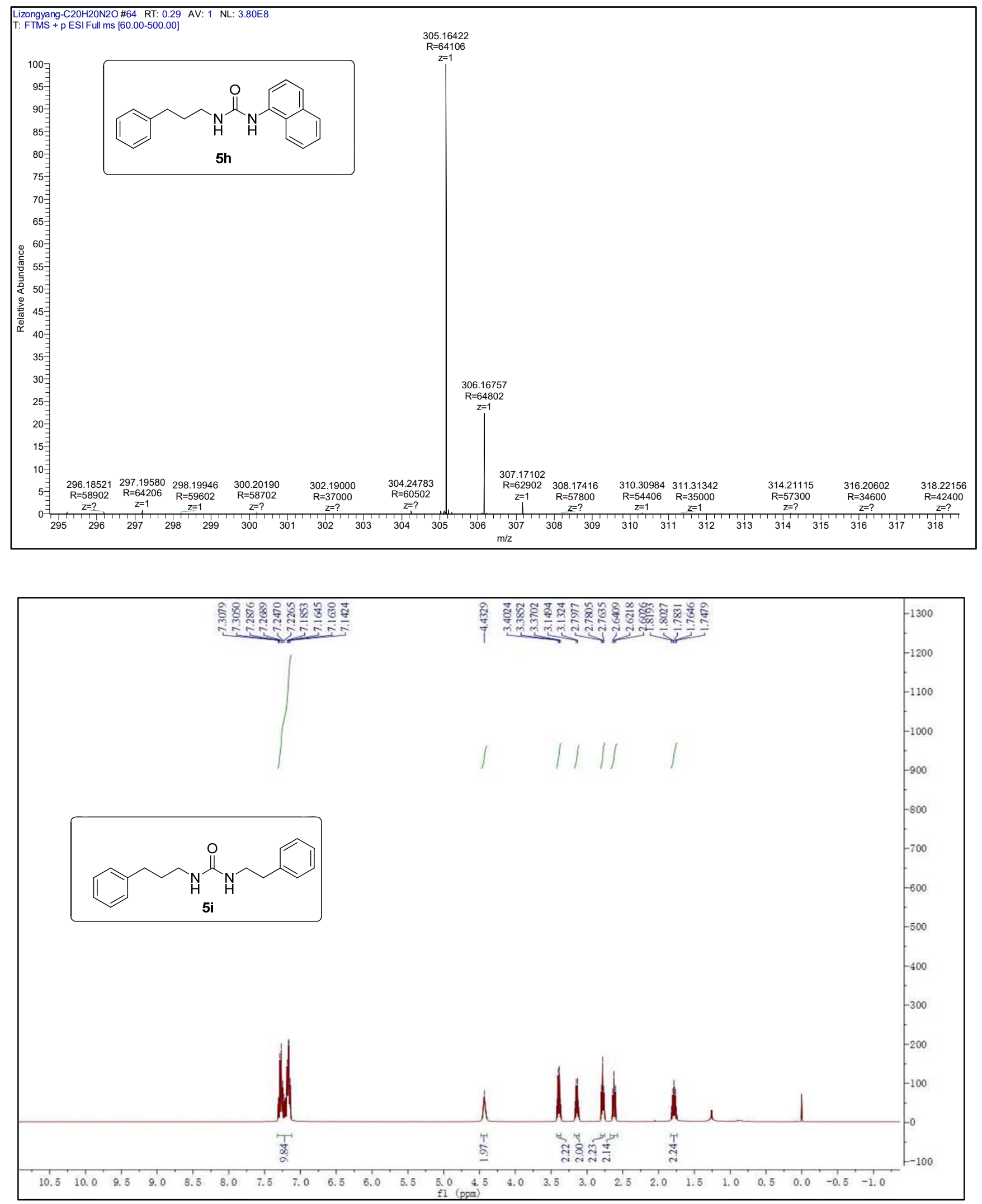


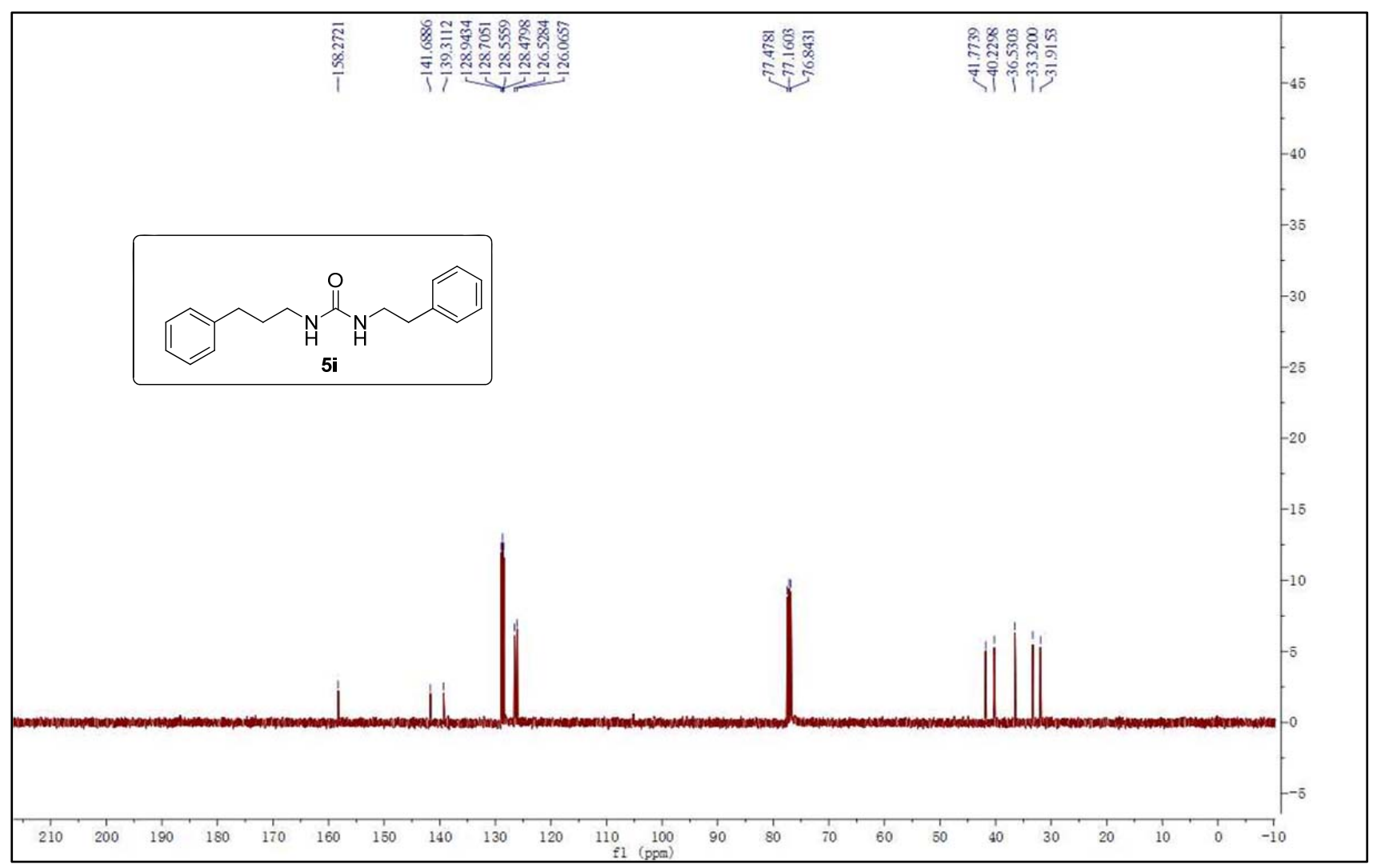

\section{Analysis Info}

Analysis Name

Sample

14080378_20140827_000008.d

zj-1-228

Acquisition Date

Instrument

8/27/2014 10:36:40 AM

Commen:

ESI Positive

Operator

Bruker Apex IV FTMS

Peking University

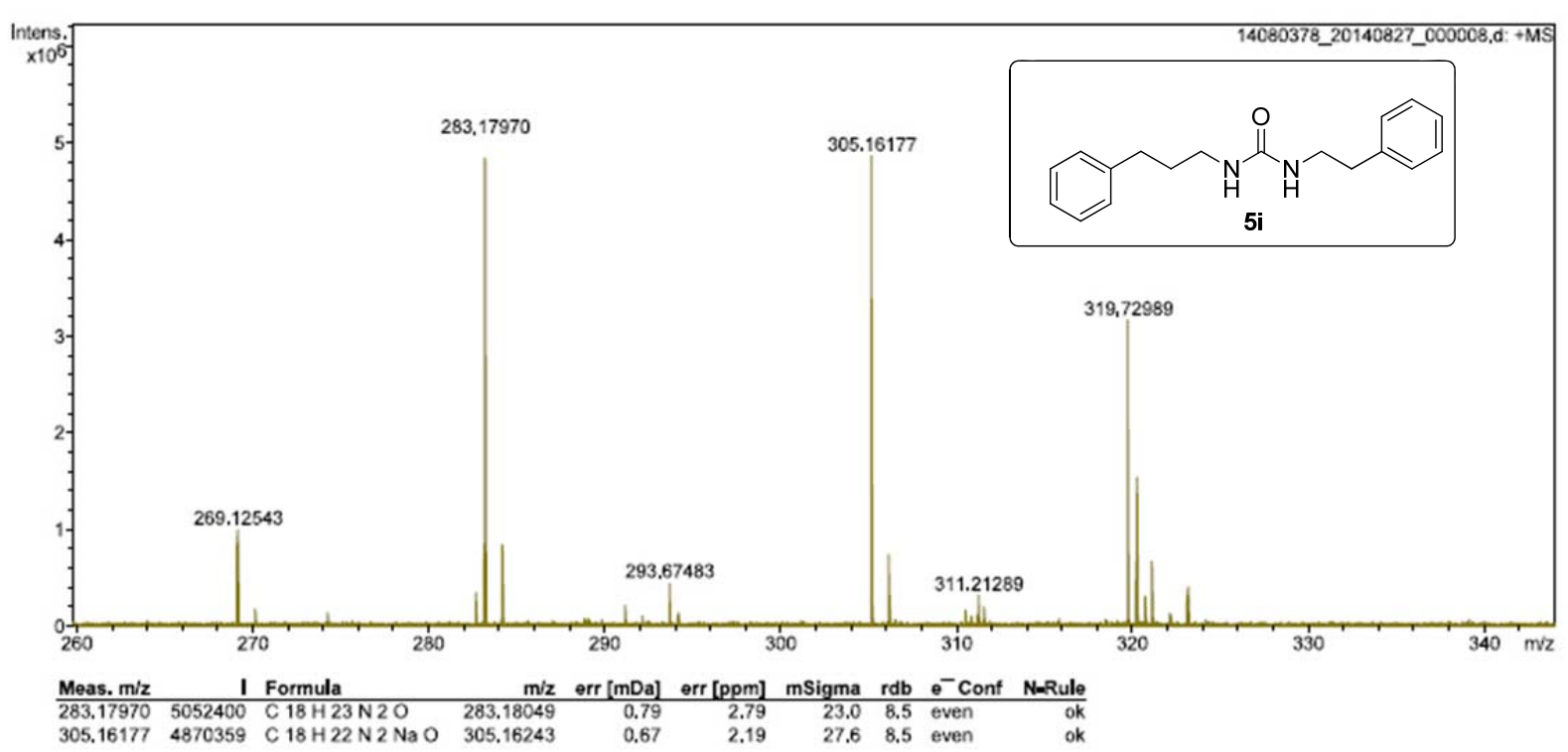



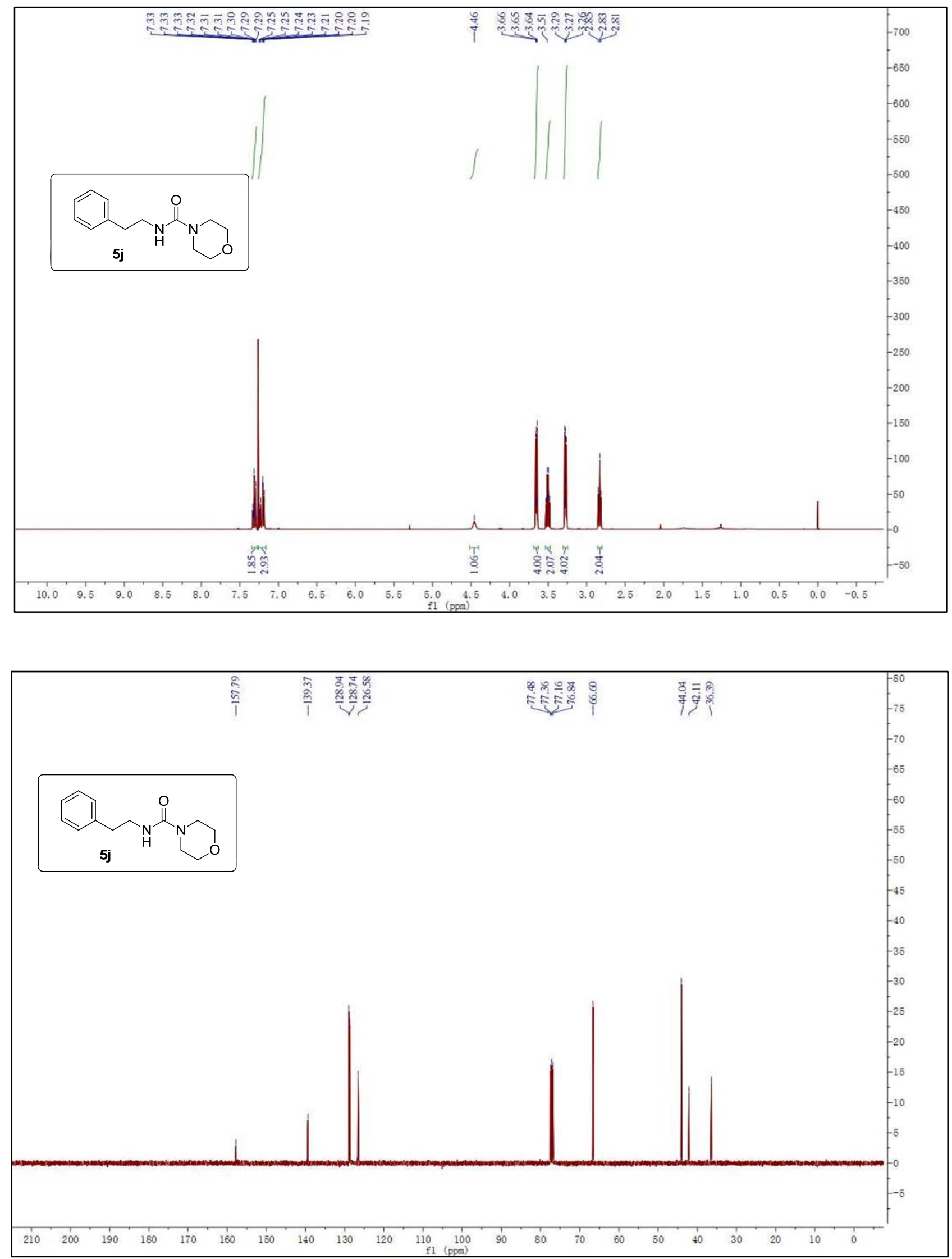

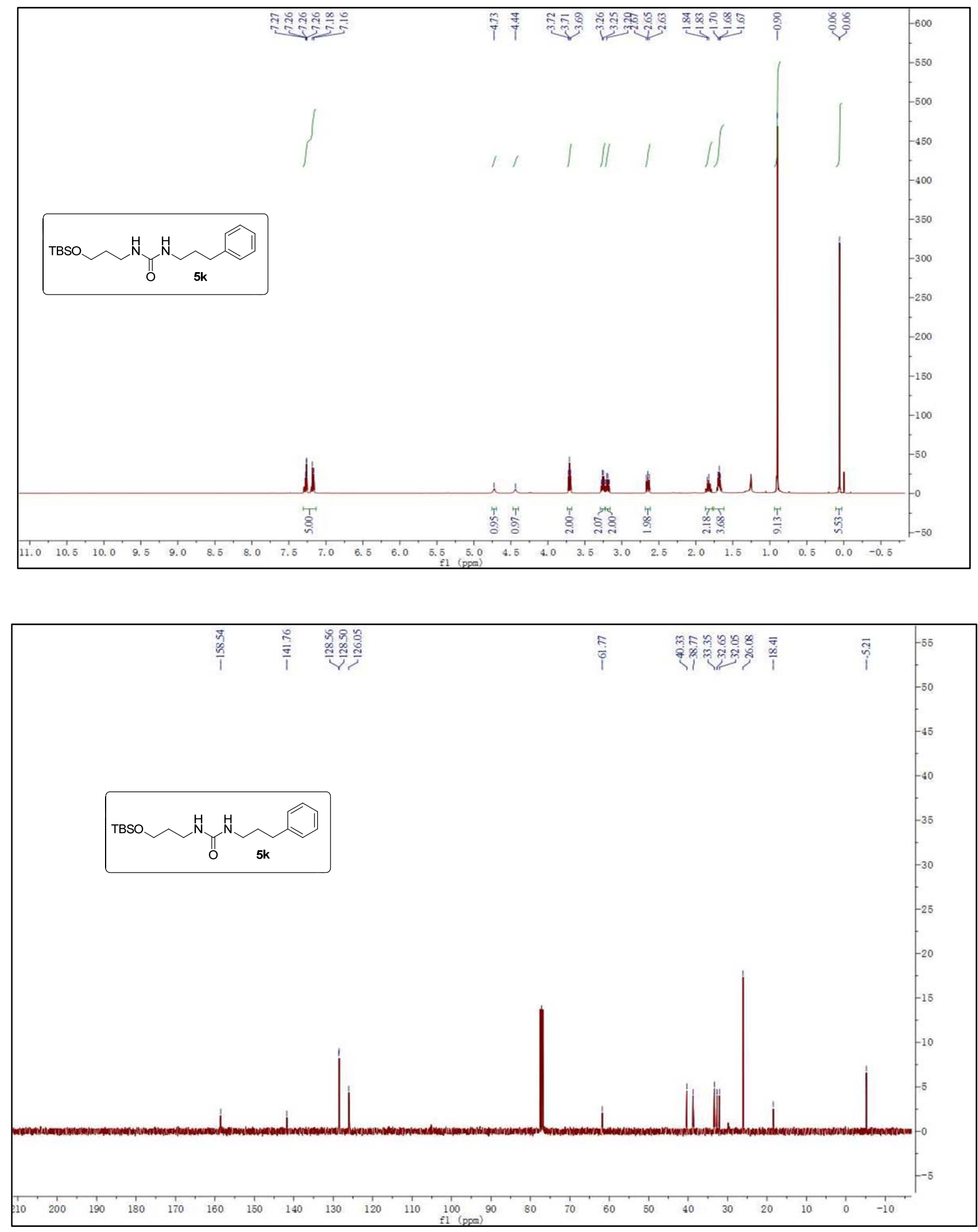

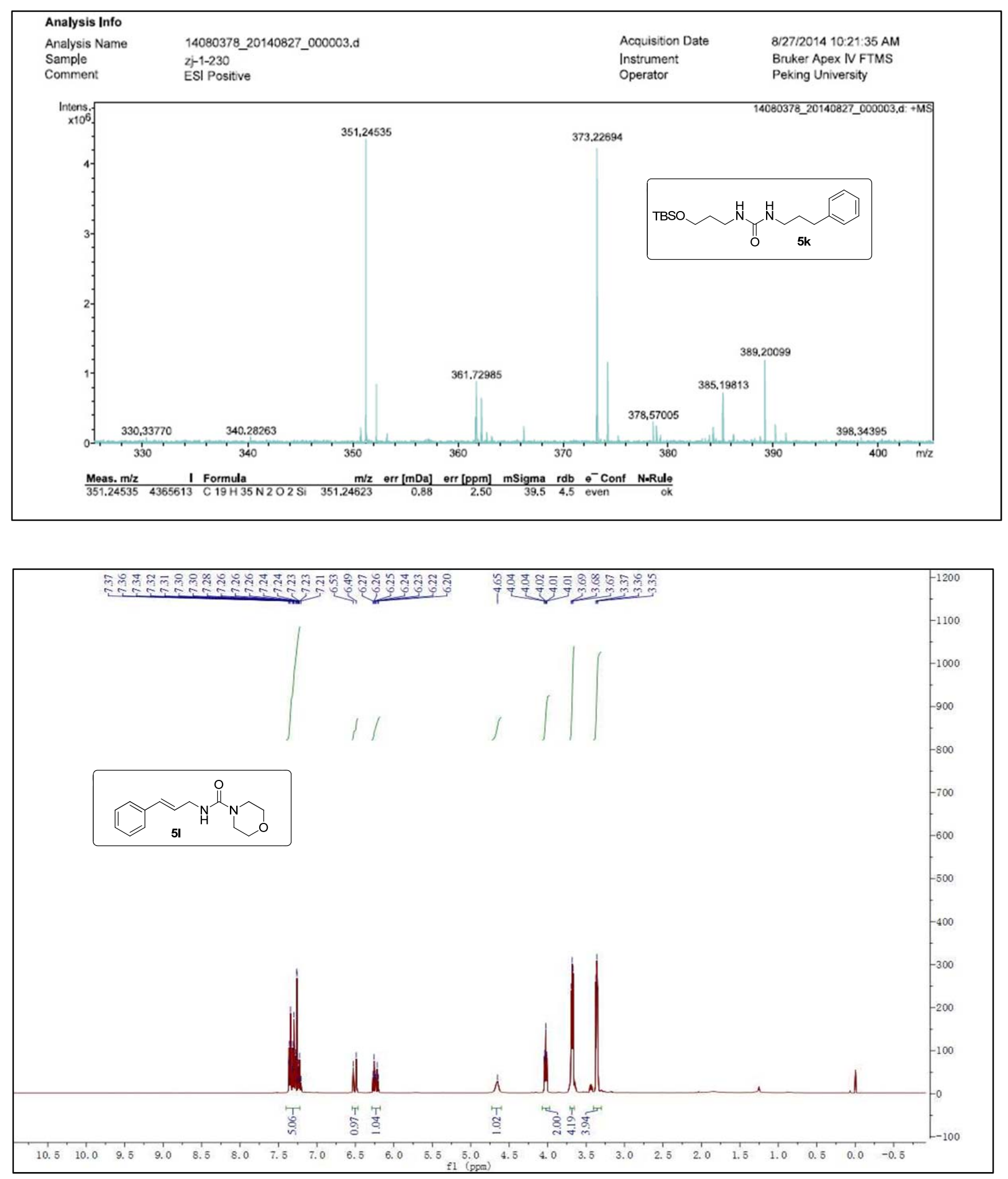

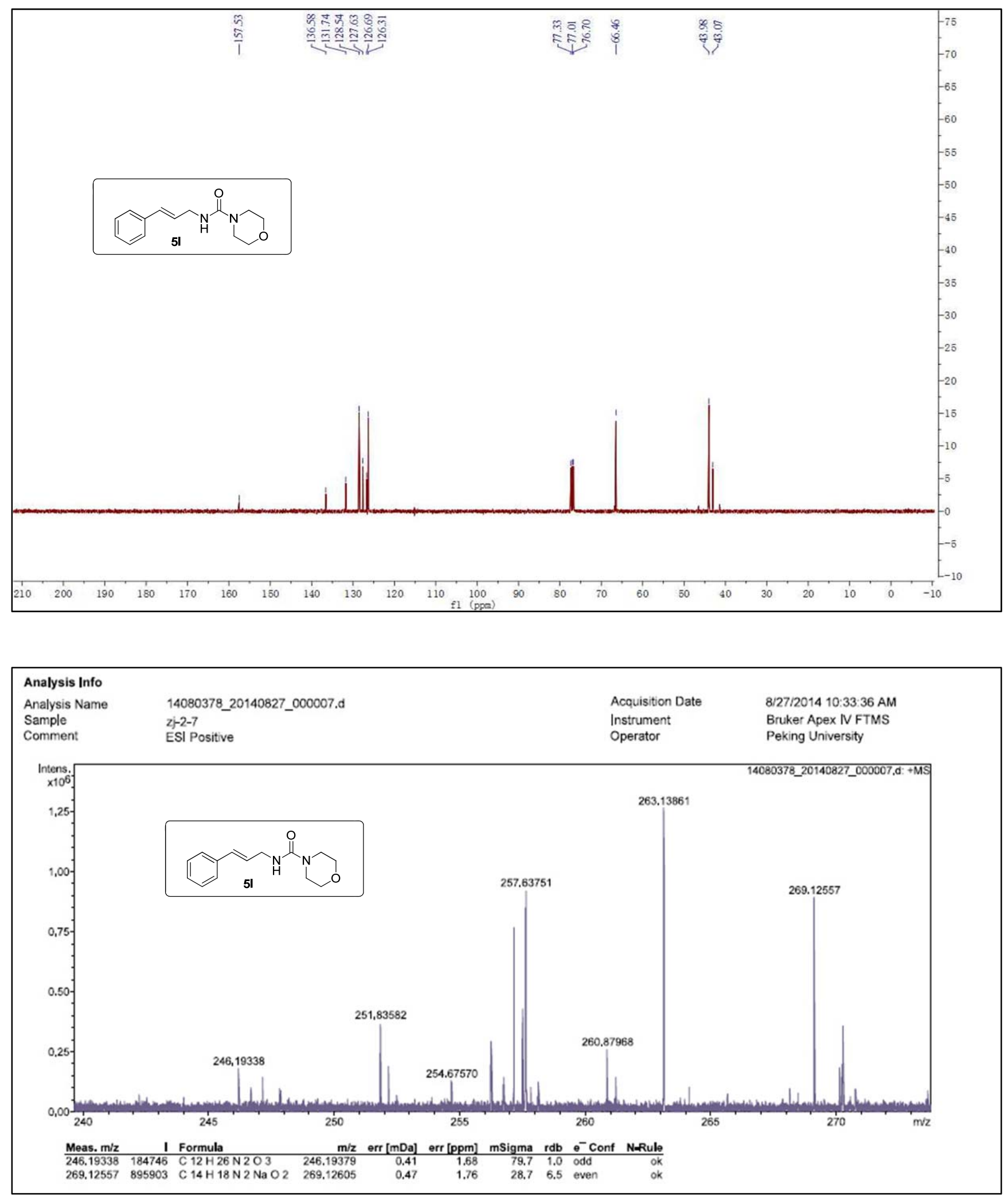

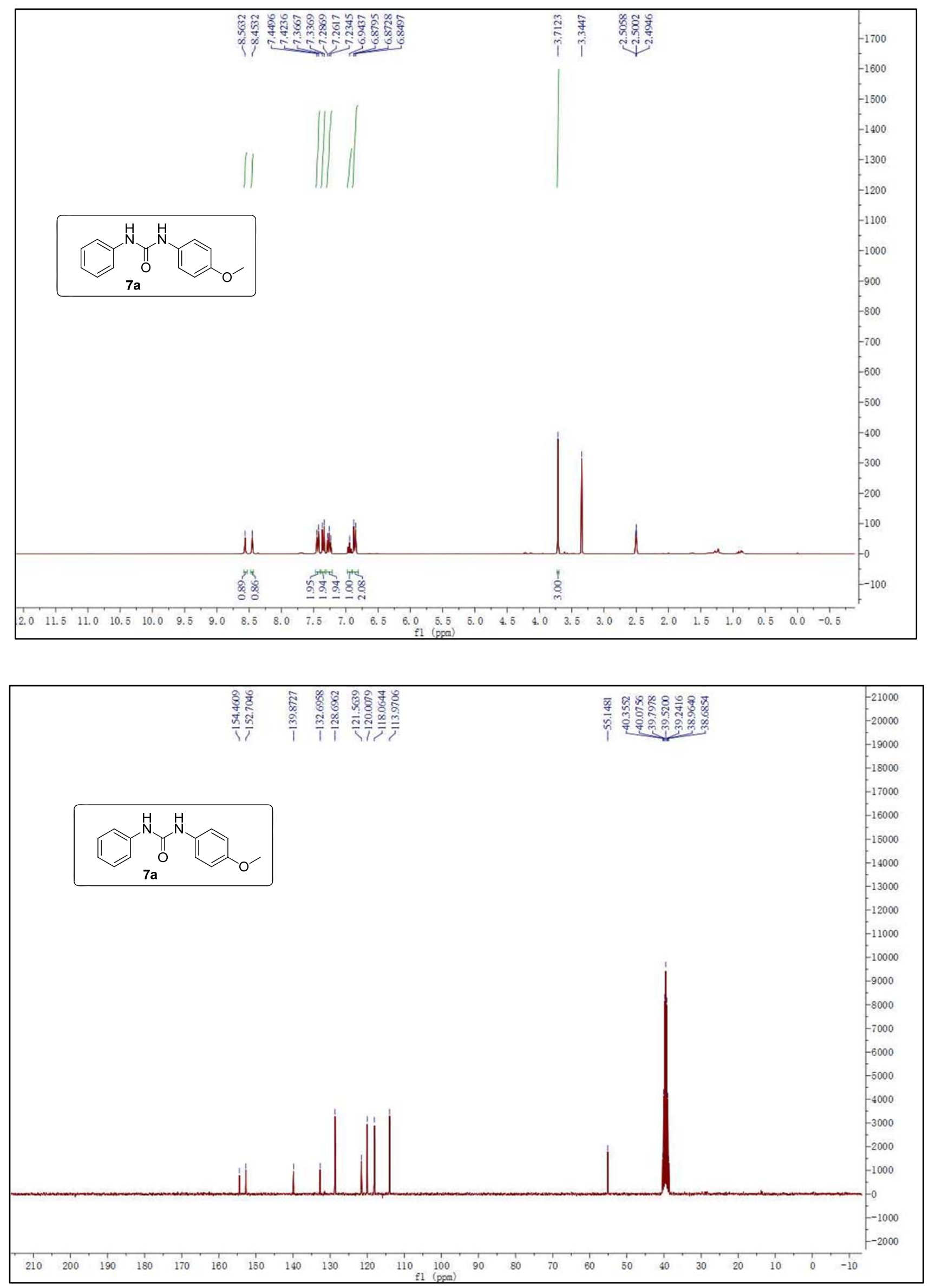

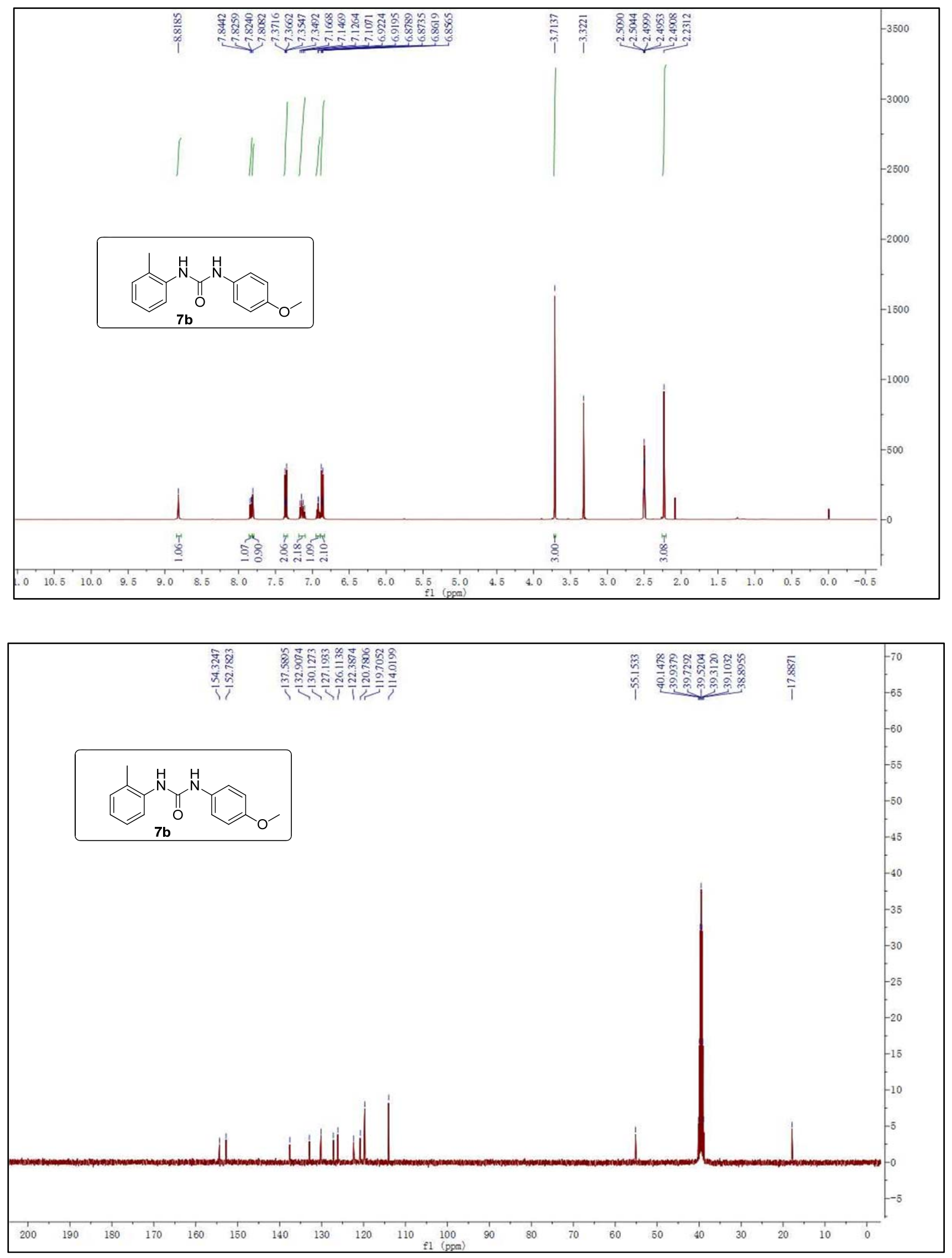

66 

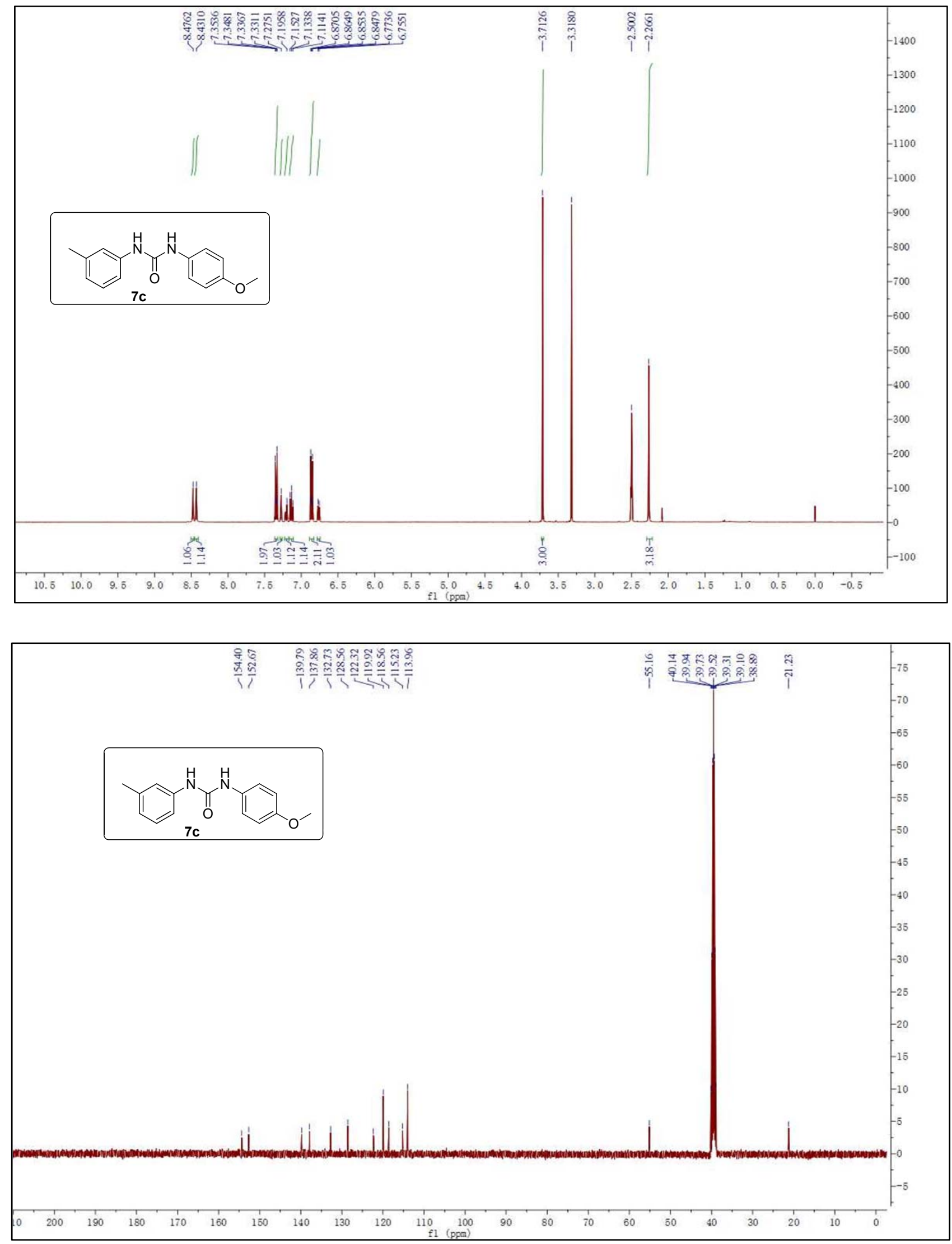

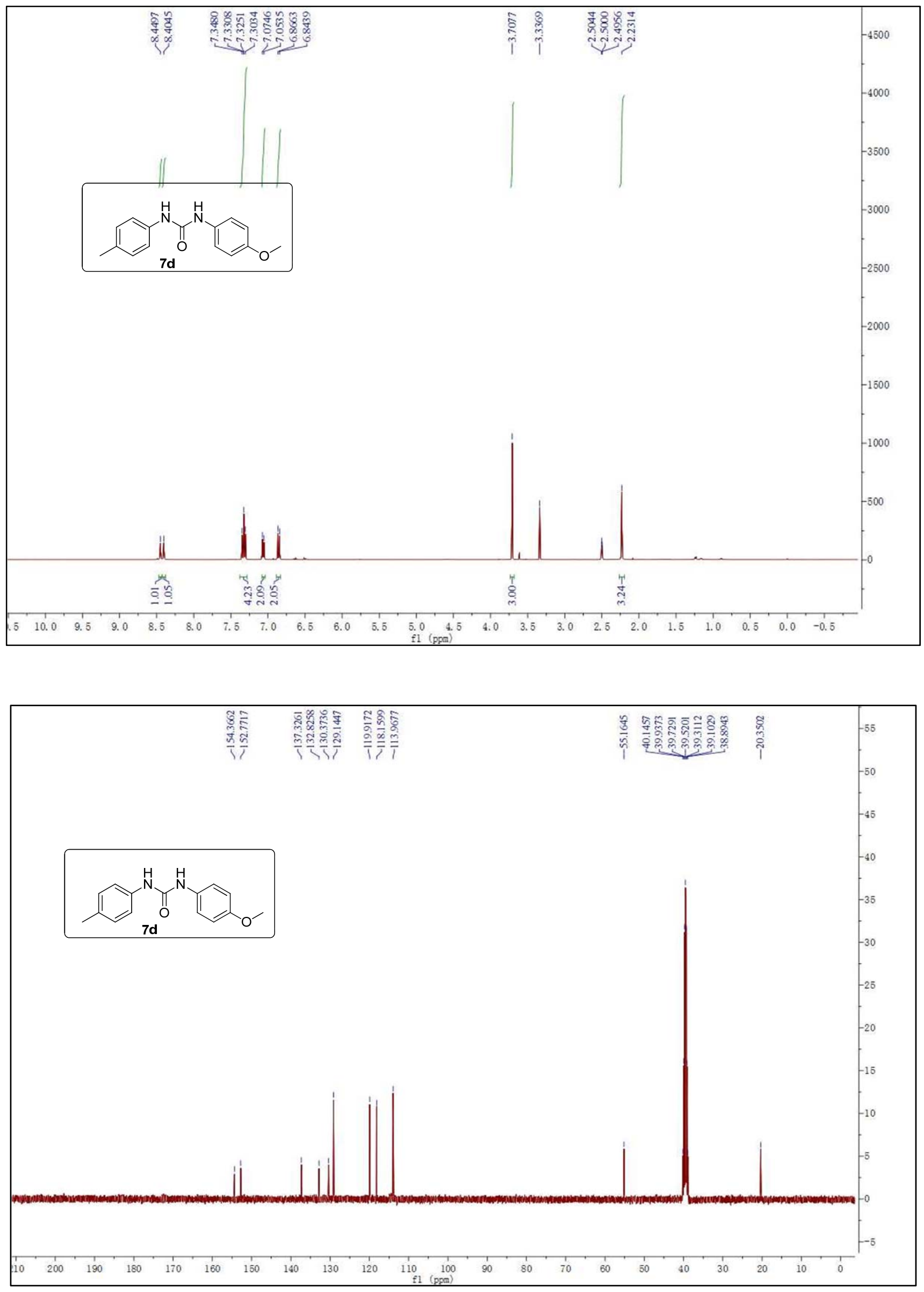

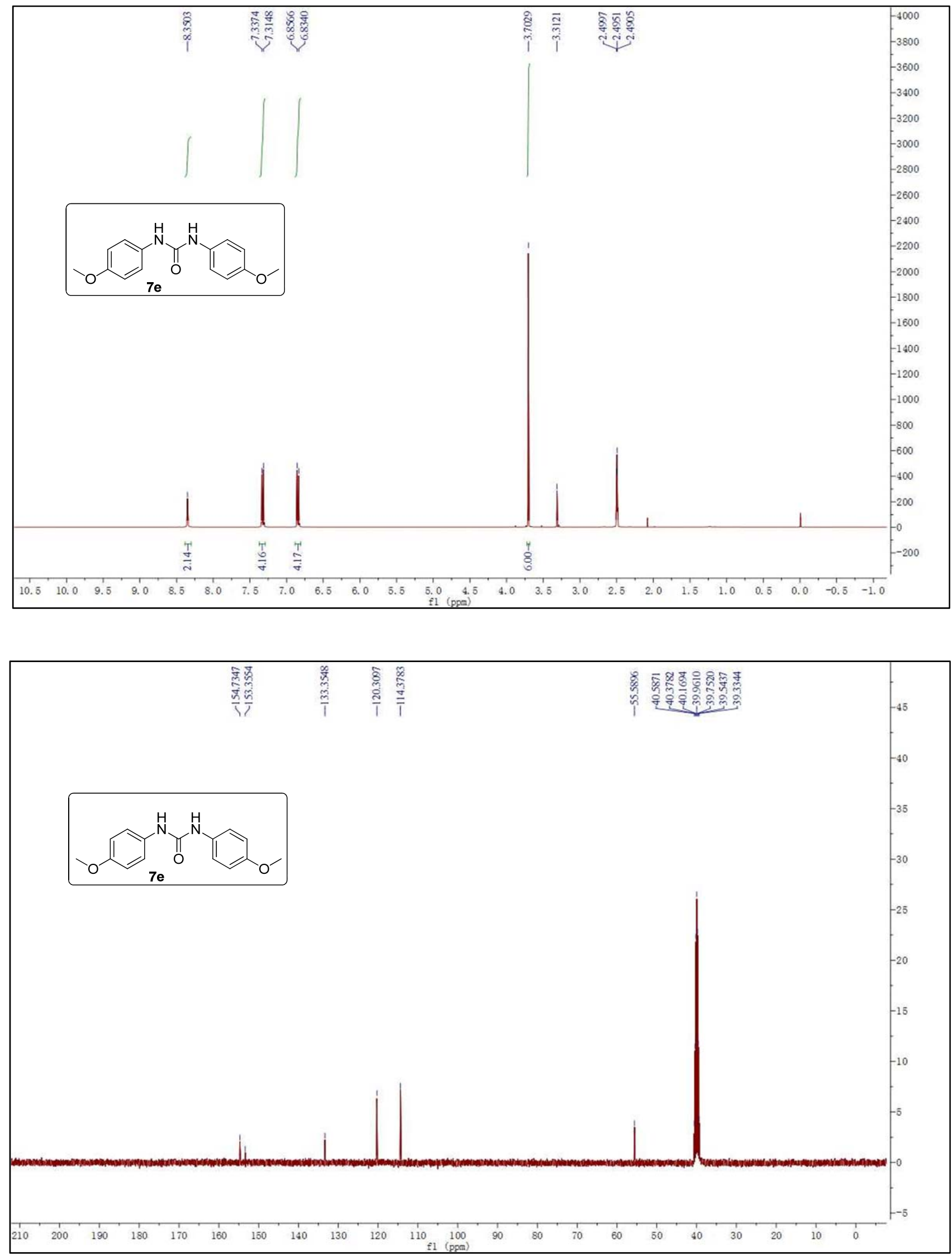

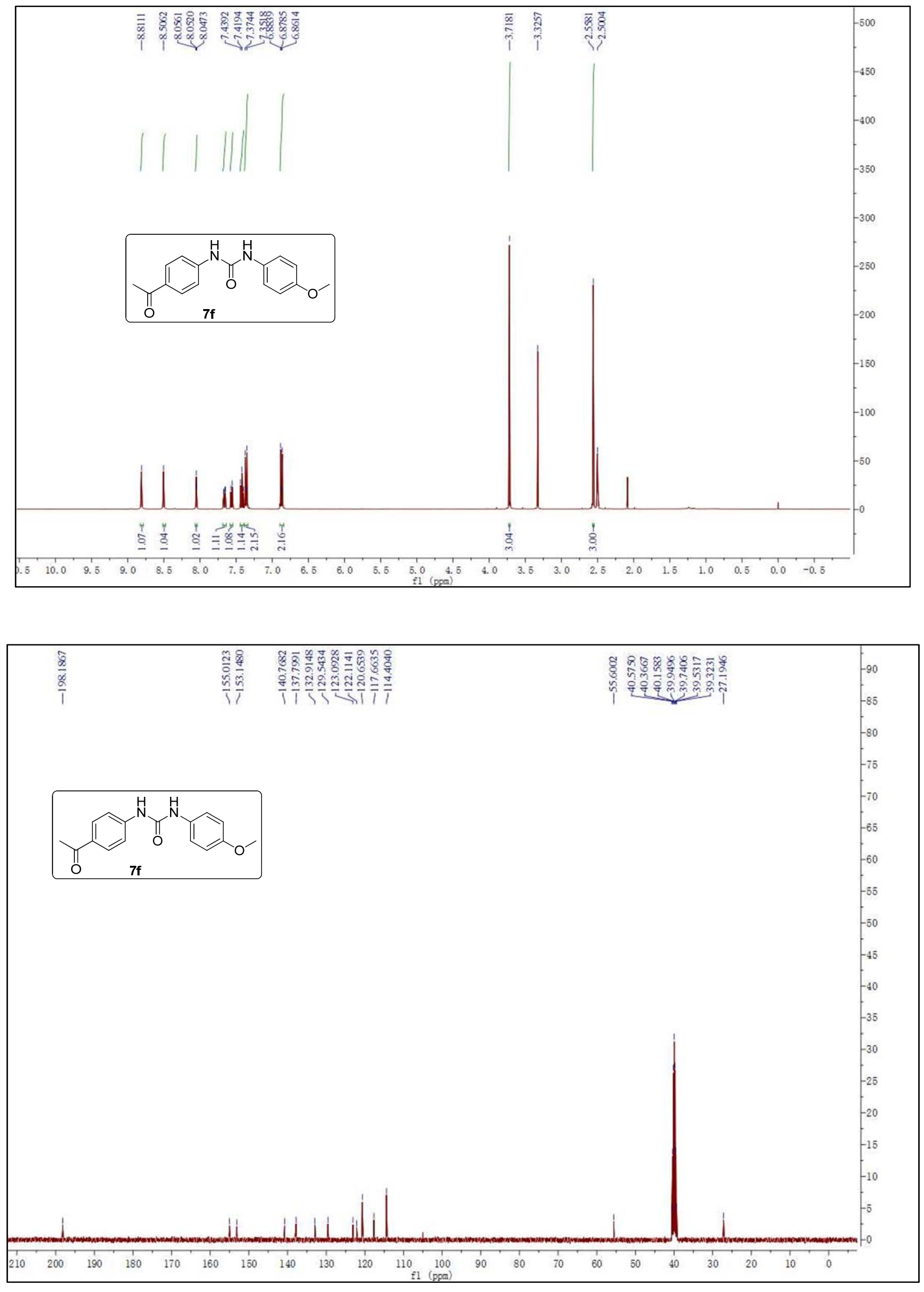

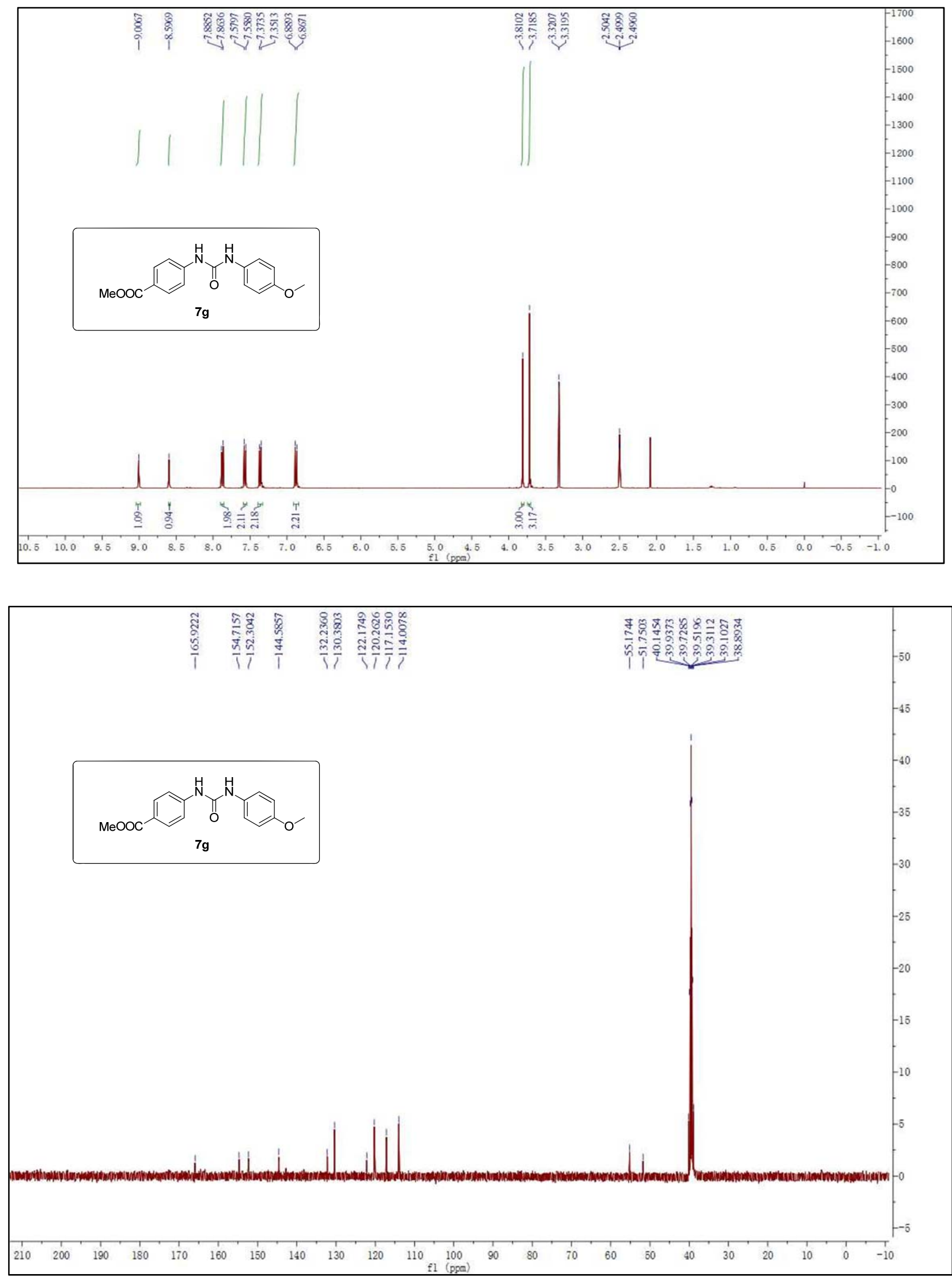

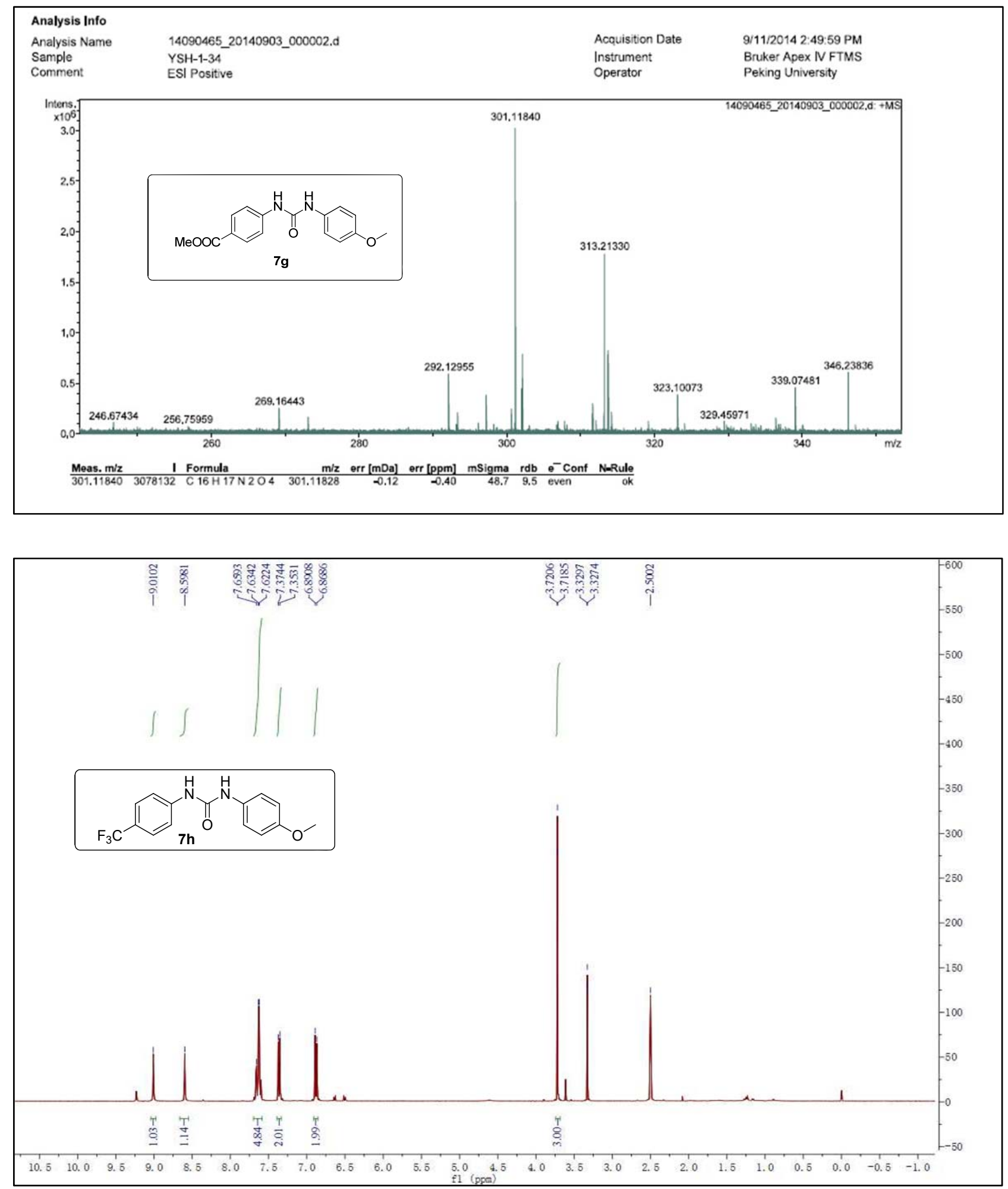

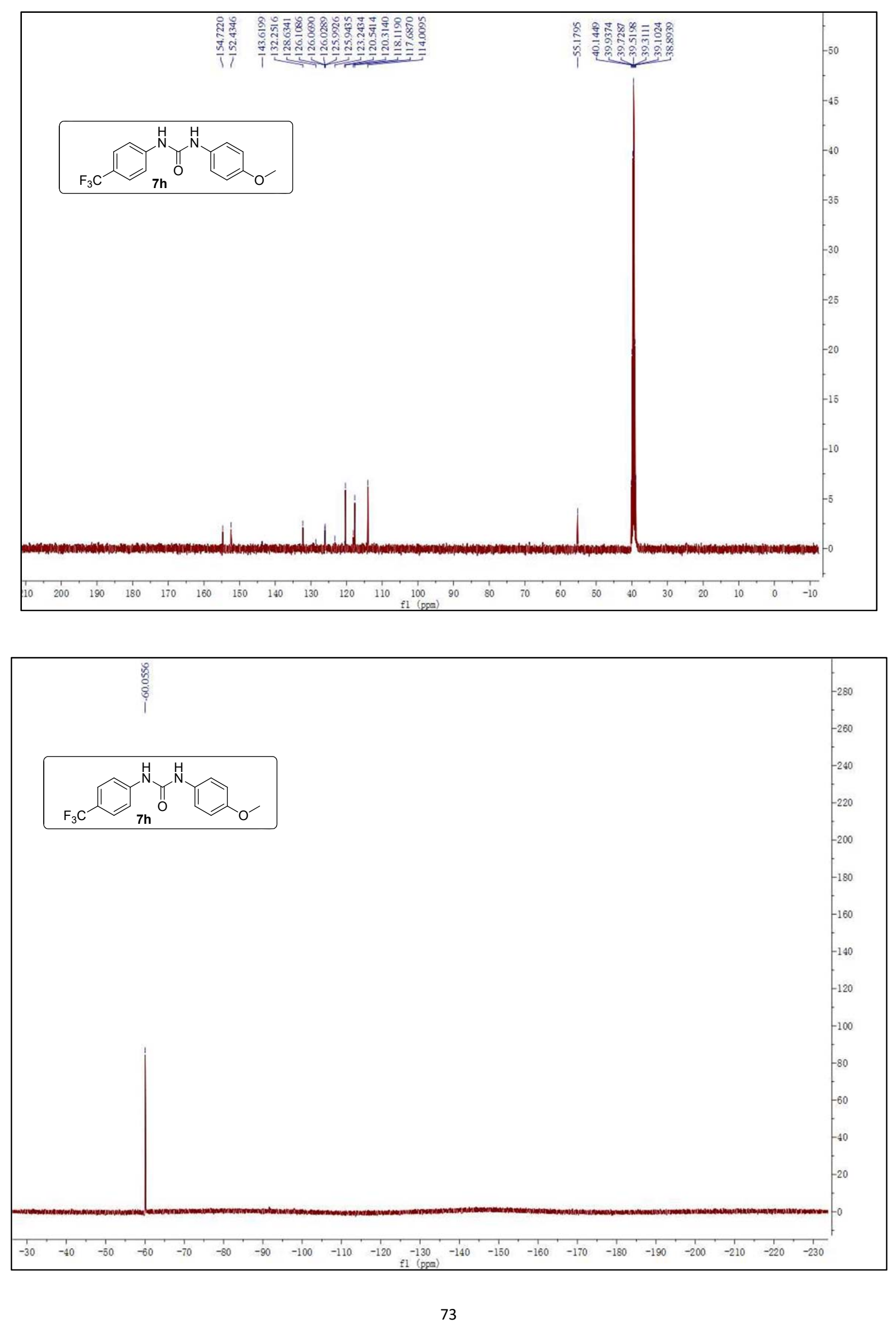

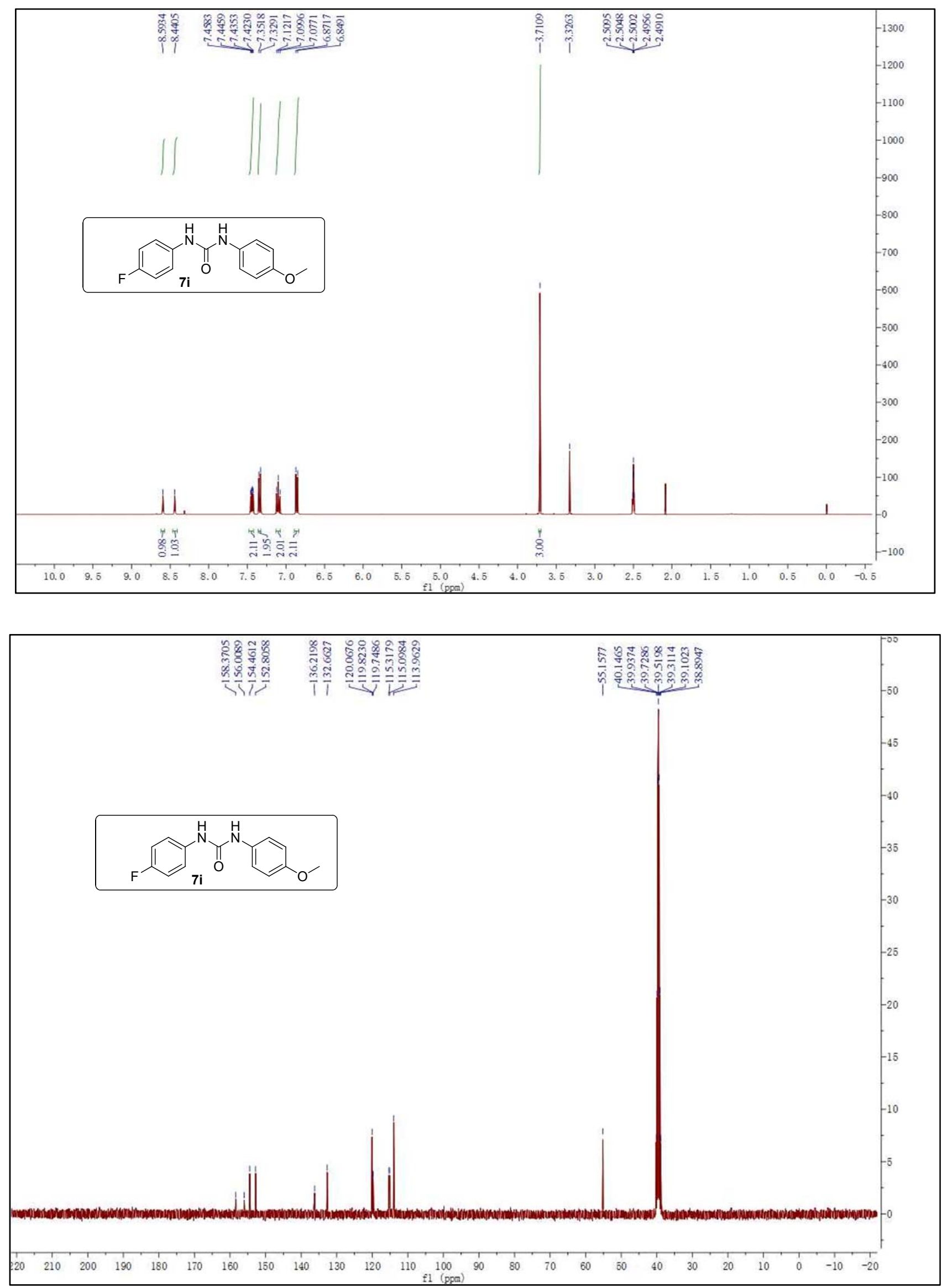

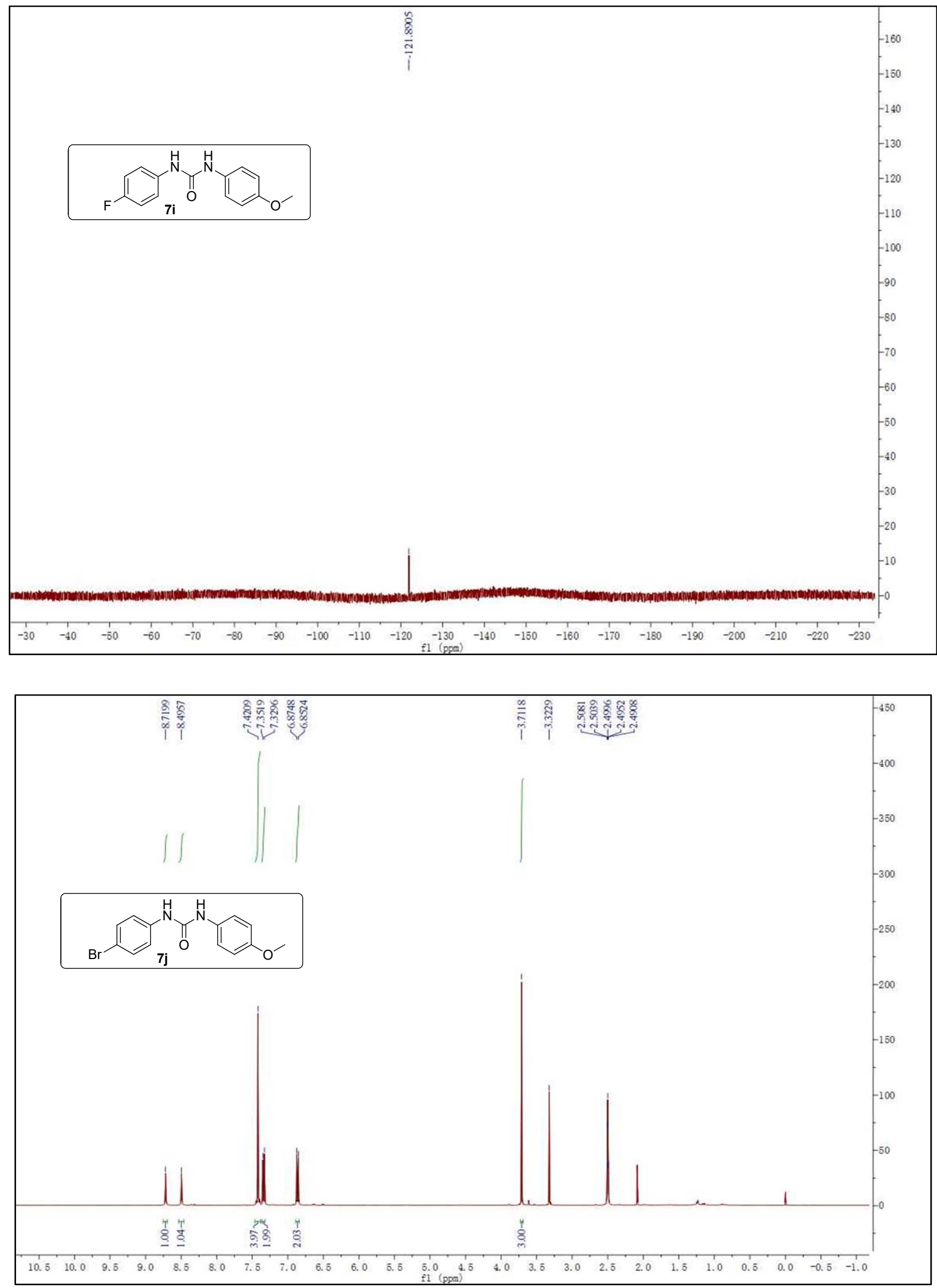

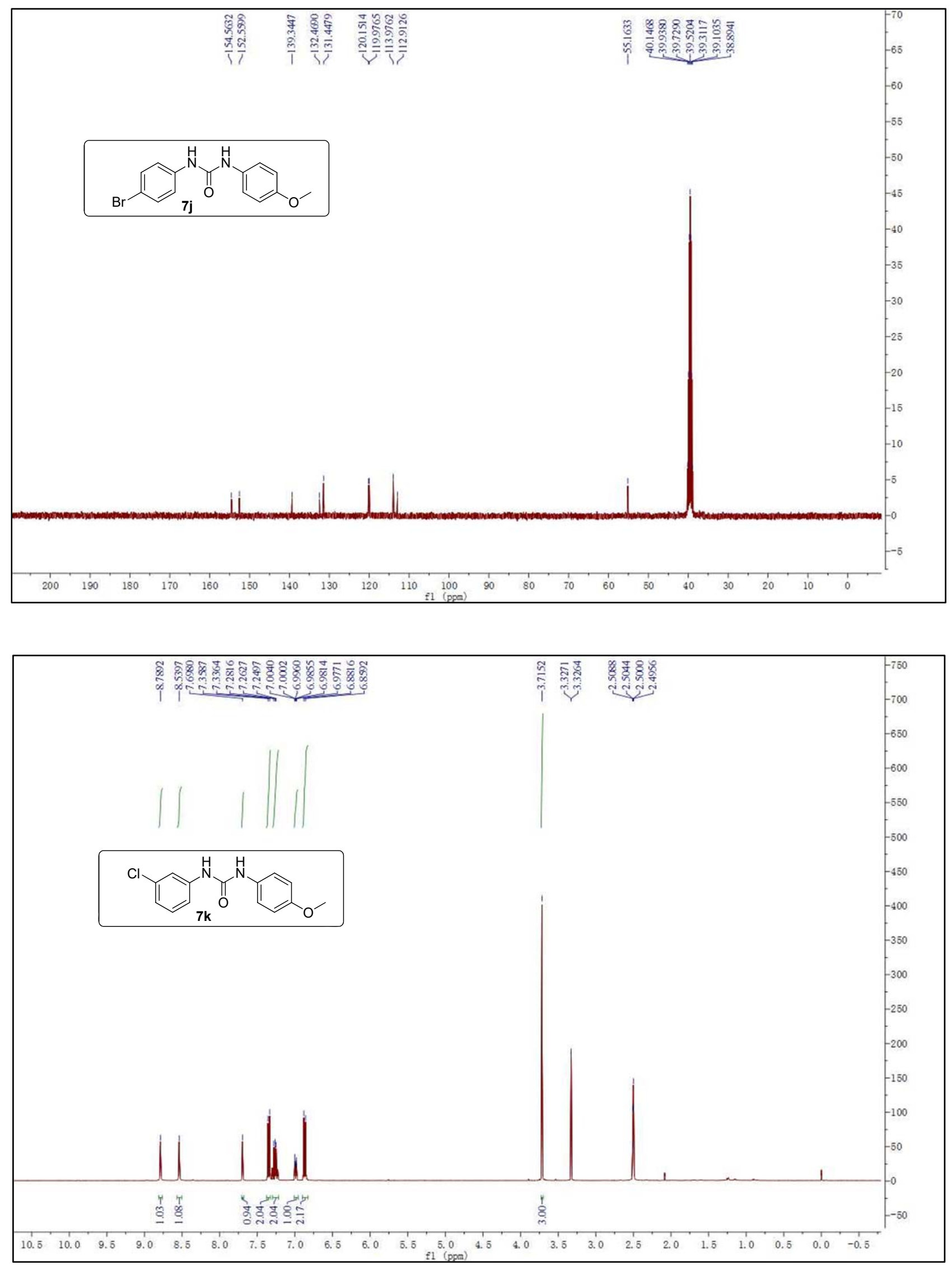

76 

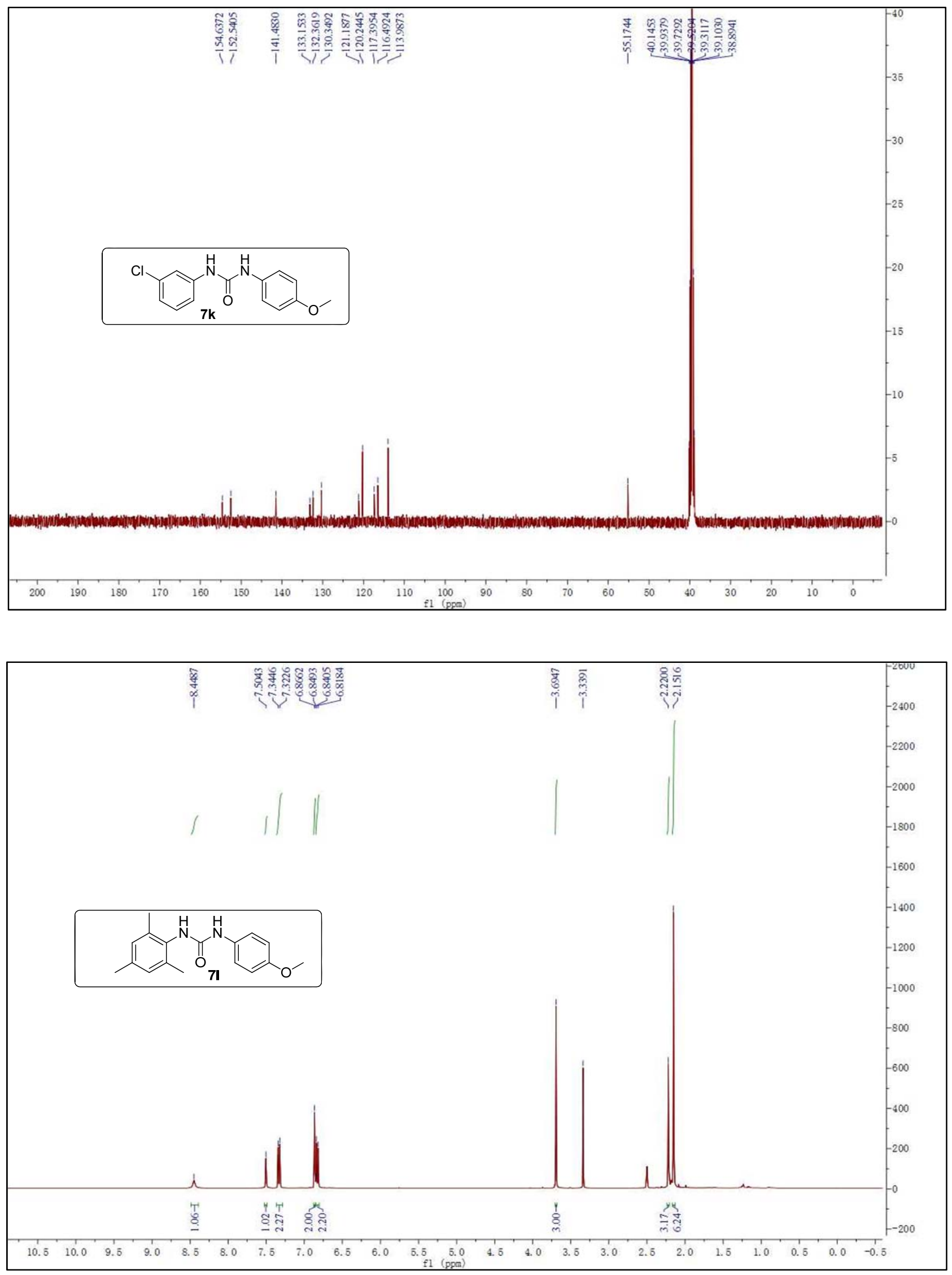

77 

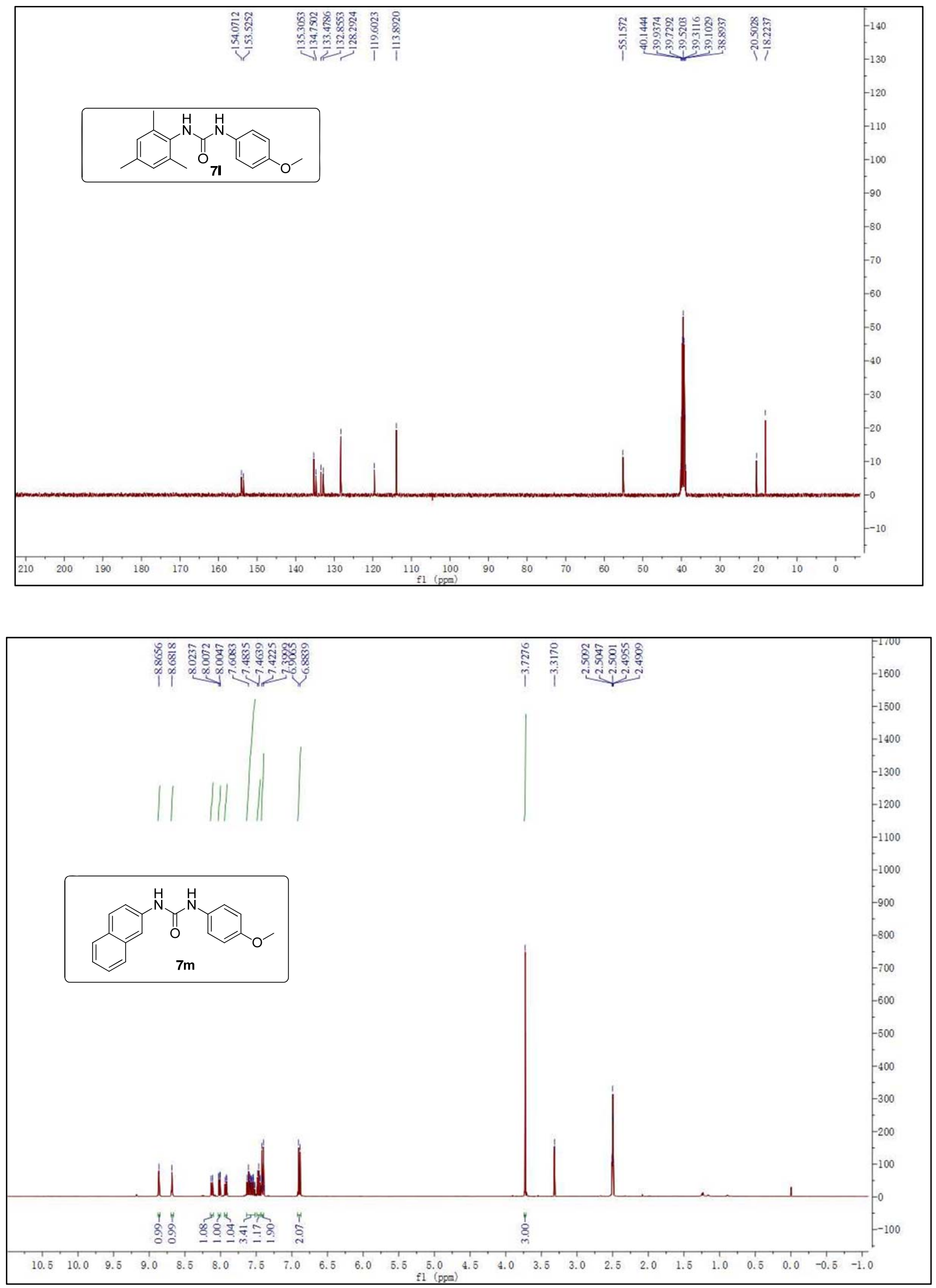

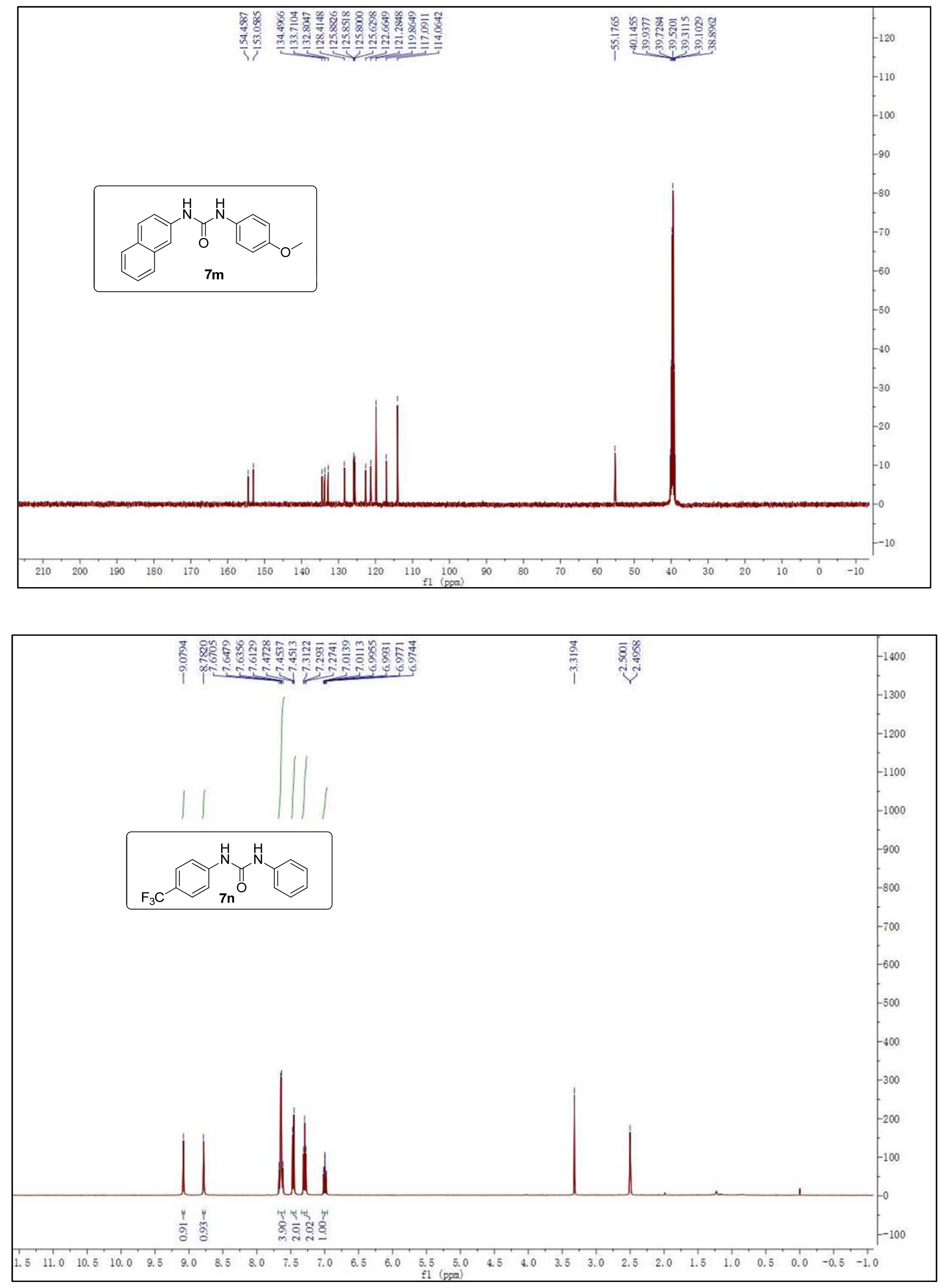

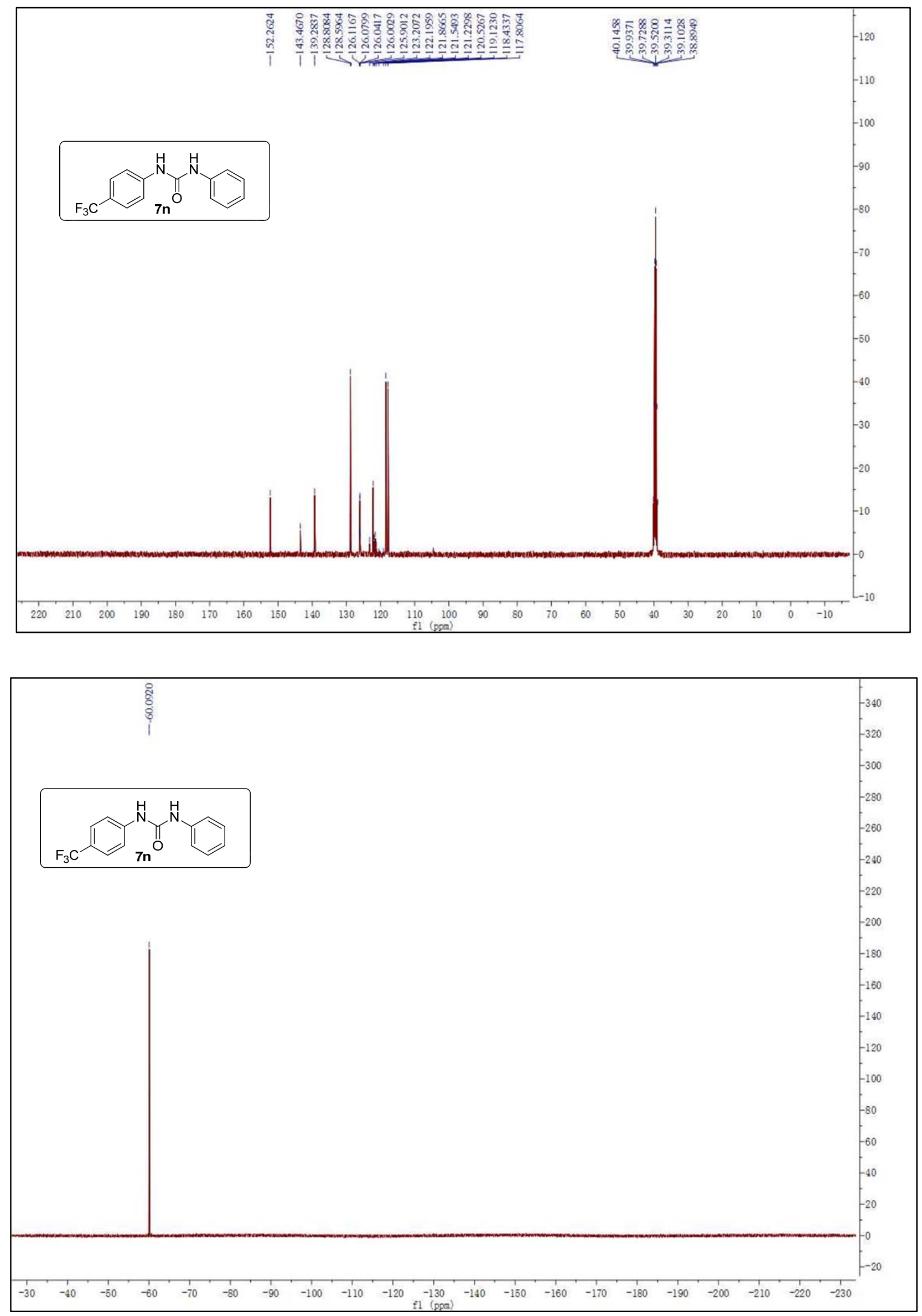

80 

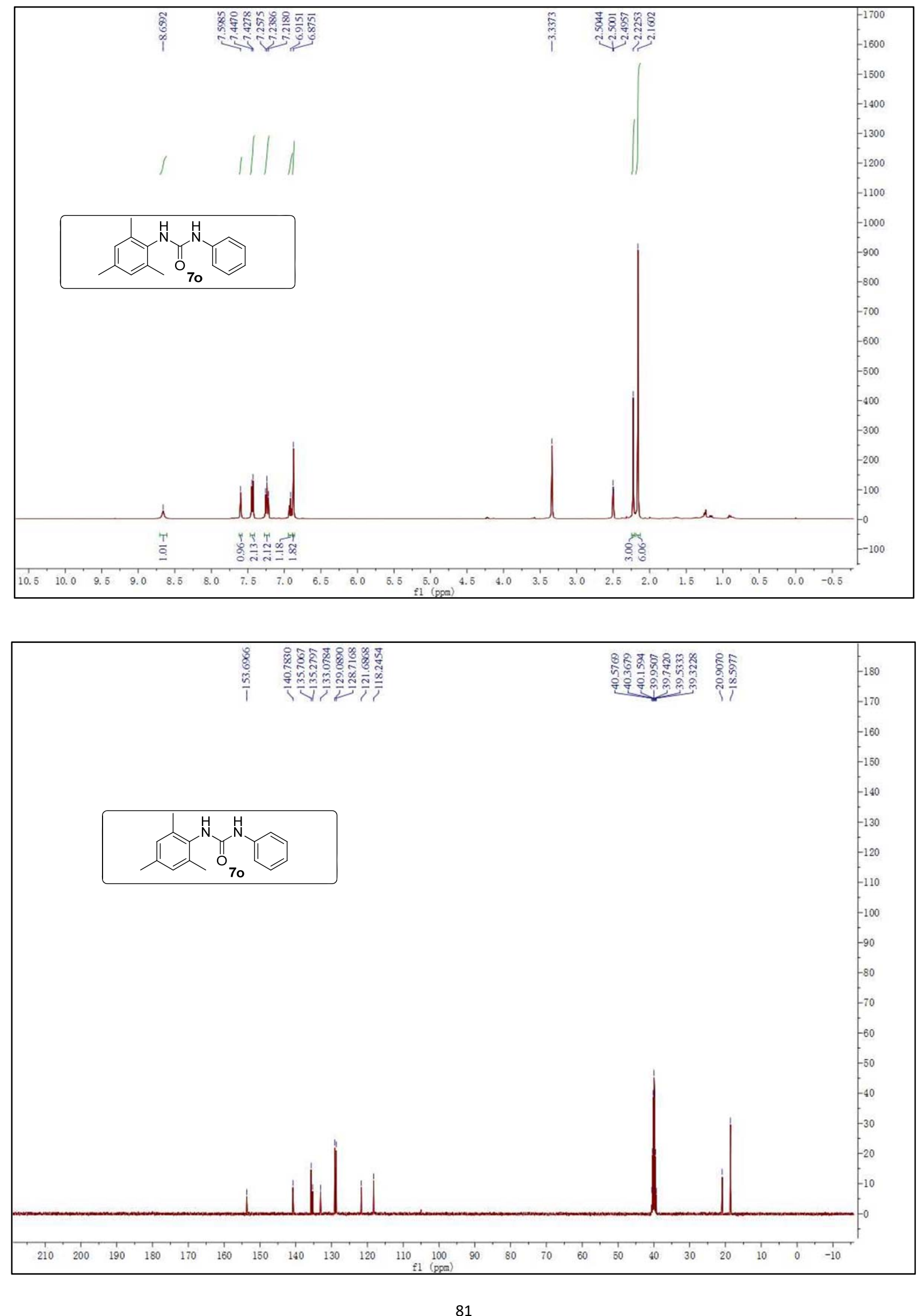

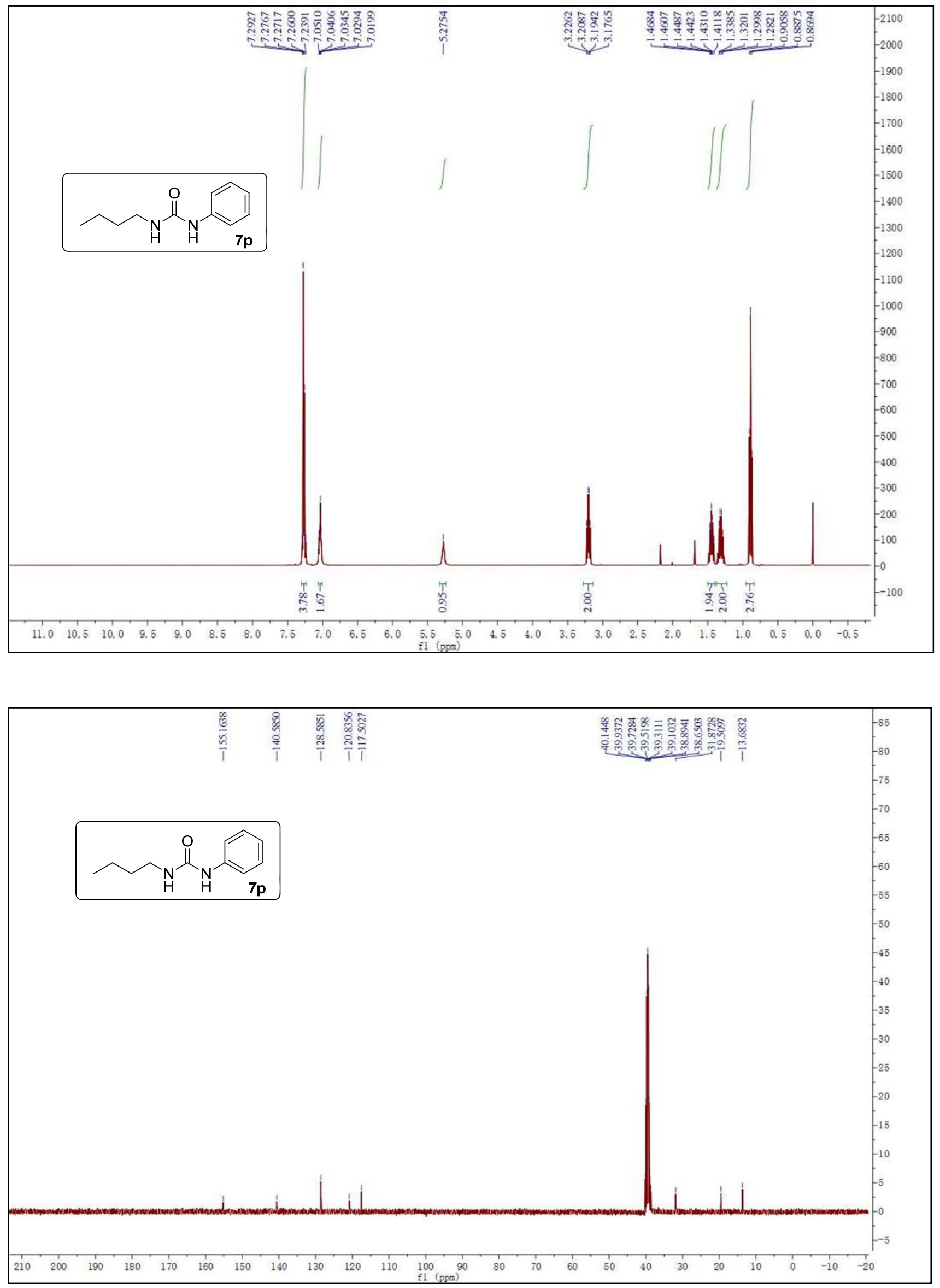

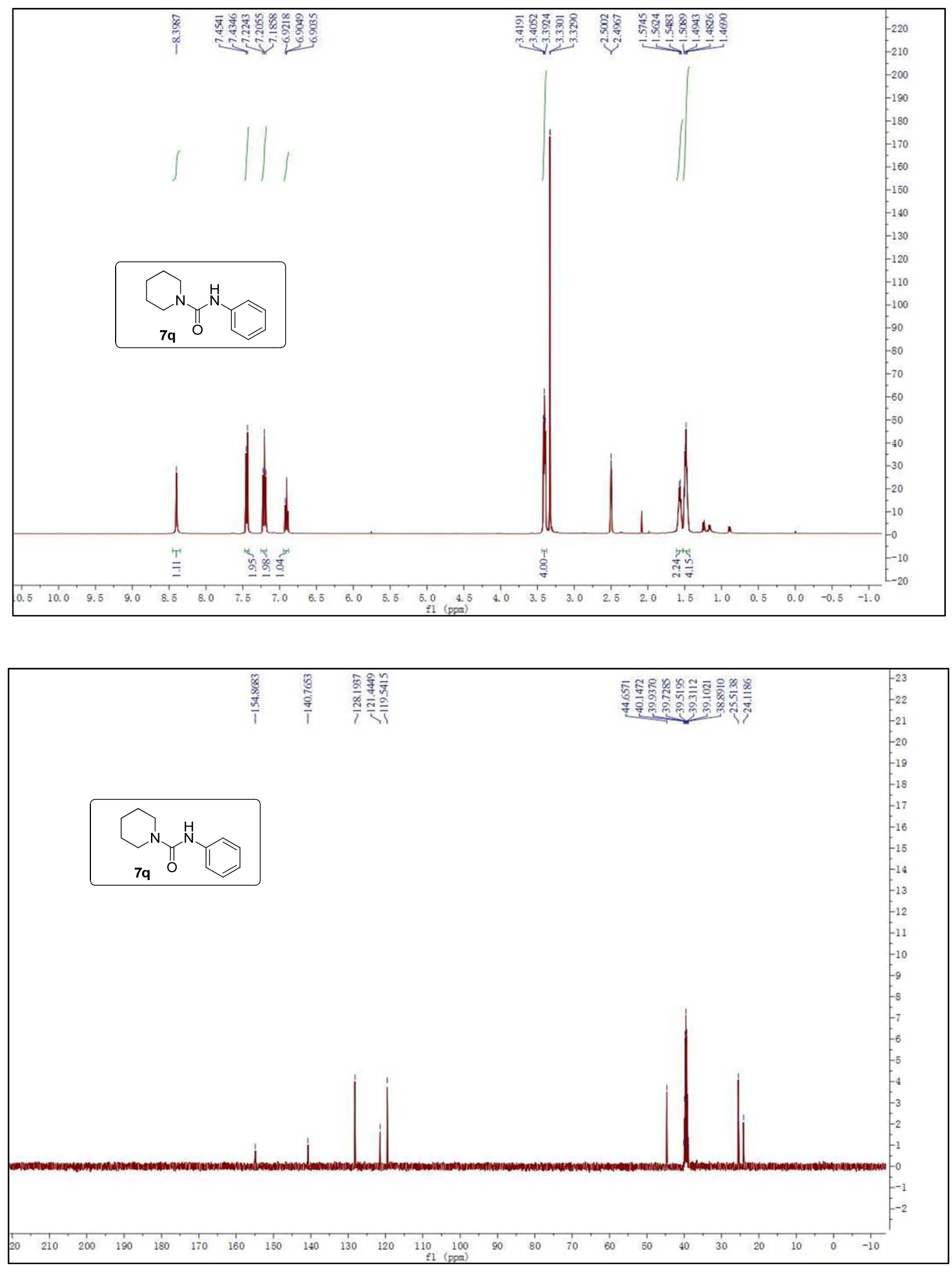

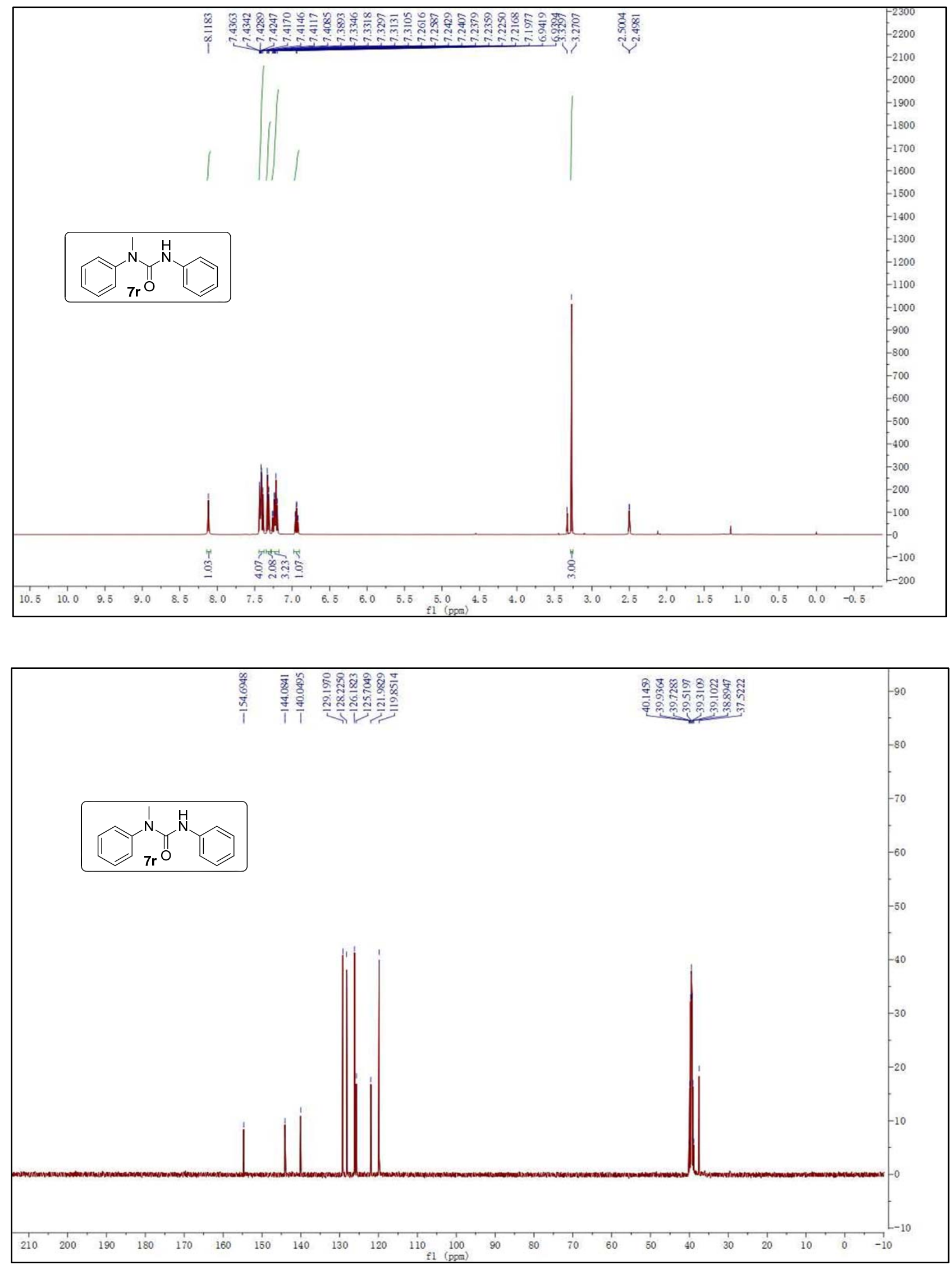